\title{
Sutures and contact homology I
}

\author{
VINCENT COLIN \\ PAOLO GHIGGini \\ KO HONDA \\ MichaEL HutCHINGS
}

\begin{abstract}
We define a relative version of contact homology for contact manifolds with convex boundary and prove basic properties of this relative contact homology. Similar considerations also hold for embedded contact homology.
\end{abstract}

57M50; 53D10, 53D40

\section{Introduction and main results}

The goal of this paper is to define relative versions of contact homology and embedded contact homology for contact manifolds with convex boundary and to prove basic properties of these relative contact homology theories. Contact homology, due to Eliashberg and Hofer and part of the symplectic field theory (SFT) package of Eliashberg, Givental and Hofer [11], is a Floer-type invariant of a (closed) contact manifold. It is the homology of a differential graded algebra whose differential counts genus zero holomorphic curves in the symplectization with one positive puncture and an arbitrary number of negative punctures. Contact homology has been quite successful at distinguishing contact structures, as can be seen for example from the works of Bourgeois and Colin [1] and Ustilovsky [38]. Embedded contact homology (ECH) is a variant of contact homology/SFT for three-dimensional contact manifolds, defined by Hutchings [24] with Sullivan [26] and Taubes [28; 29], which is the homology of a chain complex whose differential counts certain embedded holomorphic curves, possibly of higher genus, in the symplectization. Although ECH is defined in terms of a contact form, it is actually a topological invariant of the underlying 3-manifold, ie it does not depend on the contact structure (up to a possible grading shift - see Section 1.1). This invariance follows from a theorem of Taubes [37] identifying ECH with Seiberg-Witten Floer cohomology, which also implies the Weinstein conjecture in dimension three, again by work of Taubes [36].

Let $M$ be a compact, oriented $(2 n+1)$-dimensional manifold with boundary. A natural boundary condition for an oriented contact structure $\xi$ on $M$ to satisfy is that $\partial M$ be 
$\xi$-convex. The notion of convexity in contact geometry was introduced by Eliashberg and Gromov [12], and developed by Giroux [15]. A thorough discussion will be given in Section 2.2, but we briefly give definitions here: A $2 n$-dimensional submanifold $\Sigma \subset M$ is $\xi$-convex if there is a contact vector field $X$ transverse to $\Sigma$. To a $\xi$-convex submanifold $\Sigma$ and a transverse contact vector field $X$ we can associate the dividing set $\Gamma=\Gamma_{X} \subset \Sigma$, namely the set of points $x \in \Sigma$ such that $X(x) \in \xi(x)$. By the contact condition, $(\Gamma, \xi \cap T \Gamma)$ is a $(2 n-1)$-dimensional contact submanifold of $(M, \xi)$; the isotopy class of $(\Gamma, \xi \cap T \Gamma)$ is independent of the choice of $X$. The set of points $x \in \Sigma$ where $X$ is positively (resp. negatively) transverse to $\xi$ will be denoted by $R_{+}(\Gamma)$ (resp. $R_{-}(\Gamma)$ ). We denote by $(M, \Gamma, \xi)$ the contact manifold $(M, \xi)$ with convex boundary and dividing set $\Gamma=\Gamma_{X} \subset \partial M$ with respect to some transverse contact vector field $X$. We emphasize that, in this paper, $\Gamma$ is a submanifold of $\Sigma$, not an isotopy class of submanifolds of $\Sigma$.

\subsection{Invariants of sutured contact manifolds}

1.1.1 Sutured contact homology and sutured ECH Our first result is that the contact homology algebra and, in the three-dimensional case, embedded contact homology can be defined for a contact manifold $(M, \Gamma, \xi)$ with convex boundary, extending the usual definitions. A slight subtlety is that the actual boundary condition we want to use is not that $\partial M$ be $\xi$-convex, but rather that $(M, \Gamma, \xi)$ be a sutured contact manifold. Roughly speaking this is a sutured manifold, essentially as defined by Gabai [14], with a contact structure adapted to the sutures. The precise definition of sutured contact manifold is given in Section 2.3, and Section 4.1 explains how to pass between the convex and sutured boundary conditions. For now we write $(M, \Gamma, \xi)$ to indicate either of these boundary conditions, and we refer to $\Gamma$ interchangeably as a "suture" or a "dividing set".

Theorem 1.1 Let $(M, \Gamma, \xi)$ be a $(2 n+1)$-dimensional sutured contact manifold.

(1) The contact homology algebra $H C(M, \Gamma, \xi)$ is defined and independent of the choice of contact $1-$ form $\alpha$ with $\operatorname{ker} \alpha=\xi$, adapted almost complex structure $J$, and abstract perturbations.

(2) Suppose $\operatorname{dim} M=3$. Then the embedded contact homology $\operatorname{ECH}(M, \Gamma, \alpha, J)$ is defined.

Here contact homology is defined over $\mathbb{Q}$. One reason for this is that multiply covered Reeb orbits force one to use coefficients in $\mathbb{Q}$ or some extension thereof. On the other hand, ECH is defined over $\mathbb{Z}$. 
The definitions of these versions of contact homology, as well as the proof of Theorem 1.1, are given in Section 6. The basic idea is to copy the definitions from the closed case, and to argue that the relevant Gromov compactness carries over.

Note that, already in the closed case, the definition and proof of invariance of contact homology require some abstract perturbations of the moduli spaces of holomorphic curves (due to the presence of multiply covered holomorphic curves of negative index). This construction is still in progress, using the polyfold technology being developed by Hofer, Wysocki and Zehnder; see Hofer [20]. The proof of Theorem 1.1(1) assumes that the machinery needed to construct contact homology in the closed case works; see Section 6 for details.

The differentials in both contact homology and ECH depend also on the choice of a coherent orientation of the moduli spaces; see Bourgeois and Mohnke [4] for contact homology and Hutchings and Taubes [29, Section 9] for ECH. Since the construction of the coherent orientation is local, it carries over unchanged in the sutured case. Different choices of coherent orientations yield different, but canonically isomorphic, chain complexes.

If $A$ is a homology class in $H_{1}(M)$, then we write $H C(M, \Gamma, \xi, A)$ for the homology of the subcomplex generated by monomials $\gamma_{1} \ldots \gamma_{k}$, where $\gamma_{i}$ is a closed orbit of the Reeb vector field $R_{\alpha}$ corresponding to $\alpha$, and $\sum_{i=1}^{k}\left[\gamma_{i}\right]=A$. Also write $E C H(M, \Gamma, \alpha, J, A)$ for the homology of the subcomplex generated by orbit sets $\left\{\left(\gamma_{i}, m_{i}\right)\right\}_{i=1}^{k}$ where $\sum_{i=1}^{k} m_{i}\left[\gamma_{i}\right]=A$.

\subsubsection{Conjectural topological invariance of sutured ECH In the closed case, ECH} is a topological invariant of the underlying 3-manifold in the following sense: If $M$ is a closed 3-manifold, if $\alpha_{i}$ is a contact form on $M$ and $J_{i}$ is a generic $\alpha_{i}$-adapted almost complex structure as needed to define the ECH chain complex for $i=1,2$, and if $A_{1} \in H_{1}(M)$, then

$$
\operatorname{ECH}\left(M, \alpha_{1}, J_{1}, A_{1}\right) \simeq \operatorname{ECH}\left(M, \alpha_{2}, J_{2}, A_{2}\right)
$$

as relatively graded $\mathbb{Z}$-modules, where

$$
A_{2}-A_{1}=\operatorname{PD}\left(\mathfrak{s} \xi_{1}-\mathfrak{s} \xi_{2}\right)
$$

Here $\mathfrak{s}_{i}$ denotes the $\operatorname{Spin}^{c}$ structure determined by $\xi_{i}=\operatorname{Ker}\left(\alpha_{i}\right)$, and $\mathfrak{s}_{\xi_{1}}-\mathfrak{s} \xi_{2} \in$ $H^{2}(M ; \mathbb{Z})$ denotes the difference between the two $\operatorname{Spin}^{c}$ structures. The above invariance follows from the theorem of Taubes [37] identifying $E C H_{*}\left(M, \alpha_{i}, J_{i}, A_{i}\right)$ 
with the Seiberg-Witten Floer cohomology $\widehat{H M}^{-*}\left(M, \mathfrak{s} \xi_{i}+\operatorname{PD}\left(A_{i}\right)\right)$, up to a possible grading shift ${ }^{1}$.

This motivates the following conjecture in the sutured case:

Conjecture 1.2 The sutured embedded contact homology $\operatorname{ECH}(M, \Gamma, \alpha, J)$ does not depend on the choice of contact form $\alpha$, contact structure $\xi=\operatorname{ker} \alpha$ or almost complex structure $J$. More precisely,

$$
\operatorname{ECH}\left(M, \Gamma, \alpha_{1}, J_{1}, A_{1}\right) \simeq \operatorname{ECH}\left(M, \Gamma, \alpha_{2}, J_{2}, A_{2}\right)
$$

as relatively graded $\mathbb{Z}$-modules, when $A_{1}$ and $A_{2}$ are related by (1).

Remark 1.3 We need to explain why Equation (1) still makes sense in the sutured case. The difference between two $\operatorname{Spin}^{c}$-structures on $M$ is an element of $H^{2}(M ; \mathbb{Z})=$ $H_{1}(M, \partial M)$. However for a sutured manifold one has a fixed 2-plane field on $\partial M$ determined by the sutures, which determines a canonical $\operatorname{Spin}^{c}$-structure $\mathfrak{s}_{0}$ in a neighborhood of $\partial M$. A contact structure $\xi$ compatible with the sutures then determines a relative $\operatorname{Spin}^{c}$-structure relative to $\mathfrak{s}_{0}$, which means a $\operatorname{Spin}^{c}$-structure $\mathfrak{s}_{\xi}$ on $M$ together with an isomorphism of $\left.\mathfrak{s}_{\xi}\right|_{\partial M}$ with $\mathfrak{s}_{0}$. These relative $\operatorname{Spin}^{c}$ structures comprise an affine space over $H^{2}(M, \partial M ; \mathbb{Z})=H_{1}(M)$.

1.1.3 Invariants of Legendrian submanifolds Let $(M, \xi)$ be a closed $(2 n+1)-$ dimensional contact manifold. Then we can define an invariant $\operatorname{HC}(M, \xi, L)$ of a Legendrian submanifold $L$ in $(M, \xi)$ as follows: Let $N(L)$ be a Darboux-Weinstein neighborhood of $L$. Then $\partial(M-N(L))$ is a convex submanifold of $M$ with dividing set $\Gamma_{\partial(M-N(L))}$. We now define

$$
H C(M, \xi, L)=H C\left(M-N(L), \Gamma_{\partial(M-N(L))},\left.\xi\right|_{M-N(L)}\right) .
$$

Similarly, in dimension three, if $\xi=\operatorname{ker} \alpha$, then we can define

$$
\operatorname{ECH}(M, \alpha, J, L)=\operatorname{ECH}\left(M-N(L), \Gamma_{\partial(M-N(L))}, \alpha^{\prime}, J^{\prime}\right),
$$

where $\alpha^{\prime}, J^{\prime}$ are obtained from $\alpha, J$ by a modification near $\partial N(L)$. If Conjecture 1.2 is true, then $\operatorname{ECH}(M, \alpha, J, L)$ depends only on the ambient manifold $M$ and the framing of the knot $L$, as a relatively graded $\mathbb{Z}$-module. The details of the Legendrian submanifold invariants are given in Section 7.3.

${ }^{1}$ Both Seiberg-Witten Floer homology and ECH have absolute gradings by homotopy classes of oriented 2-plane fields on $M$ (see $[32 ; 25]$ ), and it is natural to conjecture that Taubes's isomorphism between them respects these gradings. 


\subsection{Comparison with sutured Floer homology}

In this section $\operatorname{dim} M=3$.

The definition of the sutured versions of contact homology theories has been known at least since the work of the first and third authors [8]. However, additional impetus for the current work came from the recent foundational work of Juhász [30; 31] on the sutured version of Heegaard Floer homology. Juhász' work also motivated the definition of a sutured version of Seiberg-Witten Floer homology by Kronheimer and Mrowka [33].

Definition 1.4 A sutured 3-manifold $(M, \Gamma)$ (see Section 2.3) is called balanced if $M$ has no closed components, the map $\pi_{0}(\Gamma) \rightarrow \pi_{0}(\partial M)$ is surjective, and $\chi\left(R_{+}(\Gamma)\right)=$ $\chi\left(R_{-}(\Gamma)\right)$ on the boundary of each component of $M$.

To a balanced sutured 3-manifold $(M, \Gamma)$, Juhász assigned the sutured Floer homology module $\operatorname{SFH}(M, \Gamma)$, which generalizes the "hat" version of Heegaard Floer homology and link Floer homology as follows. Let $M$ be a closed oriented 3-manifold. If we define the sutured manifold $M(1)$ to be the pair consisting of $M-B^{3}$ and suture $S^{1}$ on $\partial B^{3}$, then one has

$$
S F H(M(1)) \simeq \widehat{H F}(M),
$$

where the right hand side is the "hat" version of Heegaard Floer homology. Next, if $L \subset M$ is a link, define the sutured manifold $M(L)$ to be the pair consisting of $M-N(L)$ and suture which consists of two meridian curves on each component of $\partial N(L)$. Juhász then showed that $\operatorname{SFH}(M(L))$ is isomorphic to the link Floer homology of $L$.

If $(M, \Gamma, \xi)$ is a sutured contact 3-manifold with no closed components, then the sutured manifold $(M, \Gamma)$ is automatically balanced. To see this, recall the Euler class formula

$$
\langle e(\xi), \Sigma\rangle=\chi\left(R_{+}(\Gamma)\right)-\chi\left(R_{-}(\Gamma)\right)
$$

for a $\xi$-convex surface $\Sigma$ with dividing set $\Gamma$. Since the boundary $\Sigma$ of each component of $M$ is homologically trivial, the claim follows. (The $\pi_{0}$ surjectivity holds because each component of $\partial M \backslash \Gamma$ is an exact symplectic manifold; see Section 2.3.) Conversely, if $(M, \Gamma)$ is a balanced sutured 3-manifold, then there is a contact structure $\xi$ so that $\partial M$ is convex with dividing set $\Gamma$. (Moreover, according to Honda, Kazez and Matić [21], there is a tight (or universally tight) $\xi$ with convex boundary and dividing set $\Gamma$ on $\partial M$ if and only if $(M, \Gamma)$ is a taut sutured manifold, which means roughly that $R_{ \pm}(\Gamma)$ is incompressible and genus-minimizing in its homology class 
in $H_{2}(M, \Gamma)$.) In this paper we will assume without further mention that our sutured 3-manifolds are balanced.

If $M$ is closed, it is conjectured that $\mathrm{ECH}$ is isomorphic to Heegaard Floer homology, namely $\operatorname{ECH}(M, \xi, A) \simeq H F^{+}\left(-M, \mathfrak{s}_{\xi}+\mathrm{PD}(A)\right)$ as relatively graded $\mathbb{Z}$-modules. Extending this to the sutured case, we conjecture the following, which is a strengthening of Conjecture 1.2:

Conjecture 1.5 If $(M, \Gamma, \xi)$ is a sutured contact 3-manifold, then

$$
\operatorname{ECH}(M, \Gamma, \xi, A) \simeq \operatorname{SFH}(-M,-\Gamma, \mathfrak{s} \xi+\operatorname{PD}(A))
$$

as relatively graded $\mathbb{Z}$-modules, where $\mathfrak{s}_{\xi}$ denotes the relative $\operatorname{Spin}^{c}$-structure determined by $\xi$.

Calculations due to Golovko [18; 17] confirm this conjecture in some examples, eg, when $M=S^{1} \times D^{2}$ and $\Gamma$ is arbitrary, for a universally tight contact structure.

In the closed case, it is further conjectured that the isomorphism between $E C H$ and $\mathrm{HF}^{+}$intertwines the $U$-maps on both sides. Assuming this conjecture, we can confirm Conjecture 1.5 for the sutured contact 3-manifold $M(1)$, where $M$ is closed, as follows. On the Heegaard Floer side, the map $U: H^{+}(M) \rightarrow H F^{+}(M)$ fits into an exact triangle with $\widehat{H F}(M)$ in the third position. To obtain an analogue of this on the ECH side, define $\widehat{E C H}(M)$ to be the homology of the mapping cone of the $U$-map on the ECH chain complex. We then have the following analogue of (2):

Theorem 1.6 If $M$ is a closed oriented 3-manifold, then $\operatorname{ECH}(M(1))$ is independent of choices (as a relatively graded $\mathbb{Z}-$ module), and

$$
E C H(M(1)) \simeq \widehat{E C H}(M) .
$$

Arguments in Section 8.4 show that $E C H(M(1))$ depends only on the contact structure. The rest of Theorem 1.6 will be proved in the sequel [7].

We also have some evidence for Conjecture 1.5 for the sutured manifold $M(K)$, where $K$ is a nullhomologous knot in a closed oriented 3-manifold $M$. Namely, in Section 7.2 we define a filtration on the chain complex whose homology gives $E C H(M(1))$; the associated graded complex gives $E C H(M(K))$. This is analogous to the Heegaard Floer story, where there is a filtration on the chain complex computing $\widehat{H F}(M) \simeq S F H(M(1))$, such that the homology of the associated graded complex is the knot Floer homology, which is identified with $\operatorname{SFH}(M(K))$. 


\subsection{The simplest sutured contact manifold}

Let $(W, \beta)$ be a Liouville manifold. (See Section 2.1 for a definition and discussions.) Then the simplest contact manifold with convex/sutured boundary is the product sutured contact manifold

$$
(M, \Gamma, \alpha)=(W \times[-1,1], \partial W \times\{0\}, \operatorname{Ker}(d t+\beta)),
$$

where $t$ denotes the $[-1,1]$-coordinate on $W \times[-1,1]$.

Lemma 1.7 Suppose $(M, \Gamma, \xi)$ is a product sutured manifold. If $\alpha=d t+\beta$ is a $[-1,1]-$ invariant contact form for $\xi$ as above, then

(1) $H C(M, \Gamma, \xi)=\mathbb{Q}$;

(2) $\operatorname{ECH}(M, \Gamma, \alpha, J)=\mathbb{Z}$, if $\operatorname{dim} M=3$.

Proof The Reeb vector field of $\alpha$ is $R_{\alpha}=\partial_{t}$, which has no closed orbits. The algebra $H C(M, \Gamma, \alpha)=\mathbb{Q}$ is generated by the unit 1 , and the $\mathbb{Z}$-module $\operatorname{ECH}(M, \Gamma, \alpha, J)=\mathbb{Z}$ is generated by the empty set.

\subsection{Gluing theorems}

1.4.1 Connected sums The simplest gluing result describes the behavior of contact homology and ECH under connected sum. Given a $(2 n+1)$-dimensional closed contact manifold $(M, \xi)$, let us write $\widehat{H C}(M, \xi)=H C\left(M-B^{2 n+1}, \Gamma=S^{2 n-1},\left.\xi\right|_{M-B^{2 n+1}}\right)$, where $\left(B^{2 n+1}, \Gamma=S^{2 n-1}, \xi\right)$ is the standard Darboux ball with convex boundary. Then:

Theorem 1.8 Let $\left(M_{1}, \xi_{1}\right)$ and $\left(M_{2}, \xi_{2}\right)$ be $(2 n+1)$-dimensional closed contact manifolds. If $\left(M_{1} \# M_{2}, \xi_{1} \# \xi_{2}\right)$ is the contact manifold obtained by removing standard Darboux balls from each $\left(M_{i}, \xi_{i}\right)$ and gluing, then

(1) $\widehat{H C}\left(M_{1} \# M_{2}, \xi_{1} \# \xi_{2}\right)=\widehat{H C}\left(M_{1}, \xi_{1}\right) \otimes \widehat{H C}\left(M_{2}, \xi_{2}\right)$;

(2) if $\operatorname{dim} M_{1}=\operatorname{dim} M_{2}=3$ and we take $E C H$ with coefficients in a field, then

$$
\widehat{E C H}\left(M_{1} \# M_{2}, \xi_{1} \# \xi_{2}\right)=\widehat{E C H}\left(M_{1}, \xi_{1}\right) \otimes \widehat{E C H}\left(M_{2}, \xi_{2}\right) \text {. }
$$

The proof of Theorem 1.8 is given in Section 8.4. We remark that, in Theorem 1.8(2), we have a tensor product of homologies since the ground ring is a field. With $\mathbb{Z}$ coefficients one would need to modify the right hand side according to the Künneth formula for the homology of a tensor product of chain complexes. Note also that Theorem 1.8(b) is consistent with the conjectural equivalence of ECH and Heegaard Floer homology (and their respective $U$ maps), because the analogous property holds for $\widehat{H F}$. 
Before stating the next two theorems we need to make the following:

Disclaimers Theorems 1.9(2) and 1.10(2) for ECH presuppose part of Conjecture 1.2, namely that sutured ECH depends only on the contact structure and not on the contact form or almost complex structure. They also assume a slightly stronger conjecture, namely that a suitable "exact symplectic cobordism" between sutured contact 3manifolds induces a map on sutured ECH satisfying certain basic properties; see Section 10.4 for details. Analogous maps on ECH induced by exact symplectic cobordisms between closed contact 3-manifolds are constructed by Hutchings and Taubes [27], using Seiberg-Witten theory.

1.4.2 Sutured manifold gluing Let $\left(M^{\prime}, \Gamma^{\prime}, \xi^{\prime}\right)$ be a sutured contact manifold. Suppose there exist codimension zero Liouville submanifolds $P_{+} \subset R_{+}\left(\Gamma^{\prime}\right)$ and $P_{-} \subset R_{-}\left(\Gamma^{\prime}\right)$ which are symplectomorphic with respect to $d \alpha$, where $\alpha$ is a contact 1 -form for $\xi^{\prime}$, and the symplectomorphism takes $\left.\alpha\right|_{P_{+}}$to $\left.\alpha\right|_{P_{-}}$. Then we can glue $P_{+}$ and $P_{-}$to obtain a new sutured contact manifold $(M, \Gamma, \xi)$ with a properly embedded surface $P$ which is transverse to the Reeb flow. Details of this sutured manifold gluing - the inverse procedure of a sutured manifold decomposition, as defined by Gabai [14] in dimension 3 - will be given in Section 4.3. We then have the following:

Theorem 1.9 If $(M, \Gamma, \xi)$ is obtained from performing a sutured manifold gluing on $\left(M^{\prime}, \Gamma^{\prime}, \xi^{\prime}\right)$, then there are canonical injections

(1) $\Phi: H C\left(M^{\prime}, \Gamma^{\prime}, \xi^{\prime}\right) \hookrightarrow H C(M, \Gamma, \xi)$;

(2) $\Phi: E C H\left(M^{\prime}, \Gamma^{\prime}, \xi^{\prime}\right) \hookrightarrow E C H(M, \Gamma, \xi)$, when $\operatorname{dim} M=3$.

Moreover, the map (1) is a $\mathbb{Q}$-algebra homomorphism. In both cases the image coincides with the subgroup of (E)CH generated by Reeb orbits which do not intersect $P$.

Theorem 1.9 is analogous to a theorem of Juhász in the context of sutured Floer homology $[30 ; 31]$, namely that there is an injection

$$
\Phi: \operatorname{SFH}\left(M^{\prime}, \Gamma^{\prime}\right) \hookrightarrow \operatorname{SFH}(M, \Gamma)
$$

of sutured Floer homology modules. Its proof will be given in Section 8.4.

1.4.3 Convex gluing A more general type of gluing is that of gluing along a closed convex submanifold. Postponing the precise procedure for gluing along a convex submanifold $S$ until Section 4.4, we have the following results: 
Theorem 1.10 If $(M, \Gamma, \xi)$ is obtained from $\left(M^{\prime}, \Gamma^{\prime}, \xi^{\prime}\right)$ by gluing along a closed convex submanifold $S$, then there are canonical maps

(1) $\Phi: H C\left(M^{\prime}, \Gamma^{\prime}, \xi^{\prime}\right) \rightarrow H C(M, \Gamma, \xi)$;

(2) $\Phi: E C H\left(M^{\prime}, \Gamma^{\prime}, \xi^{\prime}\right) \rightarrow E C H(M, \Gamma, \xi)$, when $\operatorname{dim} M=3$.

Moreover, the map (1) is a $\mathbb{Q}$-algebra homomorphism.

The proof of Theorem 1.10 will be given in Section 11. Theorem 1.10 is analogous to a theorem of Honda, Kazez and Matić [22] for sutured Floer homology.

Unlike the case of a sutured manifold gluing, the convex gluing does not necessarily give an injection of the corresponding contact homology algebras. However, we still have the following:

Corollary 1.11 If $H C(M, \Gamma, \xi) \neq 0$, then $H C\left(M^{\prime}, \Gamma^{\prime}, \xi^{\prime}\right) \neq 0$.

Proof This is due to the fact that the gluing map

$$
\Phi: H C\left(M^{\prime}, \Gamma^{\prime}, \xi^{\prime}\right) \rightarrow H C(M, \Gamma, \xi)
$$

is a $\mathbb{Q}$-algebra homomorphism.

For example, if $M$ is closed and if $L$ is a Legendrian submanifold of $M$, then $M$ is obtained by gluing along the convex submanifold $\partial N(L)$. Thus we obtain:

Corollary 1.12 Let $L$ be a closed Legendrian submanifold of a closed contact manifold $(M, \xi)$. If $H C(M, \xi) \neq 0$, then $H C(M, \xi, L) \neq 0$.

In contrast to Corollary 1.12, the Legendrian contact homology - due to Chekanov [5] and Eliashberg in dimension three, and Ekholm, Etnyre and Sullivan [10] in higher dimensions - of a stabilized Legendrian submanifold always vanishes. On the other hand, let $\mathcal{A}$ be the contact homology differential graded algebra (DGA) for some choice of contact form $\alpha$ for $(M, \xi)$, almost complex structure $J$, and abstract perturbation. If $\mathcal{A}$ admits an augmentation, ie a chain map $\mathcal{A} \rightarrow \mathbb{Q}$ with the trivial differential for $\mathbb{Q}$, for example if $(M, \xi)$ has an exact symplectic filling, then $H C(M, \xi) \neq 0$.

In a sequel, we plan to prove gluing theorems for contact homology and embedded contact homology for the initial step in a sutured manifold hierarchy. 
Outline of the paper Sections 2-4 present the basic material on sutured contact manifolds. In Section 2 we introduce Liouville manifolds, convex submanifolds and sutured contact manifolds, and in Section 3 we introduce almost complex structures which are "tailored" to sutured contact manifolds. Section 4 collects the various operations that can be done with sutured contact manifolds - in particular we discuss switching between the sutured and convex boundary conditions and explain the sutured manifold gluing and convex gluing procedures. Then in Section 5 we prove the necessary compactness results for holomorphic curves in completions of sutured contact manifolds. In Section 6 we define sutured contact homology and sutured ECH and prove Theorem 1.1. Section 7 is devoted to the various invariants that can be defined via sutured contact homology: the "hat" versions of contact homology and ECH, Legendrian knot invariants and a transverse knot filtration. Finally, after some preliminary considerations on neckstretching in Sections 8 and 9, we prove Theorem 1.9 in Section 10 and Theorem 1.10 in Section 11.

\section{Sutured contact manifolds}

In this paper, when we refer to a $(2 n+1)$-dimensional contact manifold $(M, \xi)$, it is assumed that the ambient manifold $M$ is oriented, and the contact structure $\xi$ is cooriented by a global 1 -form $\alpha$ which is positive, ie satisfies $\alpha \wedge(d \alpha)^{n}>0$.

\subsection{Liouville manifolds}

Definition 2.1 A Liouville manifold (often also called a Liouville domain) is a pair $(W, \beta)$ consisting of a compact, oriented $2 n$-dimensional manifold $W$ with boundary and a 1 -form $\beta$ on $W$, where $\omega=d \beta$ is a positive symplectic form on $W$ and the Liouville vector field $Y$ given by $l_{Y} \omega=\beta$ is positively transverse to $\partial W$ (ie exits from $W$ along $\partial W$ ). It follows that the 1 -form $\beta_{0}=\left.\beta\right|_{\partial W}$ (this notation means $\beta$ pulled back to $\partial W)$ is a positive contact form on $\partial W$, whose kernel we denote by $\zeta$.

There is a neighborhood $N(\partial W)$ of $\partial W$ which can be written as $(-\varepsilon, 0] \times \partial W$, with coordinates $(\tau, x)$, where $Y=\partial_{\tau}, \beta=e^{\tau} \beta_{0}$, and $\partial W=\{0\} \times \partial W$. In other words, $(N(\partial W), d \beta)$ is locally symplectomorphic to the symplectization of $\beta_{0}$, with $Y=\partial_{\tau}$.

We briefly give a proof of this fact: Since $Y$ is transverse to $\partial W$, we take $\partial W=$ $\{0\} \times \partial W$ and $Y=\partial_{\tau}$. Then we can write $\beta=\beta_{\tau}+f d \tau$, where $\beta_{\tau}=\left.\beta\right|_{\{\tau\} \times \partial W}$ does not contain any $d \tau$-term. Then $d \beta=d_{x} \beta_{\tau}+d \tau \wedge d \beta_{\tau} / d \tau+d_{x} f \wedge d \tau$, where $d_{x}$ means $d$ in the $\partial W$-direction. The Liouville condition $l_{Y} d \beta=\beta$ implies that $d \beta_{\tau} / d \tau-d_{x} f=\beta_{\tau}+f d \tau$. Hence $f=0$ and $d \beta_{\tau} / d \tau=\beta_{\tau}$, implying $\beta_{\tau}=e^{\tau} \beta_{0}$. 
We write $(\widehat{W}, \widehat{\beta})$ to denote the completion of $(W, \beta)$, obtained by attaching the positive symplectization $\left([0, \infty) \times \partial W, e^{\tau} \beta_{0}\right)$.

Two Liouville 1 -forms $\beta^{0}$ and $\beta^{1}$ on $W$ are homotopic if there is a 1 -parameter family of Liouville 1 -forms $\beta^{t}, t \in[0,1]$, such that the corresponding Liouville vector field $Y^{t}$ on $N(\partial W)=(-\varepsilon, 0] \times \partial W$ is $\partial_{\tau}$. We can then complete the homotopy $\beta^{t}$ to $\widehat{W}$ by setting $\widehat{\beta}^{t}=e^{\tau} \beta_{0}^{t}$ on $[0, \infty) \times \partial W$, where $\beta_{0}^{t}=\left.\beta^{t}\right|_{\partial W}$.

\subsection{Convex submanifolds}

Let $(M, \xi)$ be a $(2 n+1)$-dimensional contact manifold. Following Giroux [15], we say that a closed, oriented $2 n$-dimensional submanifold $\Sigma$ of $M$ is $\xi$-convex if there is a contact vector field $X$ transverse to $\Sigma$. (Recall that a contact vector field is generated by a contact Hamiltonian function. Hence any contact vector field $X$ which is defined in a neighborhood of $\Sigma$ can be extended to a contact vector field on all of $M$, and thus convexity is a local condition.) Given $X$ as above, one defines the dividing set $\Gamma$ to be $\{x \in \Sigma \mid X(x) \in \xi(x)\}$.

To understand the dividing set more explicitly, let $N(\Sigma)=[-\varepsilon, \varepsilon] \times \Sigma$ be a neighborhood $^{2}$ of $\Sigma=\{0\} \times \Sigma$, such that $X=\partial_{t}$, where $t$ denotes the $[-\varepsilon, \varepsilon]$-coordinate. By changing the sign of $X$ if necessary, we may assume that $\partial_{t}$ gives the normal orientation of $\Sigma$. We can now find a 1 -form $\alpha$ for $\xi$ which in $N(\Sigma)$ is given by $\alpha=f d t+\beta$, where $f$ and $\beta$ do not depend on $t$ and $\beta$ has no $d t$-term. The dividing set is then $\Gamma=\{f=0\}$. Since $\alpha$ is a contact form,

$$
\alpha \wedge(d \alpha)^{n}=f d t(d \beta)^{n}+n d f d t \beta(d \beta)^{n-1}>0 .
$$

It follows that (i) $d f \neq 0$ along $\Gamma$, and hence $\Gamma$ is a codimension 1 submanifold of $\Sigma$, and (ii) $\beta$ is a contact form on $\Gamma$. In particular, (iii) $\xi=\operatorname{ker} \alpha$ is transverse to $\Gamma$. The dividing set $\Gamma$ is not necessarily connected.

Lemma 2.2 A closed, oriented, codimension one submanifold $\Sigma \subset(M, \xi)$ is $\xi$-convex if and only if there is an oriented, codimension one submanifold $\Gamma$ of $\Sigma$ and a (cooriented) contact form $\alpha$ for $\xi$ such that

(A) $\Gamma$ decomposes $\Sigma$ into alternating positive and negative open regions $R_{ \pm}(\Gamma)$ so that $\left(R_{+}(\Gamma),\left.d \alpha\right|_{R_{+}(\Gamma)}\right)$ and $\left(R_{-}(\Gamma),\left.d \alpha\right|_{R_{-}(\Gamma)}\right)$, endowed with the orientation of $\Sigma$ on $R_{+}(\Gamma)$ and its opposite on $R_{-}(\Gamma)$, are positive symplectic manifolds;

(B) the form $\left.\alpha\right|_{\Gamma}$ is a positive contact form on $\Gamma$ for the boundary orientation of $R_{+}(\Gamma)$.

\footnotetext{
${ }^{2}$ In this paper, a "neighborhood" is not necessarily an open neighborhood.
} 
A contact form $\alpha$ satisfying (A) and (B) above is said to be adapted to $(\Sigma, \Gamma)$. When $M$ has dimension three, $\Gamma$ is an oriented multicurve on the surface $\Sigma$ which is positively transverse to $\xi$.

Remark 2.3 Let $R_{\alpha}$ be the Reeb vector field associated with $\alpha$. The condition that $d \alpha$ be symplectic on $R_{ \pm}(\Gamma)$ is equivalent to the condition that $R_{\alpha}$ be positively transverse to $\Sigma$ along $R_{+}(\Gamma)$ and negatively transverse to $\Sigma$ along $R_{-}(\Gamma)$.

Remark 2.4 If $(\Sigma, \Gamma)$ is a convex hypersurface of $(M, \xi)$, then the proof of Lemma 2.2 shows that the closures $\overline{R_{ \pm}(\Gamma)}$ are Liouville manifolds with a Liouville form obtained from the restriction of an adapted contact form by a slight modification near $\Gamma$. Also, one can choose an adapted contact form $\alpha$ so that $\left(\Sigma,\left.d \alpha\right|_{\Sigma}\right)$ is a folded symplectic manifold, as defined in [34].

The proof of Lemma 2.2 uses the following notion: Given a codimension one submanifold $\Sigma$ of $(M, \xi)$, the characteristic line field $L$ is the singular line field in $\zeta=\xi \cap T \Sigma$ such that $\iota_{L}\left(\left.d \alpha\right|_{\zeta}\right)=0$ for any contact form $\alpha$ for $\xi$. The line field $L$ is singular where $\xi=T \Sigma$.

Proof of Lemma $2.2 \Leftrightarrow)$ Suppose $\Sigma$ is a convex submanifold. Let $\alpha=f d t+\beta$ be the contact form on $N(\Sigma)=[-\varepsilon, \varepsilon] \times \Sigma$ as above. By Equation (3), $\Gamma$ can be oriented so that $\left.\alpha\right|_{\Gamma}$ is a positive contact form on $\Gamma$. With this orientation of $\Gamma$, the normal orientation of $\Gamma$ in $\Sigma$ is given by the direction in which $f$ is decreasing. We then define $R_{+}(\Gamma)$ (resp. $\left.R_{-}(\Gamma)\right)$ to be the region $\{f>0\}$ (resp. $\{f<0\}$ ). This proves (B).

In order to prove (A) we further normalize the contact form. Take a sufficiently small neighborhood $N(\Gamma)=[-1,1] \times[-\varepsilon, \varepsilon] \times \Gamma$ of $\Gamma$ with coordinates $(\tau, t, x)$ so that $\beta$ is a contact form on all $\{(\tau, t)\} \times \Gamma$. Here we take $\partial_{t}$ for $N(\Gamma)$ to agree with $\partial_{t}$ for $N(\Sigma)$. By possibly multiplying $\alpha$ by a positive function, we may assume that $f=1$ for $\tau \geq \frac{1}{2}, f=-1$ for $\tau \leq-\frac{1}{2}, f$ is locally constant outside of $N(\Gamma)$, and $f=f(\tau)$ inside $N(\Gamma)$. Wherever $f$ is locally constant, $(d \beta)^{n}$ is $>0$ or $<0$ as appropriate, by Equation (3).

Next, let $L$ be the line field on $N(\Gamma)$ which agrees with the characteristic line field on each level set $A_{t_{0}}=\left\{t=t_{0}\right\}$ of $N(\Gamma)$. Take a $t$-invariant vector field $Y$ that directs $L$ so that the component of $Y$ in the $\tau$-direction is exactly $\partial_{\tau}$. This is possible since $\xi \pitchfork \Gamma$ and $d \alpha$ is nondegenerate on $\xi \cap T \Gamma$; hence $L$ must have a component transverse to $\Gamma$. Flowing along $Y$ starting on $\Gamma$ gives us a new coordinate function 
$x: N(\Gamma) \rightarrow \Gamma$ so that $\alpha=f d t+\beta$, where $\beta$ only has $d x$-terms and no $d \tau$-term, $f=f(\tau)$ as before, and $\alpha$ is $t$-invariant. Now

$$
d \alpha=f^{\prime}(\tau) d \tau d t+d \tau \frac{d \beta}{d \tau}+d_{x} \beta
$$

where $d_{x}$ is the derivative in the $x$-direction. Since $l_{\partial_{\tau}}\left(\left.d \alpha\right|_{\zeta}\right)=0$, it follows that $(d \beta / d \tau)(v)=0$ for all $\left.v \in \operatorname{ker} \beta\right|_{T \Sigma}$, or, equivalently, $d \beta / d \tau$ is a function times $\beta$. Hence, on $N(\Gamma)$, we can write

$$
\alpha=f(\tau) d t+g(\tau, x) \beta_{0},
$$

where $\beta_{0}=\left.\beta\right|_{\{(0,0)\} \times \Gamma}$ and $g>0$. Also, the contact condition implies that $\partial g / \partial \tau<0$ when $f=1$ and $\partial g / \partial \tau>0$ when $f=-1$.

Finally, let $h$ be a positive $t$-invariant function on $N(\Gamma)$ so that

(i) $h=g$ for $\tau \geq \frac{1}{2}$ and $\tau \leq-\frac{1}{2}$;

(ii) $\partial h / \partial \tau<0$ for $\tau>0$;

(iii) $\partial h / \partial \tau>0$ for $\tau<0$.

We claim now that condition (A) is fulfilled by a contact form that agrees with $(h / g) \alpha$ on $N(\Sigma)$. We need to check that $d\left(\left.(h / g) \alpha\right|_{\Sigma}\right)$ is a positive symplectic form on $R_{+}(\Gamma)$ and a negative symplectic form on $R_{-}(\Gamma)$. On the complement of $N(\Gamma)$, this follows from Equation (3) since $f$ is constant there. On $N(\Gamma)$, we have $\left.(h / g) \alpha\right|_{\Sigma}=h \beta_{0}$, and $d\left(h \beta_{0}\right)$ is symplectic on each of $R_{ \pm}(\Gamma)$ by (ii) and (iii).

$(\Leftarrow)$ Suppose now that there is a contact 1 -form $\alpha$ which is adapted to $(\Sigma, \Gamma)$. Let $\beta=\left.\alpha\right|_{\Sigma}$. We first normalize $\beta$ on $N(\Gamma) \cap \Sigma=\{t=0,-1 \leq \tau \leq 1\}$ : Let $X$ be the characteristic vector field on $N(\Gamma) \cap \Sigma$ so that its $\tau$-component is $\partial_{\tau}$. Flowing along $X$ (starting at $\tau=0$ ) gives us new coordinates $(\tau, x)$ so that $\beta(\tau, x)=g(\tau, x) \beta_{0}$, where $\beta_{0}=\left.\beta\right|_{\tau=0}$ and $g$ is a positive function. Moreover, since $d \beta>0$ is a positive symplectic form for $\tau>0$, it follows that $\partial g / \partial \tau<0$ on $\tau>0$; similarly, $\partial g / \partial \tau>0$ on $\tau<0$.

Next we construct a 1 -form $\tilde{\alpha}$ on $N(\Sigma)$ of the form

$$
\tilde{\alpha}(\tau, t, x)=\tilde{f} d t+\tilde{\beta},
$$

where $\tilde{f}$ and $\tilde{\beta}$ do not depend on $t$. The function $\tilde{f}: \Sigma \rightarrow \mathbb{R}$ is constant outside of $N(\Gamma)$ and can be written as $\tilde{f}(\tau)$ on $N(\Gamma)$ so that $\tilde{f}(\tau)=1$ for $\tau \geq \frac{1}{2}, \tilde{f}(\tau)=-1$ for $\tau \leq-\frac{1}{2}, \tilde{f}(0)=0$, and $\tilde{f}^{\prime}(\tau)>0$ for $-\frac{1}{2}<\tau<\frac{1}{2}$. The 1 -form $\widetilde{\beta}$ equals $\beta$ outside of $N(\Gamma)$ and equals $\tilde{g} \beta_{0}$ on $N(\Gamma)$, where $\tilde{g}(\tau, x)=g(\tau, x)$ near $\tau=-1,1$, $\tilde{g}(\tau, x)$ only depends on $\tau$ for $-\frac{1}{2} \leq \tau \leq \frac{1}{2}, \tilde{g}>0$ for all $\tau, \partial \widetilde{g} / \partial \tau<0$ on $\tau>0$, and $\partial \widetilde{g} / \partial \tau>0$ on $\tau<0$. 
The 1 -form $\tilde{\alpha}$ is clearly contact outside of $N(\Gamma)$. Inside $N(\Gamma)$ we compute that

$$
\tilde{\alpha} \wedge(d \widetilde{\alpha})^{n}=n\left(\frac{\partial \tilde{f}}{\partial \tau} \widetilde{g}-\tilde{f} \frac{\partial \widetilde{g}}{\partial \tau}\right) \widetilde{g}^{n-1} d \tau d t \beta_{0}\left(d \beta_{0}\right)^{n-1}>0 .
$$

Since $\alpha, \widetilde{\alpha}$ pull back to 1 -forms $\beta, \widetilde{\beta}$ which differ by a conformal factor on $\Sigma$, there is a local diffeomorphism which fixes $\Sigma$ and sends $\operatorname{ker} \alpha$ to $\operatorname{ker} \tilde{\alpha}$. Since $\Sigma$ is clearly convex with respect to $\tilde{\alpha}$, the same holds for $\alpha$.

The following is a corollary of the above proof:

Corollary 2.5 Given a convex submanifold $\Sigma$, one can choose a neighborhood $N(\Sigma)=[-\varepsilon, \varepsilon] \times \Sigma$ on which the contact structure is given by the kernel of a contact form $\alpha_{0}=f d t+\beta$ such that $f$ and $\beta$ do not depend on $t ; f= \pm 1$ on $R_{ \pm}(\Gamma)-N(\Gamma)$; $f=f(\tau)$ on $N(\Gamma)=[-1,1] \times[-\varepsilon, \varepsilon] \times \Gamma$; the zero set of $f$ is $\tau=0 ; \beta=g(\tau) \beta_{0}$ on $N(\Gamma) ; g(\tau)>0 ; \beta_{0}$ is a contact form on $\Gamma$; and $(\partial f / \partial \tau) g-f(\partial g / \partial \tau)>0$.

Recall that $t$ the first coordinate of $N(\Sigma)=[-\varepsilon, \varepsilon] \times \Sigma$ and $\tau$ is the first coordinate of $N(\Gamma)=[-1,1] \times[-\varepsilon, \varepsilon] \times \Gamma$.

Example 2.6 Let $(K, \theta)$ be a supporting open book for a closed $(M, \xi)$ and let $\alpha$ be a contact form for $\xi$ adapted to $(K, \theta)$ (as in Giroux [16]). Let $\Sigma$ be the submanifold of $M$ which is the union of (closures of) two pages of the open book that match up smoothly. Then $\Sigma$ is $\xi$-convex with dividing set $K$ and adapted form $\alpha$.

\subsection{Sutured contact manifolds}

Definition 2.7 A compact oriented manifold $M$ of dimension $m$ with boundary and corners is a sutured manifold if it comes with an oriented, not necessarily connected submanifold $\Gamma \subset \partial M$ of dimension $m-2$ (called the suture), together with a neighborhood $U(\Gamma)=[-1,0] \times[-1,1] \times \Gamma$ of $\Gamma=\{(0,0)\} \times \Gamma$ in $M$, with coordinates $(\tau, t) \in[-1,0] \times[-1,1]$, such that the following holds:

- $U \cap \partial M=(\{0\} \times[-1,1] \times \Gamma) \cup([-1,0] \times\{-1\} \times \Gamma) \cup([-1,0] \times\{1\} \times \Gamma)$.

- $\partial M-(\{0\} \times(-1,1) \times \Gamma)$ is the disjoint union of two submanifolds which we call $R_{-}(\Gamma)$ and $R_{+}(\Gamma),{ }^{3}$ where the orientation of $\partial M$ agrees with that of $R_{+}(\Gamma)$ and is opposite that of $R_{-}(\Gamma)$, and the orientation of $\Gamma$ agrees with the boundary orientation of $R_{ \pm}(\Gamma)$.

- The corners of $M$ are precisely $\{0\} \times\{ \pm 1\} \times \Gamma$.

${ }^{3}$ At the risk of some confusion, we will use this definition of $R_{ \pm}(\Gamma)$ when we view $(M, \Gamma)$ as a sutured manifold, and the definition of $R_{ \pm}(\Gamma)$ given in Section 2.2 when we think of $\partial M$ as being smooth. 
The notion of a sutured manifold was introduced by Gabai in [14] for 3-manifolds. The definition above is slightly different from the usual one; in particular the neighborhoods $U(\Gamma)$ do not appear in Gabai's definition.

By analogy with the theory of branched surfaces, often the submanifold $\partial_{h} M=$ $R_{+}(\Gamma) \cup R_{-}(\Gamma)$ is called the horizontal boundary and $\partial_{v} M=\{0\} \times[-1,1] \times \Gamma$ the vertical boundary of $M{ }^{4}$

Definition 2.8 Let $(M, \Gamma, U(\Gamma))$ be a $(2 n+1)$-dimensional sutured manifold. If $\xi$ is a contact structure on $M$, we say that $(M, \Gamma, U(\Gamma), \xi)$ is a sutured contact manifold if $\xi$ is the kernel of a positive contact 1 -form $\alpha$ such that

- $\left(R_{+}(\Gamma), \beta_{+}=\left.\alpha\right|_{R_{+}(\Gamma)}\right)$ and $\left(R_{-}(\Gamma), \beta_{-}=\left.\alpha\right|_{R_{-}(\Gamma)}\right)$ are Liouville manifolds;

- $\alpha=C d t+\beta$ inside $U(\Gamma)$, where $C$ is a positive constant and $\beta$ is independent of $t$ and does not have a $d t$-term;

- $\left.\partial_{\tau}\right|_{R_{ \pm}(\Gamma)}=Y_{ \pm}$inside $U(\Gamma)$, where $Y_{ \pm}$is the Liouville vector field for $\beta_{ \pm}$.

Such a contact form $\alpha$ is said to be adapted to $(M, \Gamma, U(\Gamma))$. (This is analogous to, but different from, the notion of a contact form adapted to a convex submanifold as discussed in Section 2.2.) We sometimes denote the sutured contact manifold by $(M, \Gamma, U(\Gamma), \alpha)$.

We note two immediate consequences of the above definition. First, the Reeb vector field $R_{\alpha}$ of $\alpha$ is equal to $(1 / C) \partial_{t}$ on $U(\Gamma)$ and is positively transverse to all of $R_{ \pm}(\Gamma)$, ie enters $M$ along $R_{-}(\Gamma)$ and exits $M$ along $R_{+}(\Gamma)$. This positive transversality holds because $\left.d \alpha\right|_{R_{ \pm}(\Gamma)}$ is symplectic. Second, on $U(\Gamma)=[-1,0] \times[-1,1] \times \Gamma$ with coordinates $(\tau, t, x)$, we have $\alpha=C d t+e^{\tau} \beta_{0}(x)$, where $\beta_{0}$ is a contact form on $\Gamma$.

Example 2.9 Let $(W, \beta)$ be a Liouville manifold and let $N(\partial W)=(-\varepsilon, 0] \times \partial W$ be the neighborhood of $\partial W$ with coordinates $(\tau, x)$, so that the Liouville vector field $Y$ equals $\partial_{\tau}$. Then the manifold

$$
(W \times[-1,1], \partial W \times\{0\}, N(\partial W) \times[-1,1], d t+\beta)
$$

is a sutured contact manifold, called a product sutured contact manifold.

\footnotetext{
${ }^{4}$ Strictly speaking, the orientation of $U(\Gamma)$ is that of the product $[-1,1] \times[-1,0] \times \Gamma$. However we write the first two factors in the opposite order because we want to visualize $[-1,0]$ as the horizontal direction and $[-1,1]$ as the vertical direction.
} 
Example 2.10 Let $\left(M^{\prime}, \Gamma^{\prime}, U\left(\Gamma^{\prime}\right), \xi^{\prime}\right)$ be a $(2 n+1)$-dimensional sutured contact manifold with adapted contact form $\alpha^{\prime}$. Let $\Gamma_{0}^{\prime} \subset \Gamma^{\prime}$ be a union of connected components of $\Gamma^{\prime}$. Also let $(W, \beta)$ be a $2 n$-dimensional Liouville cobordism from $\partial_{+} W$ to $\partial_{-} W$. By this we mean that $\partial W=\partial_{+} W-\partial_{-} W$ and $d \beta$ is a symplectic form on $W$, such that the Liouville vector field $Y$ satisfying $l_{Y} d \beta=\beta$ points into $W$ along $\partial_{-} W$ and out of $W$ along $\partial_{+} W$. Suppose there is a diffeomorphism

$$
\phi:\left(\partial_{-} W,\left.\beta\right|_{\partial_{-} W}\right) \stackrel{\sim}{\rightarrow}\left(\Gamma_{0}^{\prime},\left.\beta_{0}\right|_{\Gamma_{0}^{\prime}}\right) .
$$

We can then define a new sutured contact manifold $(M, \Gamma, U(\Gamma), \xi)$, called an intervalfibered extension of $\left(M^{\prime}, \Gamma^{\prime}, U\left(\Gamma^{\prime}\right), \xi^{\prime}\right)$, by

$$
M=M^{\prime} \sqcup(W \times[-1,1]) / \sim,
$$

where $(0, t, \phi(y)) \sim(y, t)$ for all $y \in \partial_{-} W$. Here $\xi=\operatorname{Ker}(\alpha)$ where $\alpha$ is obtained by gluing $\alpha^{\prime}$ and $C d t+\beta$. Also $\Gamma=\left(\Gamma^{\prime}-\Gamma_{0}^{\prime}\right) \sqcup\left(\partial_{+} W \times\{0\}\right)$, and $R_{ \pm}(\Gamma)=$ $R_{ \pm}\left(\Gamma^{\prime}\right) \cup(W \times\{ \pm 1\})$.

\subsection{Completion of a sutured contact manifold}

Let $(M, \Gamma, U(\Gamma), \xi)$ be a sutured contact manifold with an adapted contact form $\alpha$. We now explain how to extend $(M, \alpha)$ to a "complete" noncompact contact manifold $\left(M^{*}, \alpha^{*}\right)$.

The Reeb flow of $\alpha$ defines neighborhoods $[1-\varepsilon, 1] \times R_{+}(\Gamma)$ and $[-1,-1+\varepsilon] \times R_{-}(\Gamma)$ of $R_{+}(\Gamma)=\{1\} \times R_{+}(\Gamma)$ and $R_{-}(\Gamma)=\{-1\} \times R_{-}(\Gamma)$ respectively, in which $\alpha=$ $C d t+\beta_{ \pm}$, where $t \in[-1,-1+\varepsilon] \cup[1-\varepsilon, 1]$ extends the $t$-coordinate on $U(\Gamma)$. The first step is to extend $\alpha$ "vertically" by gluing $[1, \infty) \times R_{+}(\Gamma)$ and $(-\infty,-1] \times R_{-}(\Gamma)$ with the forms $C d t+\beta_{+}$and $C d t+\beta_{-}$respectively. The boundary of this new manifold is $\{0\} \times \mathbb{R} \times \Gamma$. In the neighborhood $[-1,0] \times \mathbb{R} \times \Gamma$ of the boundary with coordinates $(\tau, t, x)$, we have $\alpha=C d t+e^{\tau} \beta_{0}(x)$ where $\beta_{0}$ is a contact form on $\Gamma$.

To complete the construction of $\left(M^{*}, \alpha^{*}\right)$, we then extend "horizontally", similarly to the construction of an interval-fibered extension, by gluing $[0, \infty) \times \mathbb{R} \times \Gamma$ with the form $C d t+e^{\tau} \beta_{0}$.

For convenience, we extend the coordinates $(\tau, t)$ to functions on all of $M^{*}$ so that $t(M) \subset[-1,1]$ and $\tau\left(M \cup\left([1, \infty) \times R_{+}(\Gamma)\right) \cup\left((-\infty,-1] \times R_{-}(\Gamma)\right)\right) \subset[-1,0]$. We then refer to $t>1$ as the Top (T), to $t<-1$ as the Bottom (B), and to $\tau>0$ as the Side (S). Consistently with our notation for the completion of Liouville manifolds in general, we let $\left(\widehat{R_{ \pm}(\Gamma)}, \hat{\beta}_{ \pm}\right)$denote the completion of $\left(R_{ \pm}(\Gamma), \beta_{ \pm}\right)$obtained by extending to $(\mathrm{S})$. 


\section{Almost complex structures}

\subsection{Adapted and tailored almost complex structures}

Let $(Y, \xi)$ be a contact manifold. Then an almost complex structure $J$ on the symplectization $\mathbb{R} \times Y$, with $\mathbb{R}$-coordinate $s$, is adapted to the symplectization if the following hold:

(1) $J$ is $s$-invariant.

(2) $J$ takes $\xi$ to itself on each $\{s\} \times Y$.

(3) $J$ maps $\partial_{s}$ to the Reeb vector field $R_{\alpha}$ associated to a contact 1-form $\alpha$ for $\xi$.

(4) $\left.J\right|_{\xi}$ is $d \alpha$-positive, ie $d \alpha(v, J v)>0$ for all nonzero $v \in \xi$.

We remark that Condition (4) does not depend on the choice of $\alpha$. If we also want to specify the contact 1 -form $\alpha$, then we say that $J$ is $\alpha$-adapted.

Let $(W, \beta)$ be a Liouville manifold and let $\zeta$ be the contact structure given on $\partial W$ by $\operatorname{ker} \beta_{0}$, where $\beta_{0}=\left.\beta\right|_{\partial W}$. Recall the completion $(\widehat{W}, \hat{\beta})$ of $(W, \beta)$, where $\widehat{W}=W \cup([0, \infty) \times \partial W)$ and $\left.\widehat{\beta}\right|_{[0, \infty) \times \partial W}=e^{\tau} \beta_{0}$. Here $\tau$ is the $[0, \infty)$-coordinate. An almost complex structure $J_{0}$ on $\widehat{W}$ is $\widehat{\beta}$-adapted if it is

(1) $\beta_{0}$-adapted on $[0, \infty) \times \partial W$;

(2) $d \beta$-positive on $W$, ie $d \beta\left(v, J_{0} v\right)>0$ for all nonzero tangent vectors $v$.

Let $(M, \Gamma, U(\Gamma), \xi)$ be a sutured contact manifold, $\alpha$ an adapted contact form and $\left(M^{*}, \alpha^{*}\right)$ its completion. We consider the symplectization $\left(\mathbb{R} \times M^{*}, d\left(e^{s} \alpha^{*}\right)\right)$ of $\left(M^{*}, \alpha^{*}\right)$, where $s$ is the coordinate on $\mathbb{R}$. We say that an almost complex structure $J$ on $\mathbb{R} \times M^{*}$ is tailored to $\left(M^{*}, \alpha^{*}\right)$ if the following hold:

( $\left.\mathrm{A}_{0}\right) \quad J$ is $\alpha^{*}$-adapted.

(A $\left.\mathrm{A}_{1}\right) \quad J$ is $\partial_{t}$-invariant in a neighborhood of $\mathbb{R} \times\left(M^{*} \backslash \operatorname{int}(M)\right)$.

$\left(\mathrm{A}_{2}\right)$ The projection of $J$ to $T \widehat{R_{ \pm}(\Gamma)}$ is a $\hat{\beta}_{ \pm}$-adapted almost complex structure $J_{0}$ on the completion $\left(\widehat{R_{+}(\Gamma)}, \hat{\beta}_{+}\right) \sqcup\left(\widehat{R_{-}(\Gamma)}, \hat{\beta}_{-}\right)$of the Liouville manifold $\left(R_{+}(\Gamma), \beta_{+}\right) \sqcup\left(R_{-}(\Gamma), \beta_{-}\right)$.

Note that, by the above conditions, $J_{0}$ uniquely determines $J$ on $\mathbb{R} \times\left(M^{*} \backslash \operatorname{int}(M)\right)$. Moreover, the flow of $\partial_{t}$ identifies $J_{0} \mid \widehat{R_{+}(\Gamma)}-R_{+}(\Gamma)$ and $J_{0} \mid \widehat{R_{-}(\Gamma)}-R_{-}(\Gamma)$. 


\subsection{Integrable complex structures $J_{0}$ for Stein domains}

We now discuss the integrable complex structure on a Stein domain, which, as we will see in Section 5.3, is often more convenient for calculations. The slight drawback is that the integrable complex structure is usually not adapted to the symplectization.

Let $\left(W, J_{0}\right)$ be a Stein domain. Then there exists a Morse function $f: W \rightarrow \mathbb{R}$ which is strictly plurisubharmonic and for which $\partial W$ is a regular level set. If $\beta=-d^{\mathbb{C}} f=$ $-d f \circ J_{0}$, then we claim that $(W, \beta)$ is a Liouville manifold and that the symplectic form $\omega=d \beta$ is $J_{0}$-compatible. Indeed, $\omega$ is symplectic since $\omega\left(v, J_{0} v\right)>0$ (ie $\omega$ is tamed by $J_{0}$ ) for all nonzero tangent vectors $v$ of $W$ by the strict plurisubharmonicity of $f$. Moreover, $\omega\left(\cdot, J_{0} \cdot\right)$ is symmetric by the integrability of $J_{0}$ : Writing

$$
(*)=-\omega\left(X, J_{0} Y\right)+\omega\left(Y, J_{0} X\right),
$$

we compute, using the formula $d \eta(A, B)=A(\eta(B))-A(\eta(B))-\eta([A, B])$, where $\eta$ is a $1-$ form and $A, B$ are vector fields, that

$$
\begin{aligned}
(*)=d d^{\mathbb{C}} f\left(X, J_{0} Y\right)+ & d d^{\mathbb{C}} f\left(J_{0} X, Y\right) \\
=X\left(d^{\mathbb{C}} f\left(J_{0} Y\right)\right)- & J_{0} Y\left(d^{\mathbb{C}} f(X)\right)-d^{\mathbb{C}} f\left(\left[X, J_{0} Y\right]\right) \\
& +J_{0} X\left(d^{\mathbb{C}} f(Y)\right)-Y\left(d^{\mathbb{C}} f\left(J_{0} X\right)\right)-d^{\mathbb{C}} f\left(\left[J_{0} X, Y\right]\right) .
\end{aligned}
$$

Now, the integrability of $J_{0}$ is equivalent to the vanishing of the Nijenhuis tensor, ie

$$
J_{0}\left[X, J_{0} Y\right]+J_{0}\left[J_{0} X, Y\right]=\left[J_{0} X, J_{0} Y\right]-[X, Y] .
$$

So $(*)=-X(d f(Y))+Y(d f(X))+d f([X, Y])+J_{0} X\left(d f\left(J_{0} Y\right)\right)-J_{0} Y\left(d f\left(J_{0} X\right)\right)-$ $d f\left(\left[J_{0} X, J_{0} Y\right]\right)=-d^{2} f(X, Y)+d^{2} f\left(J_{0} X, J_{0} Y\right)=0$, and we have proved that $\omega\left(\cdot, J_{0} \cdot\right)$ is symmetric. Now let $\zeta$ be the contact structure on $\partial W$ given by $\left.\operatorname{ker} \beta\right|_{\partial W}$. If $v \in \zeta$, then $\beta\left(J_{0} v\right)=d f(v)=0$, and thus $J_{0}$ fixes $\zeta$. Let $g(X, Y)=\omega\left(X, J_{0} Y\right)$ be the compatible metric on $W$. Then the Liouville vector field $X$ satisfying $\imath_{X} \omega=\beta$ is given by $X=\nabla f=J_{0} X_{f}$, where the gradient $\nabla$ is with respect to $g$ and $X_{f}$ is the Hamiltonian vector field of $f$ with respect to $\omega$. Hence the Liouville vector field $X$ is positively transverse to $\partial W$ and $(W, \beta)$ satisfies the conditions of a Liouville manifold.

When $W$ is a compact surface with nonempty boundary, there is a complex structure $J_{0}$ which makes $\left(W, J_{0}\right)$ into a Stein domain. Thus $W$ has the structure of a Liouville manifold with a compatible almost complex structure $J_{0}$.

One subtlety that we address in Section 3.4 is that, in a neighborhood of $\partial W$, the integrable $J_{0}$ is often slightly different from an almost complex structure $J_{0}^{\prime}$ which is $\beta_{0}=\left.\beta\right|_{\partial W}$-adapted. If $(-\varepsilon, \varepsilon) \times \partial W$ is a piece of the symplectization of $\partial W$ with coordinates $(\tau, x)$ and $\partial W=\{\tau=0\}$ so that the Liouville vector field $X=\partial_{\tau}$, then 
the level sets of $f$ differ slightly from the level sets of $\tau$. Also, while $J_{0}^{\prime}$ can be made to agree with $J_{0}$ on $\operatorname{ker} \beta_{0}$, and $J_{0}$ maps $\partial_{\tau} \mapsto g_{0} R_{\beta_{0}}$, the function $g_{0}$ is usually not constant. The following is an example of the above-mentioned issues, which the authors learned from Jian He.

Example 3.1 Consider $\mathbb{C}^{n}$ with coordinates $z_{i}=x_{i}+\sqrt{-1} y_{i}$ and the standard integrable complex structure $J_{0}$. Let $M$ be an ellipsoid in $\mathbb{C}^{n}$ which is a level set of

$$
f=\frac{1}{2} \sum_{i}\left(x_{i}^{2}+\lambda_{i} y_{i}^{2}\right)
$$

for some positive real numbers $\lambda_{i}$. We compute that

$$
\begin{gathered}
d f=\sum_{i}\left(x_{i} d x_{i}+\lambda_{i} y_{i} d y_{i}\right), \\
\beta=-d f \circ J_{0}=\sum_{i}\left(-\lambda_{i} y_{i} d x_{i}+x_{i} d y_{i}\right), \\
\omega=d \beta=\sum_{i}\left(1+\lambda_{i}\right) d x_{i} d y_{i}, \\
X_{f}=\sum_{i} \frac{1}{1+\lambda_{i}}\left(-x_{i} \partial_{y_{i}}+\lambda_{i} y_{i} \partial_{x_{i}}\right),
\end{gathered}
$$

where $X_{f}$ is the Hamiltonian vector field of $f$ with respect to $\omega$, and

$$
X=\nabla f=J_{0} X_{f}=\sum_{i} \frac{1}{1+\lambda_{i}}\left(x_{i} \partial_{x_{i}}+\lambda_{i} y_{i} \partial_{y_{i}}\right) .
$$

Hence, we have

$$
d f(X)=\sum_{i} \frac{1}{1+\lambda_{i}}\left(x_{i}^{2}+\lambda_{i}^{2} y_{i}^{2}\right) .
$$

It follows that if not all the $\lambda_{i}$ are the same, then $d f(X)=d f\left(\partial_{\tau}\right)$ is not constant on the level sets of $f$, and so the level sets of $\tau$ are different from the level sets of $f$.

\subsection{Interpolation of almost complex structures on symplectizations}

Let $Y$ be an odd-dimensional manifold and let $\beta_{0}, \beta_{0}^{\prime}$ be homotopic contact 1 -forms on $Y$, ie suppose there is a 1 -parameter family of contact 1 -forms from $\beta_{0}$ to $\beta_{0}^{\prime}$. Consider $\mathbb{R} \times Y$ with coordinates $(\tau, x)$. We then have the following lemma, which is used to prove that the sutured contact homology algebras are independent of the choice of almost complex structure: 
Lemma 3.2 There is a constant $C>0$ and an almost complex structure $J$ on $\mathbb{R} \times Y$ which is $C \cdot \beta_{0}^{\prime}$-adapted for $\tau \geq 1$ and $\beta_{0}$-adapted for $\tau \leq 0$, and such that $\tau$ is plurisubharmonic with respect to $J$, ie $-d d^{\mathbb{C}} \tau(v, J v)=-d(d \tau \circ J)(v, J v) \geq 0$ for all tangent vectors $v \in T(\mathbb{R} \times Y)$.

Proof This is just a modification of the usual proof of the plurisubharmonicity of $\tau$ with respect to a $\beta_{0}$-adapted almost complex structure.

By Gray's theorem, the homotopy from $\beta_{0}$ to $\beta_{0}^{\prime}$ gives rise to a diffeomorphism isotopic to the identity, which takes $\beta_{0}^{\prime}$ to $f \beta_{0}$ for some positive function $f$ on $Y$. Hence, after composing with a diffeomorphism of $\mathbb{R} \times Y$ of type $(\tau, x) \mapsto\left(\tau, \phi_{\tau}(x)\right)$, where $\phi_{\tau}: Y \stackrel{\sim}{\rightarrow} Y$ is a diffeomorphism, we may assume that $\beta_{0}^{\prime}=f \beta_{0}$. We then define a 1 -form $\beta(\tau, x)=g(\tau, x) \beta_{0}(x)$ on $\mathbb{R} \times Y$ such that $g(\tau, x)$ is a smooth function which satisfies the following:

(i) $g(\tau, x)=1$ for $\tau \leq 0$.

(ii) $g(\tau, x)=C \cdot f(x)$ for $\tau \geq 1$, where $C$ is a constant greater than $\max (1 / f)$.

(iii) $\partial g(\tau, x) / \partial \tau \geq 0$.

Let $R_{\tau}$ be the Reeb vector field for $\beta(\tau)$. Then we choose $J$ so that $J(\tau, x)$ sends $\operatorname{ker} \beta(\tau)=\operatorname{ker} \beta_{0}$ to itself and $\partial_{\tau}$ to $R_{\tau}$, and satisfies $d_{Y} \beta(\tau)(X, J X)>0$ for all nonzero $X \in \operatorname{ker} \beta(\tau)$, where $d_{Y}$ denotes the exterior derivative on $Y$.

We claim that $d \tau \circ J=-\beta$. Indeed, $d \tau \circ J$ sends $\operatorname{ker} \beta(\tau) \mapsto 0, \partial_{\tau} \mapsto 0$, and $R_{\tau} \mapsto-1$, agreeing with the evaluation of $-\beta$ on these tangent vectors.

We now have

$$
-d d^{\mathbb{C}} \tau=d \beta=d\left(g \beta_{0}\right)=\frac{\partial g}{\partial \tau} d \tau \wedge \beta_{0}+d_{Y}\left(g \beta_{0}\right),
$$

If we write $v \in T(\mathbb{R} \times Y)$ as $X+a \partial_{\tau}+b R_{\tau}$, where $X \in \operatorname{ker} \beta(\tau)$, then $J v=$ $J X+a R_{\tau}-b \partial_{\tau}$. Evaluating the pair $(v, J v)$ on the right-hand side of Equation (6), we obtain

$$
g^{-1} \frac{\partial g}{\partial \tau}\left(a^{2}+b^{2}\right)+d_{Y}\left(g \beta_{0}\right)(X, J X) \geq 0 .
$$

This proves the plurisubharmonicity of $\tau$.

By rescaling in the $\tau$-direction we obtain the following:

Corollary 3.3 There is an almost complex structure $J$ on $\mathbb{R} \times Y$ which is $\beta_{0}^{\prime}$-adapted for sufficiently positive $\tau$ and $\beta_{0}$-adapted for sufficiently negative $\tau$, so that some increasing function $u$ of $\tau$ is $J$-plurisubharmonic. In particular, no holomorphic map from a Riemann surface with punctures into $(\mathbb{R} \times Y, J)$ attains a local maximum in the $\tau$-direction. 


\subsection{Interpolation between the adapted and integrable almost complex structures}

Let $\left(W, J_{0}\right)$ be a Stein domain with a strictly plurisubharmonic function $\tau$ and a corresponding Liouville 1 -form $\beta$. (Unlike our previous notation, $\tau$ now denotes the plurisubharmonic function and not the coordinate near the boundary given by the Liouville vector field.) Without loss of generality, we may assume that $\partial W=\{\tau=0\}$. Writing $Y=\partial W$, let $N(\partial W)=[-\varepsilon, 0] \times Y$ be a neighborhood of $\partial W=\{0\} \times Y$ with coordinates $(\tau, x)$. Extend this to $[-\varepsilon, \infty) \times Y$, also with coordinates $(\tau, x)$. Write $\beta_{\tau}=\left.\beta\right|_{\{\tau\} \times Y}$ and $\zeta_{\tau}=\operatorname{ker} \beta_{\tau}$.

Lemma 3.4 Suppose $\beta_{0}^{\prime}$ is a contact 1 -form homotopic to $\beta_{0}$. On $[-\varepsilon, \infty) \times Y$, there exist an almost complex structure $J$ and a $J$-plurisubharmonic function $u(\tau)$ such that

(i) $J$ is $\beta_{0}^{\prime}$-adapted for sufficiently positive $\tau$;

(ii) $J$ agrees with $J_{0}$ on $N(\partial W)$.

We thank Yasha Eliashberg for suggesting that something like the above lemma might be true.

Proof By applying Corollary 3.3 above, we may assume that $\beta_{0}^{\prime}=\beta_{0}$.

Let us first consider the Liouville 1 -form $\beta=-d \tau \circ J_{0}$ on $N(\partial W)$. By changing the identification of $N(\partial W)$ with $[-\varepsilon, 0] \times Y$, we can arrange for the vector field $\partial_{\tau}$ to be parallel to, but not necessarily a constant multiple of, the Liouville vector field $\nabla \tau$ which satisfies $\iota_{\nabla \tau} d \beta=\beta$. It then follows that $\beta$ has no $d \tau$-terms. Hence $\beta(\tau, x)=\beta_{\tau}(x)$. We also observe that, if $R_{\tau}$ is the Reeb vector field for $\beta_{\tau}$ on $\{\tau\} \times Y$, then it is parallel to the Hamiltonian vector field $X_{\tau}$ for $\tau$, which satisfies

$$
l_{X_{\tau}} d \beta=l_{X_{\tau}}\left(d_{Y} \beta_{\tau}+d \tau \wedge \dot{\beta}_{\tau}\right)=d \tau,
$$

where $\dot{\beta}_{\tau}=d \beta_{\tau} / d \tau$. Moreover, we claim that $J_{0}\left(\partial_{\tau}\right)=R_{\tau}$. Indeed, since $\nabla \tau$ is parallel to $\partial_{\tau}, X_{\tau}$ is parallel to $R_{\tau}$, and $J_{0}(\nabla \tau)=-X_{\tau}$, we have $J_{0}\left(\partial_{\tau}\right)$ is a function times $R_{\tau}$. The function can be determined from the equation $\beta_{\tau}\left(R_{\tau}\right)=$ $-d \tau \circ J_{0}\left(R_{\tau}\right)=1$.

Next define a smooth function $u:[-\varepsilon, 0] \rightarrow \mathbb{R}$ so that it satisfies

- $u(\tau)=\tau$ on $\left[-\varepsilon,-\frac{\varepsilon}{2}\right]$

- $d^{2} u / d \tau^{2} \geq 0$ on $[-\varepsilon, 0]$;

- $\left(d^{2} u / d \tau^{2}\right)(0) \gg(d u / d \tau)(0)$. 
The function $u(\tau)$ is $J_{0}$-plurisubharmonic on $N(\partial W)$. This follows from the general fact that the composition of a plurisubharmonic function with a smooth, increasing, convex function $u$ from a subset of $\mathbb{R}$ to $\mathbb{R}$ is plurisubharmonic. Here "convex" means $u^{\prime \prime} \geq 0$ at all points in the domain. To see this explicitly, if we set $\beta^{\prime}=-d u \circ J_{0}$, then

$$
\begin{aligned}
\beta^{\prime} & =-\frac{d u}{d \tau}\left(d \tau \circ J_{0}\right)=\frac{d u}{d \tau} \beta, \\
d \beta^{\prime} & =\frac{d^{2} u}{d \tau^{2}} d \tau \wedge \beta+\frac{d u}{d \tau} d \beta \\
& =\frac{d^{2} u}{d \tau^{2}} d \tau \wedge\left(-d \tau \circ J_{0}\right)+\frac{d u}{d \tau} d \beta .
\end{aligned}
$$

The conditions on $u(\tau)$ then imply that $d \beta^{\prime}\left(v, J_{0} v\right)>0$ for all nonzero $v$.

It is useful below to write $g(\tau)=d u / d \tau$, and to rewrite the above equation as

$$
d \beta^{\prime}=\frac{d g}{d \tau} d \tau \wedge\left(-d \tau \circ J_{0}\right)+g\left(d_{Y} \beta_{\tau}+d \tau \wedge \dot{\beta}_{\tau}\right)
$$

where $g(\tau)$ satisfies $d g / d \tau \gg g$ near $\tau=0$.

We now extend $\beta^{\prime}=g \beta$ and $J=J_{0}$ over $[0, \infty) \times Y$. First choose $g:[0, \infty) \rightarrow \mathbb{R}$ so that $d g / d \tau \gg g$ on $[0,1]$ and $d g / d \tau>0$ elsewhere. We then extend $\beta$ so that

- $\beta(\tau, x)=\beta_{\tau}$, ie $\beta$ has no $d \tau$-term;

- $\beta_{\tau}$ are contact forms on $Y$;

- $\beta_{\tau}=\beta_{0}$ for $\tau \geq 1$.

(The only reason we cannot set $\beta_{\tau}=\beta_{0}$ for all $\tau \geq 0$ is that we require $\beta$ to be smooth.) Let $\zeta_{\tau}=\operatorname{ker} \beta_{\tau}$ and $R_{\tau}=R_{\beta_{\tau}}$. Since $J_{0}$ maps $\zeta_{\tau}$ to itself and $\partial_{\tau} \mapsto R_{\tau}$ on $N(\partial W)$, we can extend $J_{0}$ to $J$ so that $\zeta_{\tau}$ is mapped to itself and $\partial_{\tau} \mapsto R_{\tau}$.

Now let $u=u(\tau)$ be the extension of $\left.u\right|_{N(\partial W)}$ to $[-\varepsilon, \infty) \times Y$ so that $d u / d \tau=g(\tau)$. To show that $u$ is $J$-plurisubharmonic, first observe that

$$
-d u \circ J=\frac{d u}{d \tau}(-d \tau \circ J)=g \beta=\beta^{\prime} .
$$

Thus we need to verify the nonnegativity condition $d \beta^{\prime}(v, J v) \geq 0$. Write $v=$ $X+a \partial_{\tau}+b R_{\tau}$, where $X \in \zeta_{\tau}$, so that $J v=J X+a R_{\tau}-b \partial_{\tau}$. Then Equation (8) gives

$d \beta^{\prime}(v, J v)=\frac{d g}{d \tau}\left(a^{2}+b^{2}\right)+g\left(d_{Y} \beta_{\tau}(X, J X)\right)$

$$
+g\left(a \dot{\beta}_{\tau}\left(J X+a R_{\tau}\right)+b \dot{\beta}_{\tau}\left(X+b R_{\tau}\right)\right) .
$$


The nonnegativity is immediate for $\tau \geq 1$ since $\dot{\beta}_{\tau}=0$. The nonnegativity for $\tau \in[0,1]$ follows from $d g / d \tau \gg g$ and is based on the inequality

$$
k \sum_{i} x_{i}^{2}+K \sum_{j} y_{j}^{2} \geq \sum_{i j} a_{i j} x_{i} y_{j},
$$

where $k>0$ and $a_{i j}$ are given, and $K \gg 0$ is chosen in response to $k, a_{i j}$. In the application of this inequality, the numbers $x_{i}$ are the components of $X$ in some local coordinate system, while the numbers $y_{j}$ are $a$ and $b$.

\section{Operations on sutured contact manifolds}

In this section we collect some operations that are done to sutured contact manifolds.

Roadmap Section 4.3 and Section 4.4 give some technical constructions which will only be used in Sections 10 and 11. The first-time reader may want to skip those sections and proceed to Section 5.

\subsection{Switching between convex and sutured boundary conditions}

In this section we describe how to pass between the convex and sutured boundary conditions.

When $(M, \Gamma, U(\Gamma), \xi)$ is a sutured contact manifold, it is easy to smooth the corners of $M$ inside $U(\Gamma)=[-1,0] \times[-1,1] \times \Gamma$, so that the resulting manifold $M^{\prime}$ has boundary $\partial M^{\prime}$ which is transverse to the Reeb vector field $R=(1 / C) \partial_{t}$ except at $\Gamma=\{(0,0)\} \times \Gamma$. More precisely, the portion of $\partial M^{\prime}$ for which $t>0$ (resp. $\left.t<0\right)$ is positively (resp. negatively) transverse to $R$. Hence the slight retract $\left(M^{\prime}, \Gamma,\left.\xi\right|_{M^{\prime}}\right)$ of $M$ has $\xi$-convex boundary by Lemma 2.2.

On the other hand, the following lemma explains how to pass from convex to sutured boundary.

Lemma 4.1 Let $(M, \xi)$ be a $(2 n+1)$-dimensional contact manifold with $\xi$-convex boundary $(\partial M, \Gamma)$, and let $N(\Gamma) \subset M$ be a tubular neighborhood of $\Gamma$. Then there exists a codimension 0 sutured contact submanifold $\left(M^{\prime}, \Gamma^{\prime}, U\left(\Gamma^{\prime}\right),\left.\xi\right|_{M^{\prime}}\right)$ of $M$, together with a contact form $\alpha$ on $M$, such that $\left.\alpha\right|_{M^{\prime}}$ is adapted to $\left(M^{\prime}, \Gamma^{\prime}, U\left(\Gamma^{\prime}\right),\left.\xi\right|_{M^{\prime}}\right)$, $M-M^{\prime} \subset N(\Gamma), U\left(\Gamma^{\prime}\right) \subset N(\Gamma)$, and $\left(\Gamma^{\prime}, \xi \cap T \Gamma^{\prime}\right)$ is isotopic to $(\Gamma, \xi \cap T \Gamma)$ through $(2 n-1)$-dimensional contact submanifolds of $(M, \xi)$. 
Proof Since $\Sigma=\partial M$ is $\xi$-convex, there is a neighborhood $N(\Sigma)=[-\varepsilon, 0] \times \Sigma$ of $\Sigma=\{0\} \times \Sigma$ with first coordinate $t$ and a contact form $\alpha_{0}=f d t+\beta$ as given by Corollary 2.5. In particular, on $N(\Gamma)=[-1,1] \times[-\varepsilon, 0] \times \Gamma$, the form $\alpha_{0}$ can be written as

$$
\alpha_{0}=f(\tau) d t+g(\tau) \beta_{0}=g(\tau)\left(\beta_{0}+\tilde{f}(\tau) d t\right) ;
$$

we may assume that $\tilde{f}(\tau)=\tau$ for $-\frac{1}{4} \leq \tau \leq \frac{1}{4}, \partial g / \partial \tau>0$ for $\tau<0, \partial g / \partial \tau<0$ for $\tau>0$, and $g(\tau)=g(-\tau)$. Then

(内) $R_{\alpha_{0}}$ is positively transverse to $\partial M$ along $R_{+}(\Gamma)$ and negatively transverse to $\partial M$ along $R_{-}(\Gamma)$.

Consider cylindrical coordinates $(r, \theta, x)$ on $N(\Gamma)$ so that

$$
(\tau, t)=(r \cos (\theta), r \sin (\theta))
$$

and the portion contained in $M$ is $\pi \leq \theta \leq 2 \pi$. Let

$$
U=\{\pi \leq \theta \leq 2 \pi, 0 \leq r \leq \delta\} \subset N(\Gamma) .
$$

Along $t=0,-\frac{1}{4} \leq \tau \leq \frac{1}{4}$, the contact forms

$$
\begin{aligned}
& \alpha_{0}=g(\tau)\left(\beta_{0}+\tilde{f}(\tau) d t\right), \\
& \alpha_{1}=g(r)\left(\beta_{0}+r^{2} d \theta\right)
\end{aligned}
$$

agree and the interpolation $\alpha_{s}=(1-s) \alpha_{0}+s \alpha_{1}$ is contact. Hence, by the usual MoserWeinstein technique, there is a 1-parameter family of local diffeomorphisms $\phi_{s}$, $s \in[0,1]$, near $\Gamma$ so that $\phi_{0}=\mathrm{id}, \phi_{s}=\mathrm{id}$ along $\Sigma$, and $\left(\phi_{1}\right)_{*}$ takes $\xi_{\alpha_{0}}$ to $\xi_{\alpha_{1}}$. In other words, after a change of coordinates we may write

$$
\alpha_{0}=h_{0}(r, \theta, x)\left(\beta_{0}+r^{2} d \theta\right)
$$

on $U$, for some positive function $h_{0}: U \rightarrow \mathbb{R}$ and sufficiently small $\delta$. Note that we have not modified $\alpha_{0}$ by a conformal factor, and $R_{\alpha_{0}}$ still satisfies $(\pitchfork)$.

Now let $h: U \rightarrow \mathbb{R}$ be any positive function. We claim that the Reeb vector field $R_{\alpha}$ for the contact form $\alpha=h\left(\beta_{0}+r^{2} d \theta\right)$ is positively transverse to the surfaces $\{\theta=$ const $\} \subset$ $U-\Gamma$ if and only if $\partial h / \partial r<0$. Indeed, by plugging $R_{\alpha}$ into the equation $\alpha=$ $h\left(\beta_{0}+r^{2} d \theta\right)$, we obtain

$$
\beta_{0}\left(R_{\alpha}\right)=\frac{1}{h}-r^{2} d \theta\left(R_{\alpha}\right)
$$

Also, the coefficient of $d r$ in the equation $\iota_{R_{\alpha}} d \alpha=0$ gives

$$
\frac{\partial h}{\partial r} \beta_{0}\left(R_{\alpha}\right)+\left(r^{2} \frac{\partial h}{\partial r}+2 r h\right) d \theta\left(R_{\alpha}\right)=0 .
$$


Putting the two identities together, we obtain

$$
\frac{\partial h}{\partial r}=-2 r h^{2} d \theta\left(R_{\alpha}\right)
$$

and the conclusion follows.

Now we take a function $h$ on $U$ with the following properties:

- $h=h_{0}$ on $\partial U \cap\{r=\delta\}$.

- $\partial h / \partial r<0$.

- $h=C_{0} / r^{2}$ when $\frac{\varepsilon}{2} \leq r \leq \varepsilon$. (Here $C_{0}>0$ is a large constant and $\varepsilon>0$ is a small constant $<\delta$.)

If we define $\alpha$ to be $h\left(\beta_{0}+r^{2} d \theta\right)$ on $U$ and $\alpha_{0}$ on $M-U$, then the Reeb vector field $R_{\alpha}$ is transverse to $R_{ \pm}(\Gamma)$. On $\frac{\varepsilon}{2} \leq r \leq \varepsilon$, since $\alpha=\left(C_{0} / r^{2}\right) \beta_{0}+C_{0} d \theta$ we have $R_{\alpha}=\left(1 / C_{0}\right) \partial_{\theta}$. We then take $M^{\prime}=M-\left\{r<\frac{\varepsilon}{2}\right\}, \Gamma^{\prime}=\left\{r=\frac{\varepsilon}{2}, \theta=\frac{3 \pi}{2}\right\}$ and $U\left(\Gamma^{\prime}\right)=M \cap\left\{\frac{\varepsilon}{2} \leq r \leq \varepsilon\right\}$. The $\theta$-coordinate becomes the $t$-coordinate on $U\left(\Gamma^{\prime}\right)$ and the contact form $\alpha$ gives this modified manifold $\left(M^{\prime}, \Gamma^{\prime}, U\left(\Gamma^{\prime}\right)\right)$ the structure of a sutured contact manifold.

Finally, $\Gamma$ is isotopic to $\Gamma^{\prime}$ through contact submanifolds of type $\left(\Gamma_{a, b}, \operatorname{ker} \beta_{0}\right)$, where $\Gamma_{a, b}=\{r=a, \theta=b\}$.

\subsection{From concave to convex boundary}

Definition 4.2 Let $M$ be a compact $(2 n+1)$-dimensional manifold with $3 \pi / 2-$ corners and let $\Gamma \subset \partial M$ be a $(2 n-1)$-dimensional submanifold. We call $(M, \Gamma, V(\Gamma))$ a concave sutured manifold with suture $\Gamma$ if $V(\Gamma) \subset M$ is a neighborhood of $\Gamma=\{(0,0)\} \times \Gamma$ of the form

$$
V(\Gamma)=(([-1,1] \times[-2,2])-((0,1] \times(-1,1))) \times \Gamma
$$

such that

- $\quad V(\Gamma) \cap \partial M=((\{0\} \times[-1,1]) \cup([0,1] \times\{-1,1\})) \times \Gamma ;$

- $\partial M-(\{0\} \times(-1,1) \times \Gamma)$ is the disjoint union of two submanifolds $R_{+}(\Gamma)$ and $R_{-}(\Gamma)$, such that $R_{ \pm}(\Gamma) \cap V(\Gamma)=[0,1] \times\{\mp 1\} \times \Gamma$;

- the corners of $M$ are $\{0\} \times\{-1,1\} \times \Gamma$.

We denote the coordinates on $V(\Gamma)$ above by $(\tau, t, x)$. We designate $R_{+}(\Gamma) \sqcup R_{-}(\Gamma)$ as the "horizontal boundary" of $M$ and $\{0\} \times[-1,1] \times \Gamma$ as the "vertical boundary". We endow $R_{+}(\Gamma)$ (resp. $R_{-}(\Gamma)$ ) with the boundary orientation (resp. the opposite of the boundary orientation) of $\partial M$. 
Definition 4.3 $(M, \Gamma, V(\Gamma), \xi)$ is a concave sutured contact manifold if $\xi$ is a contact structure on $M$ and if there exists a contact form $\alpha$ for $\xi$ so that $\left(R_{ \pm}(\Gamma),\left.\alpha\right|_{R_{ \pm}(\Gamma)}\right)$ are Liouville manifolds and $\alpha=C d t+\beta$ in $V(\Gamma)$, where $C>0$ and $\beta$ is independent of $t$ and has no $d t$-term. (Note that the Reeb flow along the vertical boundary then goes from $R_{+}(\Gamma)$ to $R_{-}(\Gamma)$, instead of from $R_{-}(\Gamma)$ to $R_{+}(\Gamma)$ as is the case for convex sutures).

Example 4.4 Let $(M, \xi=\operatorname{ker} \alpha)$ be a contact 3-manifold and let $S \subset M$ be a compact, oriented surface which is transverse to the Reeb vector field $R_{\alpha}$ and whose boundary $\partial S$ is positively transverse to $\xi$. Now, if $N(S)=S \times[-\varepsilon, \varepsilon]$ is a collar neighborhood of $S$ whose $[-\varepsilon, \varepsilon]$-coordinate $t$ satisfies $R_{\alpha}=\partial_{t}$, then $M-\operatorname{int}(N(S))$ is naturally a concave sutured contact manifold with respect to the form $\alpha$. In particular, $\Gamma=\{0\} \times \partial S$, the vertical boundary is $\partial S \times[-\varepsilon, \varepsilon], R_{+}(\Gamma)=S \times\{-\varepsilon\}$, and $R_{-}(\Gamma)=$ $S \times\{+\varepsilon\}$.

Example 4.5 Let $(M, \xi)$ be a closed $(2 n+1)$-dimensional contact manifold and let $L \subset M$ be a closed Legendrian submanifold. By the Darboux-Weinstein neighborhood theorem, there is a sufficiently small neighborhood $N(L)$ of $L$ which is contactomorphic to a small neighborhood of the zero section $\left\{z=p_{1}=\cdots=p_{n}=0\right\}$ in the 1 -jet space $\mathbb{R} \times T^{*} L$ with the contact 1 -form $\alpha=d z+\lambda$, where $\lambda$ is the Liouville form on $T^{*} L$ which is locally given by $\sum_{i} p_{i} d q_{i}$. The Reeb vector field is given by $R_{\alpha}=\partial_{z}$, and we can take the boundary of the tubular neighborhood of the zero section to be $\Sigma=\left\{\left.(z, p, q)\left|z^{2}+\right| p\right|_{q} ^{2}=\varepsilon^{2}\right\}$, where $|\cdot|_{q}$ denotes some fiber metric on $T_{q}^{*} L$ which is smooth in $q \in L$. Then $R_{\alpha}$ is positively transverse to $\Sigma$ (with the boundary orientation) for $z>0$, negatively transverse to $\Sigma$ for $z<0$, and tangent to $\Sigma$ for $z=0$. The set $\Gamma=\left\{\left.(z, p, q)|z=0| p\right|_{q}=,\varepsilon\right\}$ is the unit cotangent bundle of $L$, and is a $(2 n-1)$-dimensional contact manifold. One can see this for example by observing that the Liouville vector field $\sum_{i} p_{i} \partial_{p_{i}}$ for $\left(T^{*} L, \lambda\right)$ is transverse to $\Gamma$. If we define a new neighborhood of $L$ by $N(L)=\left\{\left.(z, p, q)\left|z^{2}<\varepsilon^{2},\right| p\right|_{q} ^{2}<\varepsilon^{2}\right\}$, then $\left(M-N(L),\left.\xi\right|_{M-N(L)}, \Gamma\right)$ is a concave sutured manifold.

Proposition 4.6 Let $\mathcal{M}=(M, \Gamma, V(\Gamma), \xi)$ be a concave sutured contact manifold. Then there is an inclusion of $\mathcal{M}$ into a convex sutured contact manifold $\mathcal{M}^{\prime}=$ $\left(M^{\prime}, \Gamma^{\prime}, U\left(\Gamma^{\prime}\right), \xi^{\prime}\right)$, so that the contact manifold with convex boundary $\left(M_{\mathrm{sm}}, \Gamma, \xi\right)$, obtained by smoothing the corners of $\mathcal{M}$, is isotopic to the contact manifold with convex boundary $\left(M_{\mathrm{sm}}^{\prime}, \Gamma^{\prime}, \xi^{\prime}\right)$, obtained by smoothing the corners of $\mathcal{M}^{\prime}$. Here $\Gamma$ and $\Gamma^{\prime}$ are isotopic contact submanifolds and $M^{\prime}-M \subset(0,1] \times(-1,1) \times \Gamma$.

Proof On $V(\Gamma)=([-1,1] \times[-2,2]-(0,1] \times(-1,1)) \times \Gamma$ the adapted contact form is $\alpha=C d t+\beta$, where $C$ is a positive constant. Without loss of generality we can 
write $\beta=e^{-\tau} \beta_{0}$, where $\beta_{0}$ is a 1 -form on $\Gamma$. (The minus sign in $e^{-\tau} \beta_{0}$ is due to the fact that the Liouville vector field on $R_{ \pm}(\Gamma)$ points in the negative $\tau$-direction.) We now describe how to extend $\alpha$ to the product $[0,1] \times[-1,1] \times \Gamma$. To that end, we look for a form of type $f(\tau, t) d t+g(\tau, t) \beta_{0}$, where $f, g:[-1,1] \times[-2,2] \rightarrow \mathbb{R}$, and $f=C$ and $g=e^{-\tau}$ outside of $[0,1] \times[-1,1]$.

Let $g$ be a positive Morse function on $[0,1] \times[-1,1]$ which is a $C^{0}$-small perturbation of $e^{-\tau}$ and whose level sets are obtained from perturbing the foliation by intervals $\{\tau\} \times[-1,1], \tau \in[0,1]$, by adding a pair of (canceling) critical points - a saddle $h$ and a source $e-$ as in Figure 1.Two of the separatrices of $h$ go to $[0,1] \times\{ \pm 1\}$ and

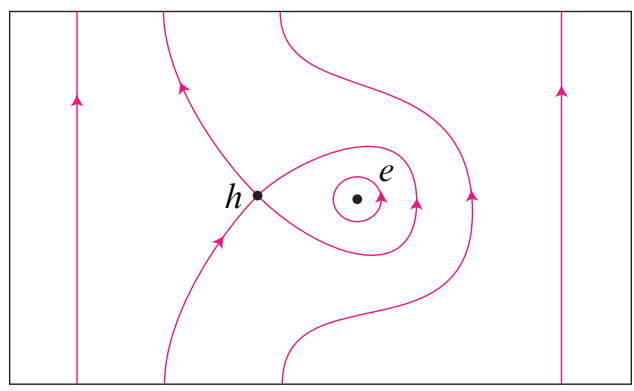

Figure 1: The level sets of $g$ on $[0,1] \times[-1,1]$. The arrows indicate the direction of $X_{g}$.

decompose $[0,1] \times[-1,1]$ into two components; we assume that $e$ is on the component which contains $(1,0)$. We choose $g$ so that $\partial g / \partial \tau<\varepsilon$, where $\varepsilon$ is a small positive constant. (Here $\partial g / \partial \tau$ may be negative.) Note that $\partial g / \partial \tau>0$ (resp. $<0$ ) at those points in Figure 1 where the arrows on the level sets point downwards (resp. upwards). Next choose a positive function $f$ on $[0,1] \times[-1,1]$ so that $\partial f / \partial \tau \geq 0$ on $[0,1] \times[-1,1]$ and $\partial f / \partial \tau$ is a large positive constant where $\partial g / \partial \tau \geq 0$.

On $[0,1] \times[-1,1] \times \Gamma$, with $\alpha$ defined as above, we compute

$$
d \alpha=\frac{\partial f}{\partial \tau} d \tau \wedge d t+d g \wedge \beta_{0}+g d \beta_{0} .
$$

The contact condition for $\alpha$ is

$$
g^{n-1}\left(g \frac{\partial f}{\partial \tau}-f \frac{\partial g}{\partial \tau}\right)>0,
$$

and the requirements on $\partial f / \partial \tau$ and $\partial g / \partial \tau$ yield the contact condition.

Let $X_{g}$ be the Hamiltonian vector field with respect to the symplectic form $d \tau \wedge d t$. Note that $X_{g}$ is tangent to the level sets of $g$. The Reeb vector field $R_{\alpha}$ is parallel 
to $(\partial f / \partial \tau) R_{0}+X_{g}$, where $R_{0}$ is the Reeb vector field for $\beta_{0}$ on $\Gamma$. Indeed, we compute that

$$
\begin{aligned}
l_{(\partial f / \partial \tau) R_{0}+X_{g} d \alpha} & =\frac{\partial f}{\partial \tau} \cdot l_{X_{g}}(d \tau \wedge d t)+l_{(\partial f / \partial \tau) R_{0}}\left(d g \wedge \beta_{0}\right)+g \cdot l_{(\partial f / \partial \tau) R_{0}} d \beta_{0} \\
& =\frac{\partial f}{\partial \tau} d g-\frac{\partial f}{\partial \tau} d g+0=0 .
\end{aligned}
$$

Let $\delta$ be an arc in $[0,1] \times[-1,1]$ which connects the source $e$ to the point $(1,0)$ and is transverse to $X_{g}$. Let $D$ be a small disk of radius $r$ about $e$, whose boundary is a level set of $g$, and let $N_{\varepsilon}$ be an $\varepsilon$-neighborhood of $\delta$, with $\varepsilon \ll r$. Consider the manifold $M^{\prime \prime}$, obtained from $M$ by adding $\left([0,1] \times[-1,1]-\operatorname{int}\left(D \cup N_{\varepsilon}\right)\right) \times \Gamma$. See Figure 2. The contact form on $M^{\prime \prime}$ is the restriction of $\alpha$, defined above. We then

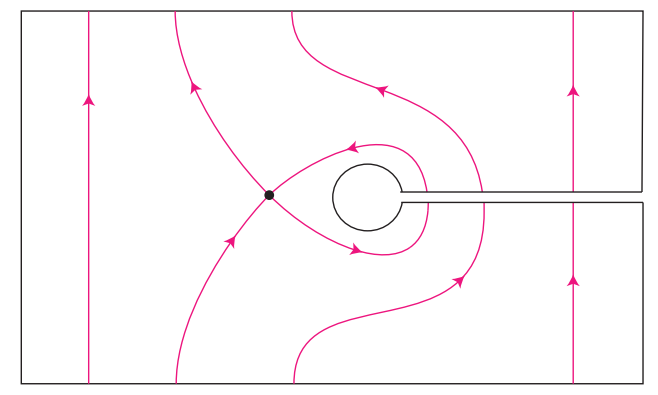

Figure 2: Excavation of $D \cup N_{\varepsilon}$ from $[0,1] \times[-1,1]$

modify $M^{\prime \prime}$ slightly so that the corners along $\tau=1$ are smoothed and the horizontal boundary is transverse to $R_{\alpha}$. Note that $R_{\alpha}$ is tangent to $\left(\partial D-N_{\varepsilon}\right) \times \Gamma$ and the orbits connect from $R_{-}(\Gamma)$ to $R_{+}(\Gamma)$; we may also need to make a slight modification so that the flow lines of the Reeb vector field from $R_{-}(\Gamma)$ to $R_{+}(\Gamma)$ have constant length near the vertical boundary. The resulting manifold $\left(M^{\prime},\left.\alpha\right|_{M^{\prime}}\right)$ is a (convex) sutured contact manifold whose vertical boundary contains $\left(\partial D-N_{\varepsilon}\right) \times \Gamma$.

Finally, the isotopy of $\left(M_{\mathrm{sm}}, \Gamma, \xi\right)$ to $\left(M_{\mathrm{sm}}^{\prime}, \Gamma^{\prime}, \xi^{\prime}\right)$ follows from observing that there is a 1-parameter family of convex submanifolds which connect between $\partial M_{\mathrm{sm}}$ and $\partial M_{\mathrm{sm}}^{\prime}$ inside $M$. We use Lemma 2.2 and find submanifolds which are (positively or negatively) transverse to $R_{\alpha}$ except at some contact submanifold $\{(\tau, t)\} \times \Gamma$, where $(\tau, t) \in$ $[0,1] \times[-1,1]$.

The only periodic orbits of $R_{\alpha}$ that are contained in $M^{\prime}-M$ are periodic orbits of $R_{0}$ contained in $\{h\} \times \Gamma$. When $\operatorname{dim} M=3$, this construction gives a collection of hyperbolic orbits (one for each component of $\Gamma$ ) which are parallel to the suture $\Gamma$. 


\subsection{Gluing sutured contact manifolds}

The procedure of gluing sutured contact manifolds, together with compatible Reeb vector fields, was first described by Colin and Honda [8] when $\operatorname{dim} M=3$. Here we describe the sutured gluing so that it is also applicable to higher dimensions.

Let $\left(M^{\prime}, \Gamma^{\prime}, U\left(\Gamma^{\prime}\right), \xi^{\prime}\right)$ be a sutured contact manifold of dimension $2 m+1$ and let $\alpha^{\prime}$ be an adapted contact form. Let

$$
\pi: U\left(\Gamma^{\prime}\right)=[-1,0] \times[-1,1] \times \Gamma^{\prime} \rightarrow[-1,0] \times \Gamma^{\prime},
$$

be the projection onto the first and third factors. If we think of $[-1,0] \times \Gamma^{\prime}$ as a subset of $R_{+}\left(\Gamma^{\prime}\right)$ (resp. $R_{-}\left(\Gamma^{\prime}\right)$ ), then we denote the projection by $\pi_{+}$(resp. $\pi_{-}$). By definition, the horizontal components $\left(R_{ \pm}\left(\Gamma^{\prime}\right), \beta_{ \pm}^{\prime}=\left.\alpha^{\prime}\right|_{R_{ \pm}}\left(\Gamma^{\prime}\right)\right)$ are Liouville manifolds. We denote by $Y_{ \pm}^{\prime}$ their Liouville vector fields. The contact form $\alpha^{\prime}$ is $d t+\beta_{ \pm}^{\prime}$ on the neighborhoods $R_{+}\left(\Gamma^{\prime}\right) \times[1-\varepsilon, 1]$ and $R_{-}\left(\Gamma^{\prime}\right) \times[-1,-1+\varepsilon]$ of $R_{+}\left(\Gamma^{\prime}\right)=R_{+}\left(\Gamma^{\prime}\right) \times\{1\}$ and $R_{-}\left(\Gamma^{\prime}\right)=R_{-}\left(\Gamma^{\prime}\right) \times\{-1\}$, found using the Reeb flow. Also, we may assume that the Reeb vector field $R_{\alpha^{\prime}}$ is given by $\partial_{t}$ on $U\left(\Gamma^{\prime}\right)$, after scaling the contact form.

Take a $2 m$-dimensional submanifold $P_{+} \subset R_{+}\left(\Gamma^{\prime}\right)$ with smooth boundary ${ }^{5}$ so that

- $\partial P_{+}$is the union of $\left(\partial P_{+}\right)_{\partial} \subset \partial R_{+}\left(\Gamma^{\prime}\right)$ and $\left(\partial P_{+}\right)_{\text {int }} \subset \operatorname{int}\left(R_{+}\left(\Gamma^{\prime}\right)\right)$;

- $\partial P_{+}$is positively transverse to the Liouville vector field $Y_{+}^{\prime}$ on $R_{+}\left(\Gamma^{\prime}\right)$.

Similarly take $P_{-} \subset R_{-}\left(\Gamma^{\prime}\right),\left(\partial P_{-}\right)_{\partial}$, and $\left(\partial P_{-}\right)_{\text {int }}$ with $Y_{-}^{\prime}$ positively transverse to $\partial P_{-}$. See Figure 3 . Whenever we refer to $\left(\partial P_{ \pm}\right)_{\text {int }}$ and $\left(\partial P_{ \pm}\right)_{\partial}$, we assume that closures are taken as appropriate.

Suppose we have a pair $P_{+}, P_{-}$so that $\pi\left(\left(\partial P_{-}\right)_{\partial}\right) \cap \pi\left(\left(\partial P_{+}\right)_{\partial}\right)=\varnothing$ and there is a diffeomorphism $\phi$ which sends $\left(P_{+}, \beta_{+}^{\prime} \mid P_{+}\right)$to $\left(P_{-},\left.\beta_{-}^{\prime}\right|_{P_{-}}\right)$and takes $\left(\partial P_{+}\right)_{\text {int }}$ to $\left(\partial P_{-}\right)_{\partial}$ and $\left(\partial P_{+}\right)_{\partial}$ to $\left(\partial P_{-}\right)_{\text {int }}$. We will refer to the triple $\left(P_{+}, P_{-}, \phi\right)$ as the gluing data. For the purposes of gluing, it suffices to require that $\left.\beta_{+}^{\prime}\right|_{P_{+}}$and $\phi^{*}\left(\left.\beta_{-}^{\prime}\right|_{P_{-}}\right)$ be homotopic Liouville 1 -forms on $P_{+}$, via a homotopy which is constant in a neighborhood of $\partial P_{+}$. In that case, there is a 1-parameter family of adapted contact 1forms $\left(\alpha^{\prime}\right)^{\sigma}, \sigma \in[0,1]$, on $\left(M^{\prime}, \Gamma^{\prime}, U\left(\Gamma^{\prime}\right)\right)$ so that $\left(\alpha^{\prime}\right)^{0}=\alpha^{\prime},\left(\alpha^{\prime}\right)^{\sigma}=C^{\sigma} d t+\left(\beta^{\prime}\right)_{ \pm}^{\sigma}$ on $R_{ \pm}\left(\Gamma^{\prime}\right),\left(\beta^{\prime}\right)_{ \pm}^{\sigma}=\beta_{ \pm}^{\prime}$ on $R_{ \pm}\left(\Gamma^{\prime}\right)-\operatorname{int}\left(P_{ \pm}\right)$, and $\left.\left(\beta^{\prime}\right)_{+}^{1}\right|_{P_{+}}=\phi^{*}\left(\left.\left(\beta^{\prime}\right)_{-}^{1}\right|_{P_{-}}\right)$. This is made possible by the flexibility theorem of Giroux [15]. (Note that, when $\operatorname{dim} M^{\prime}=3$, we only need $\left.\beta_{+}^{\prime}\right|_{P_{+}}$and $\phi^{*}\left(\left.\beta_{-}^{\prime}\right|_{P_{-}}\right)$to match up on $\partial P_{+}$, since we can linearly interpolate between primitives of positive area forms on a surface.)

\footnotetext{
${ }^{5}$ This is slightly different from what appears in [8], where it is assumed that $\partial P_{+}$has corners along $\partial\left(\partial P_{+}\right)_{\partial}=\partial\left(\partial P_{+}\right)_{\text {int }}$.
} 


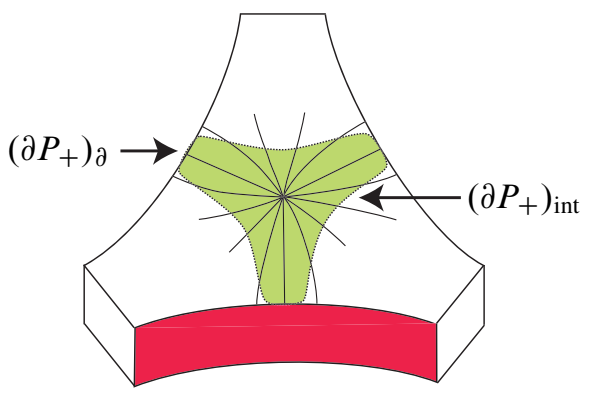

Figure 3: The diagram shows $P_{+} \subset R_{+}\left(\Gamma^{\prime}\right)$. The line field represents $Y_{+}^{\prime} \subset \operatorname{ker} \beta_{+}^{\prime}$, and the vertical annuli represent the vertical boundary of $M^{\prime}$.

Topologically, we construct the sutured manifold $(M, \Gamma)$ from $\left(M^{\prime}, \Gamma^{\prime}\right)$ and the gluing data $\left(P_{+}, P_{-}, \phi\right)$ as follows: Let $M=M^{\prime} / \sim$, where

(1) $x \sim \phi(x)$ for all $x \in P_{+}$;

(2) $x \sim x^{\prime}$ if $x, x^{\prime} \in \pi^{-1}\left(\Gamma^{\prime}\right)$ and $\pi(x)=\pi\left(x^{\prime}\right) \in \Gamma^{\prime}$.

In words, (2) says that we collapse the annular neighborhood of $\Gamma^{\prime}$ onto $\Gamma^{\prime}$. Then

$$
\begin{array}{cl}
R_{+}(\Gamma)=\overline{\left(R_{+}\left(\Gamma^{\prime}\right)-P_{+}\right)} / \sim, & \text { ie }\left(\partial P_{+}\right)_{\text {int }} \text { is identified with } \pi_{+}\left(\left(\partial P_{-}\right)_{\partial}\right), \\
R_{-}(\Gamma)=\overline{\left(R_{-}\left(\Gamma^{\prime}\right)-P_{-}\right)} / \sim, \quad \text { ie }\left(\partial P_{-}\right)_{\text {int }} \text { is identified with } \pi_{-}\left(\left(\partial P_{+}\right)_{\partial}\right),
\end{array}
$$

and

$$
\Gamma=\overline{\left(\Gamma^{\prime}-\pi\left(\partial P_{+} \sqcup \partial P_{-}\right)\right)} / \sim .
$$

As it is defined, the boundary of $(M, \Gamma)$ has a cusp along $\Gamma$. To obtain an actual sutured manifold, one "blows up" the cusp $\Gamma$ to a vertical annulus $[-1,1] \times \Gamma$.

In [14, Definition 3.1], Gabai defined the notion of a sutured manifold decomposition for sutured 3-manifolds, which is the inverse construction of our sutured gluing.

Fact 4.7 Suppose $\operatorname{dim} M=3$. Let $P \subset(M, \Gamma)$ be the surface obtained by identifying $P_{+}$and $P_{-}$. Then $P$ gives rise to a sutured manifold decomposition

$$
(M, \Gamma) \stackrel{P}{\rightarrow}\left(M^{\prime}, \Gamma^{\prime}\right) .
$$

Construction of $\left(M_{n}, \alpha_{n}\right)$ For the purposes of studying holomorphic curves, we want to stretch in both the $\tau$ - and $t$-directions. The construction of the contact manifold will depend on the parameter $n$, and the resulting glued-up sutured contact manifold will be written as

$$
\left(M_{n}, \Gamma_{n}, U\left(\Gamma_{n}\right), \xi_{n}=\operatorname{ker}\left(\alpha_{n}\right)\right)
$$

See Figure 4 for an illustration when $n=1$. 


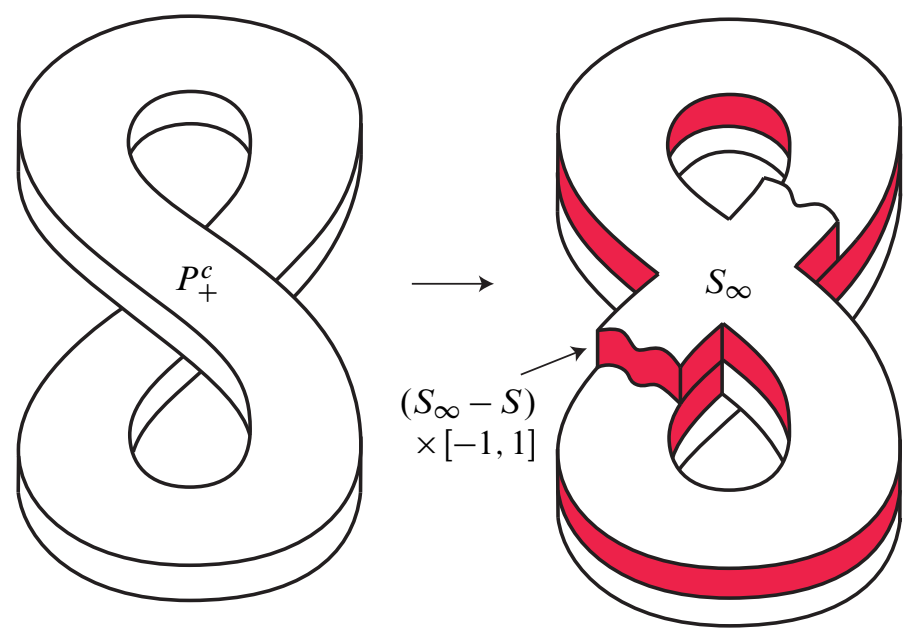

Figure 4: The left-hand side represents $M^{\prime} / \sim$, where $x \sim \phi(x)$ for all $x \in P_{+}$. The portion of $R_{+}\left(\Gamma^{\prime}\right)$ that is exposed is $P_{+}^{c}$. The right-hand side represents $M^{\prime} / \sim$ with $\left(S_{\infty}-S\right) \times[-1,1]$ glued. When $n=1$ this gives us $M^{(1)}$.

Step 1: Gluing the top and the bottom Let $\left(M^{(0)}, \alpha^{(0)}\right)=\left(M_{n}^{(0)}, \alpha_{n}^{(0)}\right)$ - we will often suppress $n$ to avoid cluttering the notation - be the contact manifold obtained from the completion $\left(\left(M^{\prime}\right)^{*},\left(\alpha^{\prime}\right)^{*}\right)$ by removing the Side, ie $M^{(0)}=$ $M^{\prime} \cup\left(R_{+}\left(\Gamma^{\prime}\right) \times[1, \infty)\right) \cup\left(R_{-}\left(\Gamma^{\prime}\right) \times(-\infty,-1]\right)$. Then construct $\left(M^{(1)}, \alpha^{(1)}\right)$ from

$$
M^{(0)}-\left(P_{+} \times[n, \infty)\right)-\left(P_{-} \times(-\infty,-n]\right),
$$

by taking closures and identifying

- $P_{+} \times\{n\}$ with $P_{-} \times\{-n\}$;

- $\left(\partial P_{+}\right)_{\text {int }} \times[n, \infty)$ with $\left(\partial P_{-}\right)_{\partial} \times[-n, \infty)$;

- $\left(\partial P_{+}\right)_{\partial} \times(-\infty, n]$ with $\left(\partial P_{-}\right)_{\text {int }} \times(-\infty,-n]$;

all via the identification $(x, t) \mapsto(\phi(x), t-2 n)$. Let us write $P_{+}^{c}=\overline{R_{+}\left(\Gamma^{\prime}\right)-P_{+}}$ and $P_{-}^{c}=\overline{R_{-}\left(\Gamma^{\prime}\right)-P_{-}}$. Next take $n^{\prime} \gg 0$ and truncate the Top and Bottom of $\left(M^{(1)}, \alpha^{(1)}\right)$ to obtain the (compact) sutured manifold $\left(M^{(2)}, \Gamma^{(2)}, U\left(\Gamma^{(2)}\right)\right)$ with contact form $\alpha^{(2)}$ so that $M^{(2)}$ contains

$$
M^{\prime} \cup\left(P_{+}^{c} \times\left[1, n^{\prime}\right]\right) \cup\left(P_{-}^{c} \times\left[-n^{\prime},-1\right]\right),
$$

the Reeb vector field $R=R_{\alpha^{(2)}}$ is transverse to the horizontal boundary, and the vertical boundary $E$ is foliated by interval orbits of $R$ with fixed action $\geq 3 n^{\prime}$. 
Step 2: Extending the side Let $\rho: E \rightarrow B$ be the fibration whose fibers are the interval orbits of $R$, so that $B$ is diffeomorphic to $\Gamma$. The base $B$ is a union of finitely many codimension zero submanifolds $B_{i}$ so that there are local sections $s_{i}: B_{i} \rightarrow \rho^{-1}\left(B_{i}\right)$ for which $s_{i}\left(B_{i}\right)$ are $(2 n-1)$-dimensional contact submanifolds. Let $(x, t)$ be coordinates on $\rho^{-1}\left(B_{i}\right)$ so that $R=\partial_{t}, x$ is a local coordinate system for $B_{i}$, and $t=0$ corresponds to $s_{i}\left(B_{i}\right)$. We consider the extension

$$
\tilde{\rho}:[0, \infty) \times E \rightarrow[0, \infty) \times B
$$

with first coordinate $\tau$ so that $\{0\} \times E$ is identified with $E \subset M^{(2)}$ and $\widetilde{\rho}(\tau, x, t)=$ $(\tau, \rho(x, t))$. We can extend the contact form $\alpha^{(2)}$ to a $t$-invariant contact form on $[0, \infty) \times E$ which is given by $d t+e^{\tau} \beta_{0}(x)$, where $(\tau, x, t)$ are coordinates on $[0, \infty) \times \rho^{-1}\left(B_{i}\right)$.

At this point we are not guaranteed the existence of a global section $s: B \rightarrow E$ which is contact when $\tau=0$. However, given any section $s: B \rightarrow E$, for sufficiently large $\tau=\tau_{0}$, we claim that the submanifold $\left\{\tau_{0}\right\} \times s(B)$ is contact. Indeed, any section $s$ can locally be written as $(x, t) \mapsto(x, f(x))$, and pulling back $d t+e^{\tau} \beta_{0}(x)$ yields $d f(x)+e^{\tau} \beta_{0}(x)$. If $\tau_{0} \gg 0$, the term $e^{\tau} \beta_{0}(x)$ dominates $d f(x)$, and the section becomes contact. Attaching

$$
V=\left[0, \tau_{0}\right] \times E
$$

to $M^{(2)}$ gives us $\left(M_{n}, \alpha_{n}\right)$. The horizontal boundary which is positively (resp. negatively) transverse to $R$ will be called $R_{+}\left(\Gamma_{n}\right)$ (resp. $R_{-}\left(\Gamma_{n}\right)$ ).

We now verify that $R_{ \pm}\left(\Gamma_{n}\right)$ are Liouville manifolds. The 1 -form $\alpha_{n}$ restricts to the primitive of a symplectic form on $R_{ \pm}\left(\Gamma_{n}\right)$, since $R$ is transverse to $R_{ \pm}\left(\Gamma_{n}\right)$. Without loss of generality the ends of $R_{ \pm}\left(\Gamma_{n}\right)$ are of the form $\left[0, \tau_{0}\right] \times \partial E$ with local contact form $d t+d f(x)+e^{\tau} \beta_{0}(x)$. As before, when $\tau_{0} \gg 0, e^{\tau} \beta_{0}(x)$ dominates $d f(x)$, and the Liouville vector field corresponding to $d f(x)+e^{\tau} \beta_{0}(x)$ approaches one parallel to $\partial_{\tau}$. It now follows that the resulting manifold $\left(M_{n}, \Gamma_{n}, U\left(\Gamma_{n}\right), \xi_{n}, \alpha_{n}\right)$ is a sutured contact manifold.

Now we describe the completion $M_{n}^{*}$ of $M_{n}$. Let

$$
V^{*}=[0, \infty) \times \mathbb{R} \times B
$$

be the completion of $V=\left[0, \tau_{0}\right] \times E$, obtained by extending to (T), (B), and (S). Then $M_{n}^{*}$ is obtained from $M^{(1)}$ by attaching $V^{*}$.

Step 3: Interval-fibered extension Let

$$
S=\left(R_{+}\left(\Gamma^{\prime}\right) \times\{n\}\right) \cup\left(R_{-}\left(\Gamma^{\prime}\right) \times\{-n\}\right) \subset M^{(1)},
$$


and let $S_{\infty} \subset M^{(1)}$ be the noncompact, possibly disconnected surface obtained from $S$ by attaching all the $P_{+}^{c} \times\{(2 k+1) n\}$ and $P_{-}^{c} \times\{(-2 k-1) n\}$, where $k$ ranges over all the positive integers. Note that it is possible for $S_{\infty}$ to have finitely many noncompact components and countably many compact components. There is an embedding

$$
\eta_{n}: S_{\infty} \times[-n+1, n-1] \hookrightarrow M^{(1)},
$$

which maps $(x, t)$ to the time- $t$ translation of $x \in S_{\infty}$ along $\partial_{t}$, such that

$$
M_{e}^{\prime}=M^{(1)}-\left(S_{\infty} \times[-n+1, n-1]\right)
$$

is obtained from $M^{\prime}$ by attaching an interval-fibered product which is diffeomorphic to $\left(S_{\infty}-S\right) \times[-1,1]$.

We will call $M_{e}^{\prime}$ an infinite interval-fibered extension (ie an exhaustion of intervalfibered extensions) of $\left(M^{\prime}, \Gamma^{\prime}\right)$. More explicitly,

$$
M_{e}^{\prime}=M^{\prime} \cup \bigcup_{k>0}\left(P_{+}^{c} \times[2 k n-1,2 k n+1]\right) \cup \bigcup_{k>0}\left(P_{-}^{c} \times[-2 k n-1,-2 k n+1]\right),
$$

where the gluing maps are given as before by $(x, t) \mapsto(\phi(x), t-2 n)$ for $x \in P_{+}$.

We can write $\overline{S_{\infty} \backslash S}=S_{+} \cup S_{-}$, where $S_{+}$is the subsurface obtained by gluing together the $P_{+}^{c} \times\{(2 k+1) n\}$ pieces and $S_{-}$is the subsurface obtained by gluing together the $P_{-}^{c} \times\{(-2 k-1) n\}$ pieces. Let us denote by $\left(\partial P_{+}\right)_{\text {int }} \subset S_{+}$the union of connected components of $\left(\partial P_{+}\right)_{\text {int }} \times\{(2 k+1) n\}$ which are on the boundary of $S_{+}$, ie when $k=1$; similarly define $\left(\partial P_{-}\right)_{\text {int }} \subset S_{-}$. Then we can write $M_{e}^{\prime}$ more abstractly as

$$
M^{\prime} \cup\left(S_{-} \times[-1,1]\right) \cup\left(S_{+} \times[-1,1]\right),
$$

where we glue $\left(\partial P_{+}\right)_{\text {int }} \times[-1,1] \subset S_{+} \times[-1,1]$ to $\left(\partial P_{-}\right)_{\partial} \times[-1,1] \subset M^{\prime}$ by $(\phi, i d)$, and $\left(\partial P_{-}\right)_{\text {int }} \times[-1,1] \subset S_{-} \times[-1,1]$ to $\left(\partial P_{+}\right)_{\partial} \times[-1,1] \subset M^{\prime}$.

Summarizing, we have the following:

Lemma 4.8 Suppose $n>0$. Given a sutured contact manifold $\left(M^{\prime}, \Gamma^{\prime}, U\left(\Gamma^{\prime}\right), \alpha^{\prime}\right)$ and gluing data $\left(P_{+}, P_{-}, \phi\right)$, there exists an inclusion of sutured contact manifolds

$$
\left(M^{\prime}, \Gamma^{\prime}, U\left(\Gamma^{\prime}\right), \alpha^{\prime}\right) \hookrightarrow\left(M_{n}, \Gamma_{n}, U\left(\Gamma_{n}\right), \alpha_{n}\right),
$$

where $\left(M_{n}, \Gamma_{n}, U\left(\Gamma_{n}\right)\right)$ is homeomorphic to $(M, \Gamma, U(\Gamma))$ and the completion of $M_{n}$ is $M_{n}^{*}=M^{(1)} \cup V^{*}$. Here $V^{*}$ is a fibered piece given by Equation (11) and $M^{(1)}$ admits a noncompact embedding of $S_{\infty} \times[-n+1, n-1]$ so that $R_{\alpha_{n}}=\partial_{t}$ on $\left(S_{\infty}-S\right) \times[-n+1, n-1]$ and $S \times([-n+1,-\varepsilon] \cup[\varepsilon, n-1])$, and the complement $M^{(1)}-\left(S_{\infty} \times[-n+1, n-1]\right)$ is an infinite interval-fibered extension of $\left(M^{\prime}, \Gamma^{\prime}, U\left(\Gamma^{\prime}\right), \alpha^{\prime}\right)$ which is independent of $n$. 
Almost complex structures We now discuss the gluing/extension of almost complex structures under sutured manifold gluing.

Step 1: Definition on $\mathbb{R} \times M^{\prime}$ Definition on $\mathbb{R} \times M^{\prime}$ We first define an almost complex structure $J_{\kappa}^{\prime}$ on $\mathbb{R} \times M^{\prime}$ which is tailored to the sutured contact manifold $\left(M^{\prime}, \Gamma^{\prime}, U\left(\Gamma^{\prime}\right), \alpha^{\prime}\right)$.

Consider the neighborhood $U\left(\Gamma^{\prime}\right)=[-1,0] \times[-1,1] \times \Gamma^{\prime}$ with coordinates $(\tau, t, x)$, where we may assume that $\beta_{+}^{\prime}=e^{\tau} \bar{\beta}_{0}$ and $\bar{\beta}_{0}=\left.\beta_{+}^{\prime}\right|_{\{0,0\} \times \Gamma^{\prime}}$. Choose a diffeomorphism

$$
\begin{aligned}
H_{\kappa}:[-1,0] \times \Gamma^{\prime} \stackrel{\sim}{\rightarrow}[0, \kappa] \times \Gamma^{\prime}, \\
(\tau, x) \mapsto\left(h_{\kappa}(\tau), x\right),
\end{aligned}
$$

where $h_{\kappa}:[-1,0] \stackrel{\sim}{\rightarrow}[0, \kappa], h_{\kappa}(-1)=0, h_{\kappa}(0)=\kappa, h_{\kappa}^{\prime}(\tau)=1$ in a neighborhood of $\tau=-1,0$, and $h_{\kappa}$ is linear outside a bigger neighborhood of $\tau=-1,0$.

Then $J_{\kappa}^{\prime}$ is defined on $\mathbb{R} \times U\left(\Gamma^{\prime}\right)$ by specifying the projection $\left(J_{\kappa}^{\prime}\right)_{0}$ to $T([-1,0] \times \Gamma)$ as follows:

- $\left(J_{\kappa}^{\prime}\right)_{0}$ is the $H_{\kappa}$-pullback of an almost complex structure adapted to $\bar{\beta}_{0}$ on $[0, \kappa] \times \Gamma^{\prime}$.

- $\phi_{*}$ takes $\left(J_{\kappa}^{\prime}\right)_{0}$ along $\partial\left(\partial P_{+}\right)_{\text {int }}$ to $\left(J_{\kappa}^{\prime}\right)_{0}$ along $\partial\left(\partial P_{-}\right)_{\text {int }}$, so that they agree when projected to the base $B$ of the fibration $\rho: E \rightarrow B$.

On $\mathbb{R} \times\left(M^{\prime}-U\left(\Gamma^{\prime}\right)\right)$, choose $J_{\kappa}^{\prime}$ to be independent of $\kappa$, such that the projection $\left(J_{\kappa}^{\prime}\right)_{0}$ to $T\left(R_{ \pm}\left(\Gamma^{\prime}\right)-\left([-1,0] \times \Gamma^{\prime}\right)\right)$ is adapted to $\beta_{ \pm}^{\prime}$.

Step 2: Definition on $\mathbb{R} \times M^{(1)}$ We then extend $J_{\kappa}^{\prime}$ to an almost complex structure $J_{\kappa, n}$ on $\mathbb{R} \times M^{(1)}$ which satisfies the following:

(1) $J_{\kappa, n}$ is adapted to the symplectization $\left(\mathbb{R} \times M^{(1)}, d\left(e^{s} \alpha^{(1)}\right)\right)$.

(2) $J_{\kappa, n}$ is $\partial_{t}$-invariant on each connected component of $\mathbb{R}$ times $P_{+}^{c} \times[2 n-1, \infty)$, $P_{-}^{c} \times(-\infty,-2 n+1], \partial M^{(1)}$, and $S \times([-n+1,-\varepsilon] \cup[\varepsilon, n-1])$.

(3) The extension to the interior of $S \times[-\varepsilon, \varepsilon]$ is arbitrary, but is independent of $n$.

Here $S \times[-n+1, n+1]$ is viewed as a subset of $M^{(1)}$ via the map $\eta_{n}$ given in Equation (12). The almost complex structure $J_{\kappa, n}$ on $\mathbb{R} \times P_{+}^{c} \times[2 n-1, \infty)$ is defined by specifying the projection $\left(J_{\kappa, n}\right)_{0}$ of $J_{\kappa, n}$ to $T P_{+}^{c}$ so that $\left(J_{\kappa, n}\right)_{0}$ agrees with $\left(J_{\kappa}^{\prime}\right)_{0}$ along $\partial P_{+}^{c}-\partial P_{+}$and with $\phi_{*}\left(J_{\kappa}^{\prime}\right)_{0}$ along $\partial P_{+}^{c} \cap \partial P_{+}=\left(\partial P_{+}\right)_{\text {int }}$. The extension of $\left(J_{\kappa, n}\right)_{0}$ to the interior of $P_{+}^{c}$ is arbitrary, provided it is compatible with $d \beta_{+}^{\prime}$. In 
particular, $\left(J_{\kappa, n}\right)_{0}$ does not need to agree with $\left(J_{\kappa}^{\prime}\right)_{0}$ on all of $P_{+}^{c}$. The almost complex structure $\left(J_{\kappa, n}\right)_{0}$ is defined similarly on $P_{-}^{c}$.

Step 3: Extension to $\mathbb{R} \times V^{*} \quad$ Next we extend $J_{\kappa, n}$ to $\mathbb{R} \times V^{*}, V^{*}=[0, \infty) \times \mathbb{R} \times B$, as follows: On each $[0, \infty) \times \rho^{-1}\left(B_{i}\right)$ with coordinates $(\tau, x, t)$ and contact form $d t+$ $e^{\tau} \beta_{0}(x)$, choose an $e^{\tau} \beta_{0}$-adapted almost complex structure $J_{0}$ on $[0, \infty) \times s_{i}\left(B_{i}\right)=$ $\{t=0\}$. This determines $J_{\kappa, n}$ which projects to $J_{0}$. By construction, we may also assume that the sections $s_{i}\left(B_{i}\right)$ and $s_{j}\left(B_{j}\right)$ differ by $t=$ const on the overlap $\rho^{-1}\left(B_{i} \cap B_{j}\right)$; hence the contact form on $[0, \infty) \times \rho^{-1}\left(B_{i} \cap B_{j}\right)$ is $d t+e^{\tau} \beta_{0}(x)$ with respect to either coordinate chart. This means that we can choose a $J_{0}$ on all of $[0, \infty) \times B$ and a $J_{\kappa, n}$ which projects to $J_{0}$ on all of $V^{*}$.

Step 4: Verification of conditions We now verify that $J_{\kappa, n}$ is tailored to $\left(M_{n}^{*}, \alpha_{n}^{*}\right)$. Conditions $\left(\mathrm{A}_{0}\right)$ and $\left(\mathrm{A}_{1}\right)$ are easily satisfied. It remains to verify $\left(\mathrm{A}_{2}\right)$, namely $J_{\kappa, n}$ is $d \beta_{ \pm}$-positive, where $\beta_{ \pm}$is the restriction of $\alpha_{n}$ to $R_{ \pm}\left(\Gamma_{n}\right)$. The reason this needs verification is that the adjustment in the vertical direction implies that the $t$-variable undergoes a coordinate change of the type $(t, y) \mapsto(t+f(y), y)$, where $y$ is a coordinate on $R_{ \pm}\left(\Gamma^{\prime}\right)$. By pulling back, we see that $\beta_{ \pm}(y)=d f(y)+\beta_{ \pm}^{\prime}(y)$, and $d \beta_{ \pm}=d \beta_{ \pm}^{\prime}$. Hence the $d \beta_{ \pm}$-positivity is inherited from the $d \beta_{ \pm}^{\prime}$-positivity.

Remark 4.9 Let $J_{0}$ be the projection of $J_{\kappa, n}$ onto $[0, \infty) \times B$. Since the 1 -forms $\beta_{0}(x)$ patch to give a contact 1 -form $\beta_{0}$ on $B$, it follows that $\left([0, \infty) \times B, d\left(e^{\tau} \beta_{0}\right)\right)$ is a (positive) symplectization and $J_{0}$ is adapted to the symplectization. Hence $\tau$ is a plurisubharmonic function with respect to $J_{0}$.

\subsection{Gluing along convex submanifolds}

In this section we discuss gluing along convex submanifolds. In particular, we carefully construct a contact 1 -form which is suited to counting holomorphic curves.

Let $\left(M^{\prime}, \Gamma^{\prime}, U\left(\Gamma^{\prime}\right), \xi^{\prime}\right)$ be a sutured contact manifold of dimension $2 m+1$ and $\alpha^{\prime}$ be an adapted contact 1 -form. Let $S_{1}$ and $S_{2}$ be two disjoint components of $\partial M^{\prime}$ and let $S_{i}^{ \pm}=S_{i} \cap R_{ \pm}\left(\Gamma^{\prime}\right)$. A neighborhood of $S_{i}^{+}$in $\left(M^{\prime}, \alpha^{\prime}\right)$ is given by $\left(S_{i}^{+} \times\right.$ $\left.[1-\varepsilon, 1], d t+\beta^{\prime}\right)$, where $t \in[1-\varepsilon, 1]$ and $S_{i}^{+}=S_{i}^{+} \times\{1\}$. Similarly, we have a neighborhood $\left(S_{i}^{-} \times[-1,-1+\varepsilon], d t+\beta^{\prime}\right)$ of $S_{i}^{-}=S_{i}^{-} \times\{-1\}$. Suppose there is a diffeomorphism $h: S_{1}^{+} \cup S_{1}^{-} \rightarrow S_{2}^{-} \cup S_{2}^{+}$which takes $\left(S_{1}^{+}, \beta^{\prime} \mid S_{1}^{+}\right)$to $\left(S_{2}^{-}, \beta^{\prime} \mid S_{2}^{-}\right)$ and $\left(S_{1}^{-}, \beta^{\prime} \mid S_{1}^{-}\right)$to $\left(S_{2}^{+}, \beta^{\prime} \mid S_{2}^{+}\right)$, and which can be extended to a (piecewise smooth) homeomorphism from $S_{1}$ to $S_{2}$. Also suppose that when we compose $\left.h\right|_{S_{1}^{+}}$and $h^{-1} \mid S_{2}^{+}$with the identifications of $\partial S_{i}^{-}$and $\partial S_{i}^{+}$by the flow of $\partial_{t}$ in $U\left(\Gamma^{\prime}\right)$, we get the identity on $\partial S_{1}^{+}$. 
Instead of gluing directly using $h$, we insert layers as follows: Fix $n>0$. Let $\left(M_{n}^{\prime}, \alpha_{n}^{\prime}\right)$ be the contact manifold obtained by gluing the products $\left(S_{1}^{+} \times[0, n], d t+\beta^{\prime} \mid S_{1}^{+}\right)$and $\left(S_{1}^{-} \times[0, n], d t+\beta^{\prime} \mid S_{1}^{-}\right)$to $\left(M^{\prime}, \alpha^{\prime}\right)$ by identifying $S_{1}^{+}$with $S_{1}^{+} \times\{0\}, S_{2}^{-}$with $S_{1}^{+} \times\{n\}, S_{2}^{+}$with $S_{1}^{-} \times\{0\}$, and $S_{1}^{-}$with $S_{1}^{-} \times\{n\}$.

We now construct the contact manifold $\left(M_{n}, \alpha_{n, f, g}\right)$ by filling in some of the boundary components of $\left(M_{n}^{\prime}, \alpha_{n}^{\prime}\right)$; see Figure 5. Here $f, g$ are smooth functions $[0,1] \sqcup \cdots \sqcup$ $[0,1] \rightarrow \mathbb{R}$, where there is a copy of $[0,1]$ for each component $V$ of $S_{1} \cap \Gamma$. Moreover, $f, g$ depend on $n$. Consider a boundary component of $\left(M_{n}^{\prime}, \alpha_{n}^{\prime}\right)$ of the form $V \times S^{1}$, where $V$ corresponds to a connected component of $S_{1} \cap \Gamma$ and $S^{1}=\mathbb{R} / 2 \pi \mathbb{Z}$ has coordinate $\theta$. The contact form $\alpha_{n}^{\prime}$ on $V \times S^{1}$ is given by $a_{n} d \theta+\beta_{0}^{\prime}$, where $a_{n}$ is a constant $>n / \pi$ and $\beta_{0}^{\prime}=\left.\alpha^{\prime}\right|_{V}$. We then fill $V \times S^{1}$ with $V \times D^{2}$, where we are using polar coordinates $(r, \theta)$ on $D^{2}$ and $S^{1}=\{r=1\}$. We require the contact form $\alpha_{n, f, g}$ on $V \times D^{2}$ to be of the form

$$
f(r) d \theta+g(r) \beta_{0}^{\prime},
$$

with boundary condition $(f(1), g(1))=(a, 1)$. The contact condition is equivalent to

$$
f^{\prime} g-g^{\prime} f=\left(f^{\prime}, g^{\prime}\right) \cdot(g,-f)>0,
$$

which in words says that the path $\{(f(r), g(r)), r \in[0,1]\}$, is transverse to radial rays in the $(f, g)$-plane and rotates clockwise around the origin. The Reeb vector field $R=R_{\alpha_{n, f, g}}$ is given by $R=\left(f^{\prime} R_{0}-g^{\prime} \partial_{\theta}\right) /\left(f^{\prime} g-g^{\prime} f\right)$, where $R_{0}$ is the Reeb vector field for $\beta_{0}^{\prime}$.

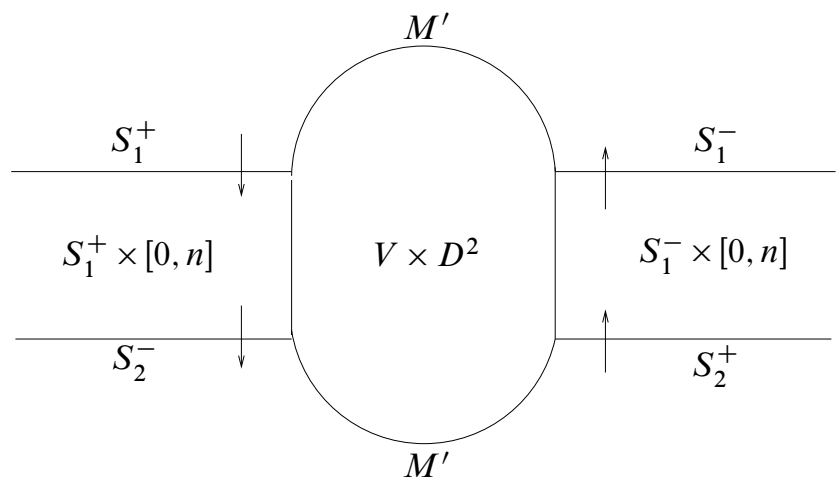

Figure 5: The convex gluing of $M^{\prime}$ near a component of $V$

We now choose specific $f$ and $g$ so that an orbit $\gamma$ of $R$ which passes through $M_{n}-M^{\prime}$ has action $A(\gamma)>n$. Let $B_{0}, B_{1}$ be large positive constants so that $B_{0}-a B_{1} \gg 0$. Then set 
- $(f(r), g(r))=\left(a r^{2}, B_{0}-a B_{1} r^{2}\right)$ for $r \in[0,1-2 \varepsilon]$;

- $0<f^{\prime}(r)$ and $g^{\prime}(r)<0$ for $r \in[1-2 \varepsilon, 1-\varepsilon]$;

- $f(r)=a, g^{\prime}(r)<0$ for $r \in[1-\varepsilon, 1]$;

- $(f(1), g(1))=(a, 1)$;

- $f(r)=a, g(r)=e^{1-r}$ for $r \in[1-\varepsilon / 2,1]$.

The last condition is to ensure the smooth gluing of $f d \theta+g \beta_{0}^{\prime}$ with $\alpha_{n}^{\prime}$.

If $\gamma$ passes through $M_{n}^{\prime}-M^{\prime}$, then we claim that $A(\gamma)>n$ by construction. Suppose $\gamma$ lies in $V \times S^{1}$. Then we compute $R=\left(1 / B_{0}\right) R_{0}+\left(B_{1} / B_{0}\right) \partial_{\theta}$ for $0 \leq r \leq 1-2 \varepsilon$. Since $B_{0} / B_{1} \gg a$, it takes at least $2 \pi a$ units of time to travel once around the $\theta-$ direction; hence $A(\gamma)>n$. (When $r=0$, then $R=\left(1 / B_{0}\right) R_{0}$ and $\gamma$ is tangent to $V \times\{0\}$. If $B_{0}$ is sufficiently large, then $A(\gamma)>n$.) On the other hand, for $r \in[1-2 \varepsilon, 1]$, the coefficient in front of $\partial_{\theta}$ in $R$ is less than $1 / f \approx 1 / a$; hence $A(\gamma)>n$ for sufficiently small $\varepsilon$.

Finally, note that the above construction depends continuously on $(n, f, g)$. Hence, by Moser's Lemma, $\left(M_{n}, \operatorname{ker} \alpha_{n, f, g}\right) \simeq\left(M_{n^{\prime}}, \operatorname{ker} \alpha_{n^{\prime}, f^{\prime}, g^{\prime}}\right)$ for any two triples $(n, f, g)$, $\left(n^{\prime}, f^{\prime}, g^{\prime}\right)$. In particular $\left(M_{n}, \operatorname{ker} \alpha_{n, f, g}\right) \simeq(M, \xi)$, where $(M, \xi)$ is obtained from $\left(M^{\prime}, \xi^{\prime}\right)$ by gluing $S_{1}$ to $S_{2}$.

Summarizing the above discussion, we have:

Lemma 4.10 Let $(M, \xi)$ be a compact contact manifold of dimension $2 m+1$ and $\left(S, \Gamma_{S}\right) \subset(M, \xi)$ be a convex submanifold. If $\left(M^{\prime}, \xi^{\prime}\right)$ is obtained from $(M, \xi)$ by cutting along $S$, then, for any $n>0$ and appropriate $f=f(n), g=g(n),(M, \xi)$ is contactomorphic to $\left(M_{n}, \operatorname{ker} \alpha_{n, f, g}\right)$, where $\alpha_{n, f, g}$ is obtained from a contact 1form $\alpha^{\prime}$ adapted to $\left(M^{\prime}, \Gamma^{\prime}, U\left(\Gamma^{\prime}\right), \xi^{\prime}\right)$ by attaching (i) layers $\left(S_{1}^{+} \times[0, n], d t+\beta^{\prime} \mid S_{1}^{+}\right)$ and $\left(S_{1}^{-} \times[0, n], d t+\beta^{\prime} \mid S_{1}^{-}\right)$and (ii) $\left(V \times S^{1}, f d \theta+g \beta_{0}^{\prime}\right)$. Moreover, the Reeb vector field $R=R_{\alpha_{n, f, g}}$ satisfies the following:

- Every orbit of $R$ which intersects $M_{n}-M^{\prime}$ has action larger than $n$.

- $R$ is tangent to $V \times\{0\}$, positively transverse to $S_{i}^{+} \times\{t\}$ and $S_{i}^{-} \times\{t\}$ for all $t \in[0, n]$, and transverse to $\theta=$ const on $V \times\left(D^{2}-\{0\}\right)$.

When $\operatorname{dim} M=3$, the dividing set $V \times\{0\}$ is a periodic orbit of $R$.

We define the tailored almost complex structure $J=J_{n, f, g}$ on $\mathbb{R} \times M_{n}^{*}$ as follows: Choose a tailored $J^{\prime}$ on $\mathbb{R} \times\left(M^{\prime}\right)^{*}$ so that its restrictions to $S_{1}^{+}$and $S_{2}^{-}$, and also its restrictions to $S_{1}^{-}$and $S_{2}^{+}$, agree via $h$. We then extend $J^{\prime}$ to $J$ on $\mathbb{R} \times S_{1}^{ \pm} \times[0, n]$ so that $J$ is invariant in both the $s$-and $t$-directions. Finally, we extend $J$ so that it is $\alpha_{n, f, g}$-adapted on $\mathbb{R} \times V \times D^{2}$. 


\section{Compactness results}

Let $(M, \alpha)$ be a sutured contact manifold, and let $\left(M^{*}, \alpha^{*}\right)$ denote its completion as defined in Section 2.4. Let $J$ be an almost complex structure on $\mathbb{R} \times M^{*}$ tailored to $\left(M^{*}, \alpha^{*}\right)$, as defined in Section 3.1. In this section we show that the SFT compactness theorem for holomorphic curves in the symplectization of a closed contact manifold, proved by Bourgeois et al [3] and Cieliebak and Mohnke [6], extends to the case of $J-$ holomorphic curves in $\mathbb{R} \times M^{*}$. At the end of this section, we extend the compactness theorem for embedded contact homology of the last author [24] to $\mathbb{R} \times M^{*}$ in the case $\operatorname{dim}(M)=3$.

\subsection{Convergence of stable Riemann surfaces}

We begin by reviewing some notation and classical results about the convergence of stable Riemann surfaces, following [3].

A marked Riemann surface is a triple $\mathbf{S}=(\Sigma, j, \mathbf{m})$ consisting of a closed Riemann surface $(\Sigma, j)$ and a finite ordered set $\mathbf{m} \subset \Sigma$ of "punctures" or "marked points". (The surface $\Sigma$ does not need to be connected.) Two marked Riemann surfaces $\mathbf{S}=(\Sigma, j, \mathbf{m})$ and $\mathbf{S}^{\prime}=\left(\Sigma^{\prime}, j^{\prime}, \mathbf{m}^{\prime}\right)$ are said to be equivalent if there exists a diffeomorphism $\varphi: \Sigma \stackrel{\sim}{\rightarrow} \Sigma^{\prime}$ such that $\varphi_{*} j=j^{\prime}$ and $\varphi(\mathbf{m})=\mathbf{m}^{\prime}$ in an order-preserving way. The surface $\mathbf{S}$ is called stable if, on each connected component $\Sigma_{0}$ of $\Sigma$, we have $2 g\left(\Sigma_{0}\right)+\mu\left(\Sigma_{0}\right) \geq 3$. Here $g\left(\Sigma_{0}\right)$ is the genus of $\Sigma_{0}$ and $\mu\left(\Sigma_{0}\right)$ is the number of marked points on $\Sigma_{0}$. A nodal Riemann surface is a quadruple $\mathbf{S}=(\Sigma, j, \mathbf{m}, D)$, where $(\Sigma, j, \mathbf{m})$ is a marked Riemann surface as before, and $D \subset \Sigma \backslash \mathbf{m}$ is a finite set partitioned into unordered pairs $\left\{\left(d_{i}^{\prime}, d_{i}^{\prime \prime}\right)\right\}$. A stable nodal Riemann surface is defined as above, where the set of marked points is taken to be $\mathbf{m} \sqcup D$. From a nodal surface $\mathbf{S}=(\Sigma, j, \mathbf{m}, D)$ one can form a singular surface $\widehat{\Sigma}_{D}=\Sigma /\left(d_{i}^{\prime} \sim d_{i}^{\prime \prime}\right)$.

Let $\mathbf{S}=(\Sigma, j, \mathbf{m})$ be a stable marked Riemann surface. On $\dot{\Sigma}=\Sigma \backslash \mathbf{m}$ there is a unique complete, finite volume hyperbolic metric $h^{j, \mathbf{m}}$ which is compatible with $j$. Denote its injectivity radius by $\rho$. Given $\epsilon>0$, we define the "thick part" and "thin part"

$$
\begin{aligned}
\operatorname{Thick}_{\epsilon}(\mathbf{S}) & =\{x \in \dot{\Sigma} \mid \rho(x) \geq \epsilon\}, \\
\operatorname{Thin}_{\epsilon}(\mathbf{S}) & =\overline{\{x \in \dot{\Sigma} \mid \rho(x)<\epsilon\} .}
\end{aligned}
$$

If $\epsilon<\log (1+\sqrt{2})$, then each component of $\operatorname{Thin}_{\epsilon}(\mathbf{S})$ is conformally equivalent to a punctured disk or to a finite cylinder. Each cylindrical component $C$ of $\operatorname{Thin}_{\epsilon}(\mathbf{S})$ contains a unique closed geodesic $\Gamma_{C}$. The thick and thin parts of complete, finite volume hyperbolic metrics for stable nodal Riemann surfaces are defined similarly, except that we take $\dot{\Sigma}=\Sigma \backslash(\mathbf{m} \cup D)$. 
Definition 5.1 A sequence of marked Riemann surfaces $\mathbf{S}_{n}=\left(\Sigma_{n}, j_{n}, \mathbf{m}_{n}\right)$ converges to a nodal Riemann surface $\mathbf{S}=(\Sigma, j, \mathbf{m}, D)$ if the following hold:

- There exist a smooth surface $\Sigma^{D}$, diffeomorphisms $\varphi_{n}: \Sigma^{D} \stackrel{\sim}{\rightarrow} \Sigma_{n}$ and an ordered set $\mathbf{m}^{D} \subset \Sigma^{D}$ such that $\varphi_{n}\left(\mathbf{m}^{D}\right)=\mathbf{m}_{n}$ (as ordered sets).

- There exist disjoint circles $\Gamma_{1}, \ldots, \Gamma_{k} \subset \Sigma^{D} \backslash \mathbf{m}^{D}$ and a map $\varphi: \Sigma^{D} \rightarrow \widehat{\Sigma}_{D}$ such that $\varphi$ is a diffeomorphism between $\Sigma^{D} \backslash \bigcup \Gamma_{i}$ and $\Sigma \backslash D$, and $\varphi\left(\mathbf{m}^{D}\right)=\mathbf{m}$ (as ordered sets).

- $\varphi_{n}\left(\Gamma_{i}\right) \subset \Sigma_{n}$ are closed geodesics for the metric $h^{j_{n}, \mathbf{m}_{n}}$ and are contained in the thin part (defined using some $\epsilon<\log (1+\sqrt{2})$ ).

- $\varphi_{n}^{*} j_{n} \rightarrow \varphi^{*} j$ in $C_{\mathrm{loc}}^{\infty}\left(\Sigma^{D} \backslash \bigcup \Gamma_{i}\right)$ or, equivalently, $\varphi_{n}^{*}\left(h^{j_{n}, \mathbf{m}_{n}}\right) \rightarrow \varphi^{*}\left(h^{j, \mathbf{m}}\right)$ in $C_{\mathrm{loc}}^{\infty}\left(\Sigma^{D} \backslash\left(\bigcup \Gamma_{i} \cup \mathbf{m}^{D}\right)\right)$.

- Given a point $c_{i} \in \Gamma_{i}$, the geodesic $\operatorname{arcs} \delta_{i}^{n}$ for the metric $\varphi_{n}^{*}\left(h^{j_{n}, \mathbf{m}_{n}}\right)$ which intersect $\Gamma_{i}$ orthogonally at $c_{i}$ and whose endpoints are contained in the thick part of $\Sigma^{D}$ for the metric $\varphi_{n}^{*}\left(h^{j_{n}, \mathbf{m}_{n}}\right)$, converge uniformly as $n \rightarrow \infty$ to a continuous arc in $\Sigma^{D}$ which passes through $c_{i}$ and is a geodesic in $\Sigma^{D} \backslash\left(\bigcup \Gamma_{i} \cup \mathbf{m}^{D}\right)$ for the metric $\varphi^{*}\left(h^{j, \mathbf{m}}\right)$.

We then have the following theorem (see [3, Theorem 4.2] and the references therein):

Theorem 5.2 Any sequence of stable marked Riemann surfaces $\mathbf{S}_{n}=\left(\Sigma_{n}, j_{n}, \mathbf{m}_{n}\right)$ with fixed $2 g\left(\Sigma_{n}\right)+\mu\left(\Sigma_{n}\right)$ has a subsequence which converges to a nodal Riemann surface $\mathbf{S}=(\Sigma, j, \mathbf{m}, D)$.

Fact 5.3 Let $g_{n}$ be a sequence of Riemannian metrics which converges uniformly to a Riemannian metric $g$. Let $l_{n}$ be the length functional for $g_{n}$ and $l$ be the length functional for $g$. Then for any $\delta>0$ there exists $n_{0} \in \mathbb{N}$ such that for all $n \geq n_{0}$ and for all arcs $\gamma$ we have

$$
(1-\delta) l(\gamma) \leq l_{n}(\gamma) \leq(1+\delta) l(\gamma)
$$

The proof of Fact 5.3 is an easy exercise.

Proposition 5.4 Let $\mathbf{S}_{n}=\left(\Sigma_{n}, j_{n}, \mathbf{m}_{n}\right)$ be a sequence of Riemann surfaces which converges to a nodal Riemann surface $\mathbf{S}=(\Sigma, j, \mathbf{m}, D)$, in the sense of Definition 5.1. Then for all $\epsilon, \delta>0$ there is $n_{0} \in \mathbb{N}$ such that

$$
\begin{gathered}
\varphi^{-1}\left(\operatorname{Thick}_{\epsilon}(\mathbf{S})\right) \subset \varphi_{n}^{-1}\left(\operatorname{Thick}_{(1-\delta) \epsilon}\left(\mathbf{S}_{n}\right)\right), \\
\varphi^{-1}\left(\operatorname{Thin}_{\epsilon}(\mathbf{S})\right) \subset \varphi_{n}^{-1}\left(\operatorname{Thin}_{(1+\delta) \epsilon}\left(\mathbf{S}_{n}\right)\right)
\end{gathered}
$$

for all $n \geq n_{0}$. 
Proof Let $h_{n}$ be the complete, finite volume hyperbolic metric on $\Sigma^{D} \backslash \mathbf{m}^{D}$ which is compatible with $\varphi_{n}^{*} j_{n}$, and $h$ be the complete, finite volume hyperbolic metric on $\Sigma^{D} \backslash\left(\mathbf{m}^{D} \cup \Gamma_{i}\right)$ which is compatible with $\varphi^{*} j$. Also let $\rho_{n}$ and $\rho$ be the injectivity radii of $h_{n}$ and $h$, respectively. Let $z \in \Sigma^{D} \backslash\left(\mathbf{m}^{D} \cup \Gamma_{i}\right)$. In order to compute the injectivity radii at $z$, it suffices to compute the shortest geodesic loops based at $z$ (see for example Hummel [23, Lemma 4.8]). Let $\gamma$ be the shortest $g$-geodesic loop based at $z$, and let $\gamma_{n}$ be the shortest $g_{n}$-geodesic loop based at $z$. By Fact 5.3, we have

$$
(1-\delta) l(\gamma) \leq(1-\delta) l\left(\gamma_{n}\right) \leq l_{n}\left(\gamma_{n}\right),
$$

for sufficiently large $n$. Hence $(1-\delta) \rho(z) \leq \rho_{n}(z)$. We then conclude that $\rho_{n}(z) \geq$ $(1-\delta) \epsilon$ whenever $\rho(z) \geq \epsilon$.

On the other hand, if $l(\gamma)<\epsilon$, then we have

$$
l_{n}\left(\gamma_{n}\right) \leq l_{n}(\gamma) \leq(1+\delta) l(\gamma),
$$

for sufficiently large $n$. We then conclude that $\rho_{n}(z) \leq(1+\delta) \epsilon$ whenever $\rho(z)<\epsilon$.

\subsection{Holomorphic curves in $\mathbb{R} \times M^{*}$}

Let $J$ be a tailored almost complex structure on $\mathbb{R} \times M^{*}$ as usual. Let $(\Sigma, j, \mathbf{m})$ be a marked Riemann surface. The notation

$$
F=(a, f):(\Sigma, j, \mathbf{m}) \rightarrow\left(\mathbb{R} \times M^{*}, J\right)
$$

denotes a $(j, J)$-holomorphic map from the punctured Riemann surface $\dot{\Sigma}=\Sigma \backslash \mathbf{m}$ to $M^{*}$. If $p \in \mathbf{m}$, and if $\gamma$ is a Reeb orbit of $\alpha$, we say that $F$ is "positively asymptotic" to $\gamma$ at $p$ if $\lim _{z \rightarrow p} a(z)=+\infty$ and if the restriction of $f$ to a circle around $p$ converges to $\gamma$ as the size of the circle converges to zero. We also say that $p$ is a "positive puncture" of $F$ asymptotic to $\gamma$. We define "negatively asymptotic" analogously but with $\lim _{z \rightarrow p} a(z)=-\infty$.

Now let $\underline{\gamma}=\left(\gamma_{1}, \ldots, \gamma_{k}\right)$ and $\underline{\gamma}^{\prime}=\left(\gamma_{1}^{\prime}, \ldots, \gamma_{l}^{\prime}\right)$ be finite ordered lists of Reeb orbits, possibly with repetitions. Let $\overline{\mathcal{M}}_{g}\left(\underline{\gamma} ; \underline{\gamma}^{\prime} ; J\right)$ denote the moduli space of holomorphic maps $F$ as above such that $\Sigma$ has genus $g$, there are $k+l$ marked points in $\mathbf{m}, F$ is positively asymptotic to $\gamma_{i}$ at the $i$-th marked point, and $F$ is negatively asymptotic to $\gamma_{j}^{\prime}$ at the $(k+j)$-th marked point.

We wish to extend the SFT compactness theorem to sequences of holomorphic curves in these moduli spaces. To do so, it is sufficient to show that for any sequence of such curves, the projections to $M^{*}$ are confined in a compact set.

We first show that a sequence of holomorphic curves cannot escape from the side (S). Recall the functions $\tau: M^{*} \rightarrow[-1, \infty)$ and $t: M^{*} \rightarrow(-\infty, \infty)$ from Section 2.4. 
We extend $\tau$ and $t$ to maps $\mathbb{R} \times M^{*} \rightarrow[-1, \infty)$ and $\mathbb{R} \times M^{*} \rightarrow(-\infty, \infty)$ by precomposing with the projection $\mathbb{R} \times M^{*} \rightarrow M^{*}$ onto the second factor.

Lemma 5.5 Let $F \in \mathcal{M}_{g}\left(\underline{\gamma} ; \underline{\gamma}^{\prime} ; J\right)$ for some $g$, $\underline{\gamma}$, and $\underline{\gamma^{\prime}}$. Then $\tau \circ F(z) \leq 0$ for all $z \in \dot{\Sigma}$.

Proof Suppose there exists a point $z \in \dot{\Sigma}$ such that $\tau \circ F(z)>0$. Since $\tau<0$ along the closed Reeb orbits, it follows that $\tau \circ F$ has a positive local maximum, which we assume without loss of generality to be attained at $z$.

The projection of $J$ to $\widehat{R_{+}(\Gamma)}$ is $J_{0}$. Hence where $\tau>0$, the projection of $F$ to $\widehat{R_{+}(\Gamma)}$ is a $J_{0}$-holomorphic map. Since $\tau$ restricted to $\widehat{R_{+}(\Gamma)}$ is a plurisubharmonic function where it is positive, a positive local maximum of $\tau \circ F$ is forbidden by the maximum principle.

The main task in the rest of this section is to show that a sequence of holomorphic curves cannot escape from the top (T) or bottom (B). That is, we wish to bound the restriction to a holomorphic curve of the absolute value of the function $t: M^{*} \rightarrow \mathbb{R}$ from Section 2.4. For this purpose we need to consider somewhat more general holomorphic curves than the ones in $\mathcal{M}_{g}\left(\bar{\gamma} ; \bar{\gamma}^{\prime} ; J\right)$, in particular the restrictions of such curves to certain subsets of the domain. However our curves $F$ will always have an upper bound on $\tau \circ F$ as a result of Lemma 5.5. In addition, all our curves $F$ will have finite Hofer energy $E(F)$; see [3, Section 5.3] for the definition of Hofer energy and [3, Proposition 5.13] for the proof that any curve in $\mathcal{M}_{g}\left(\bar{\gamma} ; \bar{\gamma}^{\prime} ; J\right)$ (and consequently the restriction of any such curve to a subset of the domain) has finite Hofer energy.

\subsection{The Stein case}

It is easiest to obtain a bound on $|t|$ when $\left(\widehat{R_{ \pm}(\Gamma)}, J_{0}, \widehat{\beta}_{ \pm}\right)$is a Stein manifold. (Recall that we can arrange to be in this situation when $\widehat{R_{ \pm}(\Gamma)}$ is a surface.) In this case we have $|t| \leq 1$ by the following lemma and corollary.

Lemma 5.6 Suppose $J_{0}$ is an integrable complex structure making $\left(\widehat{R_{ \pm}(\Gamma)}, J_{0}, \widehat{\beta}_{ \pm}\right)$ into a Stein manifold. If $F:(\Sigma, j, \mathbf{m}) \rightarrow\left(\mathbb{R} \times M^{*}, J\right)$ is a holomorphic map, then $t \circ F$ is a harmonic function on the open set $\{z \in \dot{\Sigma}:|t \circ F(z)|>1\}$.

Proof We prove the lemma for the case when $(t \circ F)(z)>1$; the argument for the case $(t \circ F)(z)<-1$ is identical. The symplectization of the top (T) is written as $\mathbb{R} \times(1, \infty) \times \widehat{R_{+}(\Gamma)}$, with coordinates $s$ on $\mathbb{R}$ and $t$ on $(1, \infty)$. On $(1, \infty) \times \widehat{R_{+}(\Gamma)}$, we may take the contact form to be $\alpha=d t+\beta$, where $\beta=\widehat{\beta}_{+}$. Since $\left(\widehat{R_{+}(\Gamma)}, J_{0}, \beta\right)$ 
is Stein, $\beta \circ J_{0}=d f$, where $f$ is the strictly plurisubharmonic function. With these conventions in place, we compute the Laplacian of $t \circ F$ :

$$
\begin{aligned}
d d^{\mathbb{C}}(t \circ F) & =d(d(t \circ F) \circ j)=d\left(F^{*}(d t \circ J)\right)=F^{*} d((\alpha-\beta) \circ J) \\
& =F^{*} d(d s-(\beta \circ J))=-F^{*} d(\beta \circ J) .
\end{aligned}
$$

We now claim that $\beta \circ J=d f$. First we observe that $\beta \circ J$ and $d f$ both evaluate to zero on $\partial_{s}$ and $\partial_{t}$. Next we compare $(\beta \circ J)(X)$ and $\left(\beta \circ J_{0}\right)(X)=d f(X)$ for any vector $X$ tangent to $\widehat{R_{+}(\Gamma)}$. Since $J(X)=J_{0}(X)+v_{0}(X) \partial_{s}+v_{1}(X) \partial_{t}$ by the definition of $J$ and $\beta\left(\partial_{s}\right)=\beta\left(\partial_{t}\right)=0$, it follows that $(\beta \circ J)(X)=\left(\beta \circ J_{0}\right)(X)$.

Finally, since $\beta \circ J$ is exact, we conclude that $d d^{\mathbb{C}}(t \circ F)=0$.

Corollary 5.7 Suppose $J_{0}$ is an integrable complex structure making $\left(\widehat{R_{ \pm}(\Gamma)}, J_{0}, \widehat{\beta}_{ \pm}\right)$ into a Stein manifold. If $F \in \mathcal{M}_{g}\left(\underline{\gamma} ; \underline{\gamma^{\prime}} ; J\right)$, then $|(t \circ F)(z)| \leq 1$ for all $z \in \dot{\Sigma}$.

Proof If there is a point $z \in \dot{\Sigma}$ such that $|(t \circ F)(z)|>1$, then there is a local maximum for $t \circ F$, which we may assume to be attained at $z$. But this is forbidden by the maximum principle because $t \circ F$ is harmonic in a neighborhood of $z$ by Lemma 5.6.

The non-Stein case is less nice because we do not necessarily have $|t| \leq 1$. However we can still obtain a theoretical upper bound on $|t|$, as the rest of this section will explain.

\subsection{Bubbling lemma}

In this section we adapt the usual bubbling argument to our noncompact setting; cf [3, Lemma 5.11].

Let $\mathbf{S}=(\Sigma, j, \mathbf{m})$ be a marked Riemann surface, and let

$$
F=(a, f):(\Sigma, j, \mathbf{m}) \rightarrow\left(\mathbb{R} \times M^{*}, J\right)
$$

be a holomorphic map as above. Below, we write $b=t \circ f: \dot{\Sigma} \rightarrow \mathbb{R}$. When the image of $F$ is contained in the symplectization $\mathbb{R} \times(1,+\infty) \times \widehat{R_{+}(\Gamma)}$ of the Top (or in the symplectization $\mathbb{R} \times(-\infty,-1) \times \widehat{R_{-}(\Gamma)}$ of the Bottom), we can write $f=(b, v)$, where $v: \dot{\Sigma} \rightarrow \widehat{R_{ \pm}(\Gamma)}$.

On $\mathbb{R} \times M^{*}$, we will always use the Riemannian metric

$$
g=d s \otimes d s+\alpha^{*} \otimes \alpha^{*}+d \alpha^{*}(\cdot, J \cdot)-d \alpha^{*}(J \cdot, \cdot),
$$


where $s$ is the $\mathbb{R}$-coordinate. (The last term is added to symmetrize the tensor, since we are only taking $J$ to be $d \alpha$-positive.) With respect to this metric, $\|\nabla t\|$ is uniformly bounded.

Also recall the following (by now well-known) topological lemma.

Lemma 5.8 (Hofer's lemma) Let $(X, d)$ be a complete metric space, $f: X \rightarrow \mathbb{R}$ be a nonnegative continuous function, $x_{0} \in X$, and $\delta>0$. Then there exist $x \in X$ and a positive number $\varepsilon \leq \delta$ such that

$$
d\left(x_{0}, x\right)<2 \delta, \quad \sup _{B_{\varepsilon}(x)} f \leq 2 f(x), \quad \varepsilon f(x) \geq \delta f\left(x_{0}\right),
$$

where $B_{\varepsilon}(x)$ is an open ball of radius $\varepsilon$ about $x$.

Let us write $\mathbb{D}_{r}=\{z \in \mathbb{C}|| z \mid<r\}$, and $\mathbb{D}=\mathbb{D}_{1}$. Then we have the following:

Lemma 5.9 (Bubbling) Consider a sequence of $J$-holomorphic maps

$$
F_{n}=\left(a_{n}, f_{n}\right): \mathbb{D} \rightarrow\left(\mathbb{R} \times M^{*}, J\right)
$$

satisfying $E\left(F_{n}\right)<C$ and $\tau \circ F_{n}<C^{\prime}$ for some constants $C^{\prime}, C>0$. Suppose that $\left\|\nabla F_{n}(0)\right\| \rightarrow \infty$ as $n \rightarrow \infty$. Then after passing to a subsequence, there exists a sequence of points $x_{n} \in \mathbb{D}$ converging to 0 , and sequences of positive numbers $c_{n}, R_{n} \rightarrow \infty$ such that $\left|x_{n}\right|+c_{n}^{-1} R_{n}<1$ and the rescaled maps

$$
\begin{aligned}
F_{n}^{0}: \mathbb{D}_{R_{n}} & \longrightarrow\left(\mathbb{R} \times M^{*}, J\right), \\
z & \longmapsto F_{n}\left(x_{n}+c_{n}^{-1} z\right)
\end{aligned}
$$

converge in $C_{\mathrm{loc}}^{\infty}(\mathbb{C})$ to one of the following:

(1) a nonconstant holomorphic map $F^{0}: \mathbb{C} \rightarrow\left(\mathbb{R} \times M^{*}, J\right)$, after translating in the $s$-direction, or

(2) a nonconstant holomorphic map $F^{0}: \mathbb{C} \rightarrow\left(\mathbb{R} \times \mathbb{R} \times \widehat{R_{ \pm}(\Gamma)}, J\right)$, after translating in the $s$ - and $t$-directions.

In both cases the limit map satisfies the condition $E\left(F^{0}\right) \leq C$.

The gradients $\left\|\nabla F_{n}(0)\right\|$ are computed with respect to the standard Euclidean metric on $\mathbb{D}$ and the metric on $\mathbb{R} \times M^{*}$ given by Equation (15). 
Proof Choose a sequence $\delta_{n}>0$ such that $\delta_{n} \rightarrow 0$ and $\delta_{n}\left\|\nabla F_{n}(0)\right\| \rightarrow \infty$. Applying Hofer's lemma to $\left\|\nabla F_{n}\right\|$, we obtain new sequences $x_{n} \in \mathbb{D}$ and $0<\varepsilon_{n} \leq \delta_{n}$ such that $x_{n} \rightarrow 0$ and

$$
\sup _{\left|x-x_{n}\right| \leq \varepsilon_{n}}\left\|\nabla F_{n}(x)\right\| \leq 2\left\|\nabla F_{n}\left(x_{n}\right)\right\|, \quad \varepsilon_{n}\left\|\nabla F_{n}\left(x_{n}\right)\right\| \rightarrow \infty .
$$

Set $c_{n}=\left\|\nabla F_{n}\left(x_{n}\right)\right\|$ and $R_{n}=\varepsilon_{n}\left\|\nabla F_{n}\left(x_{n}\right)\right\|$. For sufficiently large $n$ we have $\left|x_{n}\right|+c_{n}^{-1} R_{n}<1$. Hence there exist rescaled maps

$$
F_{n}^{0}(z)=\left(a_{n}^{0}(z), f_{n}^{0}(z)\right)=\left(a_{n}\left(x_{n}+c_{n}^{-1} z\right)-a_{n}\left(x_{n}\right), f_{n}\left(x_{n}+c_{n}^{-1} z\right)\right),
$$

defined on $\mathbb{D}_{R_{n}}$. The sequence $\left\{F_{n}^{0}\right\}$ satisfies

- $a_{n}^{0}(0)=0$;

- $\left\|\nabla F_{n}^{0}(0)\right\|=1$;

- $\sup _{z \in \mathbb{D}_{R_{n}}}\left\|\nabla F_{n}^{0}(z)\right\| \leq 2$;

- $E\left(F_{n}^{0}\right) \leq E\left(F_{n}\right) \leq C$.

We now consider two cases:

Case 1 If there is a constant $C>0$ such that $\left|b_{n}\left(x_{n}\right)\right| \leq C$, then the maps $F_{n}^{0}$ are uniformly bounded, in the sense that for any compact set $K_{1} \subset \mathbb{C}$ there is a compact set $K_{2} \subset \mathbb{R} \times M^{*}$ such that $F_{n}^{0}\left(K_{1}\right) \subset K_{2}$ for all $n$ sufficiently large. This is a consequence of the uniform bounds on $\left\|\nabla F_{n}^{0}\right\|$ and on $\tau \circ F_{n}^{0}$. The Gromov-Schwarz lemma [3, Lemma 5.1] implies that all the derivatives of $F_{n}^{0}$ are bounded. Hence we can apply the Arzelà-Ascoli theorem and extract a subsequence which converges in $C_{\text {loc }}^{\infty}(\mathbb{C})$ to a finite energy plane

$$
F^{0}: \mathbb{C} \rightarrow\left(\mathbb{R} \times M^{*}, J\right) .
$$

(In the rest of the paper, the Gromov-Schwarz lemma and the Arzelà-Ascoli theorem will be used repeatedly without specific mention.) The limiting map $F^{0}$ is nonconstant since $\left\|\nabla F_{n}^{0}(0)\right\|=1$ for all $n$.

Case 2 Suppose that $b_{n}\left(x_{n}\right)$ is unbounded. Then, without loss of generality, we can assume that $\lim _{n \rightarrow+\infty} b\left(x_{n}\right)=+\infty$ and that there exists a sequence $R_{n}^{\prime} \leq R_{n}$ such that

$$
\lim _{n \rightarrow \infty} R_{n}^{\prime}=+\infty \quad \text { and } \quad F_{n}^{0}\left(\mathbb{D}_{R_{n}^{\prime}}\right) \subset \mathbb{R} \times(1, \infty) \times \widehat{R_{+}(\Gamma)} \subset \mathbb{R} \times M^{*} .
$$

Therefore $F_{n}^{0}$ can be viewed as a map

$$
F_{n}^{0}=\left(a_{n}^{0}, b_{n}^{0}, v_{n}^{0}\right): \mathbb{D}_{R_{n}^{\prime}} \rightarrow \mathbb{R} \times \mathbb{R} \times \widehat{R_{+}(\Gamma)} .
$$


If we define

$$
\widetilde{F}_{n}^{0}(z)=\left(a_{n}^{0}(z)-a_{n}^{0}(0), b_{n}^{0}(z)-b_{n}^{0}(0), v_{n}^{0}(z)\right),
$$

then the uniform bound on the gradient implies that for any compact set $K \subset \mathbb{C}$ there is a positive constant $C$ such that $\widetilde{F}_{n}^{0}(K) \subset[-C, C] \times[-C, C] \times \widehat{R_{+}(\Gamma)}$. Hence there is a subsequence which converges in $C_{\mathrm{loc}}^{\infty}(\mathbb{C})$ to a nonconstant finite energy plane

$$
\widetilde{F}^{0}: \mathbb{C} \rightarrow\left(\mathbb{R} \times \mathbb{R} \times \widehat{R_{+}(\Gamma)}, J\right) .
$$

This completes the proof of Lemma 5.9.

\subsection{Bound on the $t$-coordinate}

In this section we discuss the bound on the $t$-coordinate and the removal of singularities.

5.5.1 Gradient bound for a single curve We start this section with the following useful lemma.

Lemma 5.10 Let $\left(M^{*}, \alpha^{*}\right)$ be the completion of a sutured contact manifold $(M, \alpha)$, and let $J$ be a tailored almost complex structure on $\mathbb{R} \times M^{*}$.

(1) If $F=(a, f): \mathbb{C} \rightarrow\left(\mathbb{R} \times M^{*}, J\right)$ is a finite energy holomorphic map with bounded gradient and $\int_{\mathbb{C}} f^{*} d \alpha^{*}=0$, then $F$ is constant.

(2) If $F=(a, f)$ : $\mathbb{C}^{\times}=\mathbb{C}-\{0\} \rightarrow\left(\mathbb{R} \times M^{*}, J\right)$ is a finite energy holomorphic map with bounded gradient, $R_{\alpha^{*}}$ has no closed orbits, and $\int_{\mathbb{C}^{\times}} f^{*} d \alpha^{*}=0$, then $F$ is constant.

In (2) the gradient is computed using the flat metric on $\mathbb{C}^{\times}$, viewed as an infinite cylinder.

Proof (1) The first statement is basically [19, Lemma 28], which goes through without modification to our noncompact case. By the zero $d \alpha^{*}$-energy condition, $\operatorname{Im}(F)$ is contained in $\mathbb{R} \times \gamma$, where $\gamma$ is a Reeb orbit of $R_{\alpha^{*}}$. Let $\tilde{\gamma}$ be the universal cover of $\gamma$ if $\gamma \simeq S^{1}$ or $\mathbb{R}$, and let $\tilde{\gamma} \simeq \mathbb{R}$ be the extension of $\gamma$ to $M^{*}$ if $\gamma$ is an interval. Then $F$ factors through a holomorphic map $\phi: \mathbb{C} \rightarrow \mathbb{C}=\mathbb{R} \times \tilde{\gamma}$. Note that $\nabla F$ is bounded if and only if $\partial \phi / \partial z$ is bounded with respect to the flat metric on both $\mathbb{C}$ 's. It then follows that $\partial \phi / \partial z$ is bounded and hence constant. Therefore $\phi(z)=c_{0}+c_{1} z$ for some constants $c_{0}, c_{1}$, and the corresponding $F$ does not have finite Hofer energy unless $c_{1}=0$.

(2) If $R_{\alpha^{*}}$ has no closed orbits, then the map $F$ factors through a holomorphic map $\phi: \mathbb{C}^{\times} \rightarrow \mathbb{C}=\mathbb{R} \times \tilde{\gamma}$, where $\tilde{\gamma} \simeq \mathbb{R}$. First observe that any holomorphic function $\phi(z)$ on $\mathbb{C}^{\times}$can be written as a Laurent series $\sum_{n \in \mathbb{Z}} a_{n} z^{n}, a_{n} \in \mathbb{C}$, where 
$\phi_{0}(z)=\sum_{n \geq 1} a_{n} z^{n}$ is a holomorphic function on $\mathbb{C}$ and $\phi_{\infty}(z)=\sum_{n \leq-1} a_{n} z^{n}$ is a holomorphic function on $\mathbb{C}^{\times} \cup\{\infty\}$, and both $\phi_{0}$ and $\phi_{\infty}$ have infinite radius of convergence. Next observe that the boundedness of $\nabla F$ is equivalent to the boundedness of

$$
\frac{\partial}{\partial w}(\phi \circ g(w))=\frac{\partial \phi}{\partial z}\left(e^{w}\right) \cdot e^{w}=\frac{\partial \phi}{\partial z}(z) \cdot z,
$$

where $g: \mathbb{R} \times[0,2 \pi] \rightarrow \mathbb{C}^{\times}$sends $w \mapsto z=e^{w}$, and we are using the flat metric on $\mathbb{R} \times[0,2 \pi]$ and $\mathbb{C}=\mathbb{R} \times \tilde{\gamma}$. It follows that $\partial \phi / \partial z$ (and hence $\partial \phi_{0} / \partial z$ ) is bounded for $|z|$ large. Hence $\partial \phi_{0} / \partial z$ is constant and $\phi_{0}(z)=c_{1} z$. Similarly, $\phi_{\infty}(z)=c_{-1} z^{-1}$. We then conclude that $\phi(z)=c_{-1} z^{-1}+c_{0}+c_{1} z$. The image of $\phi$ contains a neighborhood of the point at infinity, which contradicts the finite Hofer energy condition of $F$.

The following proposition is analogous to [19, Proposition 27], and its proof only needs some minor changes.

Proposition 5.11 (Gradient bound for a single curve) Let $F:(\Sigma, j, \mathbf{m}) \rightarrow\left(\mathbb{R} \times M^{*}, J\right)$ be a finite energy holomorphic map with bounded $\tau \circ F$. Then

$$
\sup _{z \in \dot{\Sigma}} \rho(z)\|\nabla F(z)\|<+\infty,
$$

where $\rho$ denotes the injectivity radius of the complete, finite volume hyperbolic metric $h$ on $\dot{\Sigma}$ which is compatible with $j$ and $\|\nabla F(z)\|$ is measured with respect to $h$ on $\dot{\Sigma}$ and the Riemannian metric on $\mathbb{R} \times M^{*}$ defined in Equation (15).

Remark 5.12 Near a puncture, $\rho(z)\|\nabla F(z)\|$, calculated with respect to a complete, finite volume hyperbolic metric (ie a cusp), is commensurate to $\|\nabla F(z)\|$, calculated with respect to a flat metric on a half-cylinder.

Proof We argue by contradiction. Suppose there is a sequence $z_{n} \in \dot{\Sigma}$ such that

$$
\rho\left(z_{n}\right)\left\|\nabla F\left(z_{n}\right)\right\| \rightarrow \infty
$$

as $n \rightarrow \infty$. By passing to a subsequence we may assume that $z_{n}$ converges to a puncture in $\mathbf{m}$. Next, there exist holomorphic charts $\psi_{n}: \mathbb{D} \stackrel{\sim}{\rightarrow} \mathcal{D}_{n} \subset \dot{\Sigma}$ such that $\psi_{n}(0)=z_{n}$ and

$$
C_{1} \rho\left(z_{n}\right) \leq\left\|\nabla \psi_{n}(z)\right\| \leq C_{2} \rho\left(z_{n}\right)
$$

for all $z \in \mathbb{D}$. Here $C_{1}$ and $C_{2}$ are two positive constants that do not depend on $z_{n}$ and $\nabla$ is calculated with respect to the standard Euclidean metric on $\mathbb{D}$ and the hyperbolic metric on $\dot{\Sigma}$. (This follows from Remark 5.12.) Setting

$$
\tilde{F}_{n}=\left(\tilde{a}_{n}, \tilde{f}_{n}\right)=\left(a \circ \psi_{n}, f \circ \psi_{n}\right),
$$

we have $\left\|\nabla \tilde{F}_{n}(0)\right\| \rightarrow+\infty$ as $n \rightarrow+\infty$. 
We now apply Lemma 5.9 to obtain the bubbling off of a finite energy plane $\widetilde{F}^{0}=$ $\left(\widetilde{a}^{0}, \tilde{f}^{0}\right)$. In both Cases (1) and (2) of Lemma 5.9, we have

$$
0 \leq \int_{\mathbb{C}}\left(\tilde{f}^{0}\right)^{*} d \alpha \leq \lim _{n \rightarrow \infty} \int_{\mathbb{D}}\left(\tilde{f}_{n}\right)^{*} d \alpha=\lim _{n \rightarrow \infty} \int_{\mathcal{D}_{n}} f^{*} d \alpha=0,
$$

because the size of $\mathcal{D}_{n}$ is going to zero as $n$ goes to infinity. Moreover, $\left\|\nabla \widetilde{F}^{0}\right\|$ is bounded by construction. Hence $\widetilde{F}^{0}$ is a constant map by Lemma 5.10 . This contradicts the property that $\left\|\nabla \widetilde{F}_{n}^{0}(0)\right\|=1$ for all $n$.

\subsubsection{Bound on $b$ for a single curve}

Proposition 5.13 Let $F: \dot{\mathbb{D}}=\mathbb{D}-\{0\} \rightarrow\left(\mathbb{R} \times M^{*}, J\right)$ be a finite energy $J$-holomorphic map such that $\tau \circ F$ is bounded. Then $b=t \circ F$ is bounded.

Proof Let us rewrite $F$ as

$$
F=(a, f):[0, \infty) \times S^{1} \rightarrow\left(\mathbb{R} \times M^{*}, J\right),
$$

with coordinates $(r, \theta)$ for $[0, \infty) \times S^{1}$. Here we are using the flat metric on the half-cylinder and the metric on $\mathbb{R} \times M^{*}$ given by Equation (15). The gradient bound (Proposition 5.11) and Remark 5.12 imply a uniform bound on $\left|b(r, \theta)-b\left(r, \theta^{\prime}\right)\right|$ for all $r, \theta, \theta^{\prime}$.

Arguing by contradiction, suppose that $b$ is not bounded. Without loss of generality, we may assume that $\limsup _{r \rightarrow \infty} b(r, \theta)=\infty$. By the bound on $\|\nabla F\|$, there are increasing sequences $\kappa_{n} \rightarrow \infty$ and $r_{n}^{(i)} \rightarrow \infty, i=1,2,3,4$, such that

- $r_{n}^{(1)}<r_{n}^{(2)}<r_{n}^{(3)}<r_{n}^{(4)}$;

- $r_{n}^{(i+1)}-r_{n}^{(i)} \rightarrow \infty, i=1,2,3$;

- $b\left(r_{n}^{(i)}, 0\right)=i \kappa_{n}, i=1,2,3,4$;

- $b(r, \theta) \geq 1$ for all $(r, \theta) \in\left[r_{n}^{(1)}, r_{n}^{(4)}\right] \times S^{1}$, ie $f\left(\left[r_{n}^{(1)}, r_{n}^{(4)}\right] \times S^{1}\right)$ is contained in the Top.

Hence we may view $\left.F\right|_{\left[r_{n}^{(1)}, r_{n}^{(4)}\right] \times S^{1}}$ as a map

$$
F_{n}:\left[r_{n}^{(1)}, r_{n}^{(4)}\right] \times S^{1} \rightarrow\left(\mathbb{R} \times \mathbb{R} \times \widehat{R_{+}(\Gamma)}, J\right) .
$$

Modulo translations in the $r-, s-$ and $t$-directions, we can extract a convergent subsequence of $F_{n}$. However, we need to exercise some care in order to ensure that the limiting curve is nonconstant. 
First suppose that there is a constant $c>0$ such that

$$
\sup _{r \in\left[r_{n}^{(2)}, r_{n}^{(3)}\right]}\left\|\nabla F_{n}\right\| \geq c
$$

for all $n$. (Note that we still have an upper bound on $\left\|\nabla F_{n}\right\|$.) Then, after translating in the $r$-and $\theta$-directions and restricting the domain, we may view $F_{n}$ as

$$
\widetilde{F}_{n}:\left[-R_{n}, R_{n}\right] \times S^{1} \rightarrow\left(\mathbb{R} \times \mathbb{R} \times \widehat{R_{+}(\Gamma)}, J\right),
$$

where $\left\|\nabla \widetilde{F}_{n}(0,0)\right\|>c$ and $R_{n} \rightarrow \infty$. (Note that we are using $\left[r_{n}^{(2)}, r_{n}^{(3)}\right] \subset\left[r_{n}^{(1)}, r_{n}^{(4)}\right]$ to give ourselves extra room on both sides.) By our assumption that $\tau \circ F$ is bounded, we can pass to a subsequence so that after translating in the $s$-and $t$-directions, $\widetilde{F}_{n}$ converges in $C_{\mathrm{loc}}^{\infty}$ to

$$
\tilde{F}_{\infty}: \mathbb{R} \times S^{1} \rightarrow\left(\mathbb{R} \times \mathbb{R} \times \widehat{R_{+}(\Gamma)}, J\right) .
$$

Since $\left\|\nabla \widetilde{F}_{\infty}(0,0)\right\| \geq c$, it follows that $\widetilde{F}_{\infty}$ is nonconstant. Also $\left\|\nabla \widetilde{F}_{\infty}\right\|$ is bounded by construction. Since $\widetilde{F}_{\infty}$ has zero $d \alpha$-energy as argued in Proposition 5.11, we can apply Lemma 5.10(2) to obtain a contradiction.

On the other hand, suppose there is a positive sequence $\varepsilon_{n} \rightarrow 0$ such that

$$
\sup _{r \in\left[r_{n}^{(2)}, r_{n}^{(3)}\right]}\left\|\nabla F_{n}\right\|=\varepsilon_{n} .
$$

By shrinking the interval $\left[r_{n}^{(2)}, r_{n}^{(3)}\right]$ if necessary, we may assume that the distance between $F_{n}\left(r_{n}^{(2)}, 0\right)$ and $F_{n}\left(r_{n}^{(3)}, 0\right)$ is 1 and the diameter of $Z_{n}=F_{n}\left(\left[r_{n}^{(2)}, r_{n}^{(3)}\right] \times S^{1}\right)$ is between 1 and 2. Such "long and thin" tubes in $\mathbb{R} \times \mathbb{R} \times \widehat{R_{+}(\Gamma)}$ can be eliminated by the isoperimetric inequality and the monotonicity lemma. Here the area is calculated with respect to the metric given in Equation (15). More precisely, by the gradient bound, $\gamma_{n}^{(2)}=F_{n}\left(\left\{r=r_{n}^{(2)}\right\}\right)$ has length $\leq 2 \pi \varepsilon_{n}$. Now recall the following wellknown isoperimetric inequality (see for example [23, Proposition A.1]):

Lemma 5.14 (Isoperimetric inequality) Let $(M, g)$ be a Riemannian manifold with bounded geometry. Then there exist constants $\varepsilon>0$ and $C>0$ satisfying the following: for every $0<r<\varepsilon$ and geodesic ball $B_{r}(x)$ of radius $r$, if $\gamma$ is a closed curve in $B_{r}(x)$ of length $l(\gamma)$, then there is a surface $S \subset B_{r}(x)$ with boundary $\gamma$ such that

$$
\operatorname{Area}(S) \leq C(l(\gamma))^{2} .
$$

Here the area and length are calculated with respect to the metric $g$. 
Continuing the proof of Proposition 5.13: by the isoperimetric inequality, there is a surface $S_{n}^{(2)}$ which bounds $\gamma_{n}^{(2)}$ and has area $\leq K_{0} \varepsilon_{n}^{2}$, where $K_{0}$ does not depend on $n$. The same can be said about $\gamma_{n}^{(3)}=F_{n}\left(\left\{r=r_{n}^{(3)}\right\}\right)$.

We now claim that

$$
C \cdot \operatorname{Area}\left(Z_{n}\right) \leq \operatorname{Area}\left(S_{n}^{(2)} \cup S_{n}^{(3)}\right) \leq 2 K_{0} \varepsilon_{n}^{2},
$$

for some positive constant $C$ which is independent of $n$. The first inequality follows from noting that

(i) $C_{1} \cdot \int_{S} \omega \leq \operatorname{Area}(S)$ for any surface $S$ (Wirtinger's inequality);

(ii) $\int_{Z_{n}} \omega=\int_{S_{n}^{(2)} \cup S_{n}^{(3)}} \omega$ (since $Z_{n} \cup S_{n}^{(2)} \cup S_{n}^{(3)}$ is nullhomologous due to the fact that $Z_{n}$ is thin);

(iii) $C_{2} \cdot \operatorname{Area}\left(Z_{n}\right) \leq \int_{Z_{n}} \omega$ (since $J$ tames $\omega$ and $Z_{n}$ is holomorphic).

Here $\omega=d\left(e^{s} \alpha\right)$ is the symplectization 2-form and (i) and (iii) work because each $Z_{n}$, after translation, is contained in $0 \leq s \leq 2$ by the diameter bound.

On the other hand, since $\varepsilon_{n} \rightarrow 0$ and the distance between $F_{n}\left(r_{n}^{(2)}, 0\right)$ and $F_{n}\left(r_{n}^{(3)}, 0\right)$ is fixed, there is a constant $\delta>0$, independent of $n$, such that a ball $B_{\delta}\left(x_{n}\right)$ of radius $\delta$ centered at some point $x_{n} \in Z_{n}$ does not intersect the boundary of $Z_{n}$. Then by the monotonicity lemma,

$$
\operatorname{Area}\left(Z_{n} \cap B_{\delta}\left(x_{n}\right)\right) \geq K_{1} \delta^{2}
$$

for some constant $K_{1}>0$ which is independent of $n$. This contradicts Inequality (16) for sufficiently small $\varepsilon_{n}$. This concludes the proof of Proposition 5.13.

5.5.3 Removal of singularities We now state some corollaries of the bound on the $t$-coordinate.

Corollary 5.15 (Removal of singularities for Top/Bottom) Every finite energy holomorphic map

$$
F=(a, f, v): \dot{\mathbb{D}}=\{z \in \mathbb{C}|0<| z \mid<1\} \rightarrow\left(\mathbb{R} \times \mathbb{R} \times \widehat{R_{+}(\Gamma)}, J\right)
$$

with $\tau \circ f$ bounded, extends to a finite energy holomorphic map

$$
\bar{F}: \mathbb{D} \rightarrow\left(\mathbb{R} \times \mathbb{R} \times \widehat{R_{+}(\Gamma)}, J\right) .
$$

Proof Since $b$ is bounded by Proposition 5.13, the usual argument for a symplectization applies: either $F$ approaches a closed orbit of the Reeb vector field as $|z| \rightarrow 0$, or the singularity is removable. There are no closed orbits on $\mathbb{R} \times \mathbb{R} \times \widehat{R_{+}(\Gamma)}$, so the result follows. 
Corollary 5.16 Let $F=(a, f):(\Sigma, j, \mathbf{m}) \rightarrow\left(\mathbb{R} \times M^{*}, J\right)$ be a finite energy $J-$ holomorphic map with $\tau \circ F$ bounded. Then the set of punctures $\mathbf{m}$ can be written as $\mathbf{m}^{+} \sqcup \mathbf{m}^{-} \sqcup \mathbf{m}^{r}$, where

- for any $z_{+} \in \mathbf{m}^{+}$we have $\lim _{z \rightarrow z_{+}} a(z)=+\infty$ and $\limsup _{z \rightarrow z_{+}}|b(z)|<+\infty$;

- for any $z_{-} \in \mathbf{m}^{-}$we have $\lim _{z \rightarrow z_{-}} a(z)=-\infty$ and $\limsup _{z \rightarrow z_{-}}|b(z)|<+\infty$;

- for any $z_{r} \in \mathbf{m}^{r}$ the singularity is removable.

\subsection{Bounds for sequences of holomorphic curves}

To extend the SFT and ECH compactness theorems to our situation, we need uniform bounds on the $t$-coordinate for sequences of holomorphic curves.

5.6.1 Gradient bound for a sequence We start with the following lemma which gives a gradient bound for a sequence of holomorphic maps. The proof is similar to the proof of Proposition 5.11 and to [3, Section 10.2.1].

Lemma 5.17 Let $F_{n}=\left(a_{n}, f_{n}\right):\left(\Sigma_{n}, j_{n}, \mathbf{m}_{n}\right) \rightarrow\left(\mathbb{R} \times M^{*}, J\right)$ be a sequence of $J$-holomorphic maps such that there exists $C>0$ with $E\left(F_{n}\right)<C$ and $\left|\tau \circ F_{n}\right|<C$. Then we can remove finite sets $\mathbf{m}_{n}^{0}$ from $\Sigma_{n} \backslash \mathbf{m}_{n}$ so that the sequence

$$
F_{n}:\left(\Sigma_{n} \backslash\left(\mathbf{m}_{n} \cup \mathbf{m}_{n}^{0}\right), j_{n}\right) \rightarrow\left(\mathbb{R} \times M^{*}, J\right)
$$

satisfies the bound

$$
\rho_{n}(x)\left\|\nabla F_{n}(x)\right\| \leq C, \quad \forall x \in \Sigma_{n} \backslash\left(\mathbf{m}_{n} \cup \mathbf{m}_{n}^{0}\right),
$$

where the norm of gradient is computed with respect to the unique complete, finite volume hyperbolic metric which is compatible with $j_{n}$ on $\Sigma_{n} \backslash\left(\mathbf{m}_{n} \cup \mathbf{m}_{n}^{0}\right)$, and with respect to the metric on $\mathbb{R} \times M^{*}$ given by Equation (15). Here the cardinality $\left|\mathbf{m}_{n}^{0}\right|$ is independent of $n$.

Proof Suppose there is a sequence $z_{n} \in \Sigma_{n} \backslash \mathbf{m}_{n}$ such that $\rho_{n}\left(z_{n}\right)\left\|\nabla F_{n}\left(z_{n}\right)\right\| \rightarrow \infty$ for $n \rightarrow \infty$. There exist holomorphic charts $\psi_{n}: \mathbb{D} \stackrel{\sim}{\rightarrow} \mathcal{D}_{n} \subset \Sigma_{n} \backslash \mathbf{m}_{n}$ such that $\psi_{n}(0)=z_{n}$ and

$$
C_{1} \rho_{n}\left(z_{n}\right) \leq\left\|\nabla \psi_{n}(z)\right\| \leq C_{2} \rho_{n}\left(z_{n}\right)
$$

for all $z \in \mathbb{D}$. Here $C_{1}$ and $C_{2}$ are two positive constants that do not depend on $z_{n}$ and $\nabla$ is calculated with respect to the standard Euclidean metric on $\mathbb{D}$ and the complete hyperbolic metric on $\Sigma_{n} \backslash \mathbf{m}_{n}$. Setting

$$
\widetilde{F}_{n}=\left(\widetilde{a}_{n}, \tilde{f}_{n}\right)=\left(a_{n} \circ \psi_{n}, f_{n} \circ \psi_{n}\right),
$$

we have $\left\|\nabla \widetilde{F}_{n}(0)\right\| \rightarrow+\infty$ as $n \rightarrow+\infty$. 
By Lemma 5.9 we obtain the bubbling off of a nonconstant finite energy plane $\widetilde{F}^{0}: \mathbb{C} \rightarrow\left(\mathbb{R} \times M^{*}, J\right)$ or $\widetilde{F}^{0}: \mathbb{C} \rightarrow\left(\mathbb{R} \times \mathbb{R} \times \widehat{R_{ \pm}(\Gamma)}, J\right)$. The latter cannot happen because a nonconstant finite energy plane in $\mathbb{R} \times \mathbb{R} \times \widehat{R_{ \pm}(\Gamma)}$ would extend to a nonconstant holomorphic sphere by Corollary 5.15. (Note that there are no closed orbits in $\mathbb{R} \times \widehat{R_{ \pm}(\Gamma)}$.) This is a contradiction since the symplectic form on $\mathbb{R} \times \mathbb{R} \times \widehat{R_{ \pm}(\Gamma)}$ is exact. Also observe that the finite energy plane $\widetilde{F}^{0}$ is positively asymptotic to a closed Reeb orbit because there are no nonconstant holomorphic spheres in $\left(\mathbb{R} \times M^{*}, J\right)$.

In order to achieve the gradient bound given by Equation (18), we add marked points in the bubbling neighborhoods as in [3, Subsection 10.2.1]. Since there is a uniform lower bound on the areas of finite energy planes, we only need a finite set $\mathbf{m}_{n}^{0}$.

5.6.2 Bound on $b_{n}$, assuming topological complexity bound We now prove the following bound on $b_{n}$, provided we have bounds on the energy and genus (and number of marked points).

Proposition 5.18 Let $F_{n}=\left(a_{n}, f_{n}\right):\left(\Sigma_{n}, j_{n}, \mathbf{m}_{n}\right) \rightarrow\left(\mathbb{R} \times M^{*}, J\right)$ be a sequence of holomorphic maps with uniform upper bounds on $\left|\tau \circ F_{n}\right|$, the energy $E\left(F_{n}\right)$, and the "topological complexity" $g\left(\Sigma_{n}\right)+\left|\mathbf{m}_{n}\right|$. Then there is a uniform upper bound on $\left|b_{n}\right|=\left|t \circ f_{n}\right|$.

Proof Let $F_{n}$ be a sequence as in the hypothesis of Proposition 5.18. Arguing by contradiction, suppose the functions $b_{n}$ are not uniformly bounded. Without loss of generality we may assume that $\lim _{n \rightarrow \infty}\left(\sup _{\dot{\Sigma}_{n}} b_{n}\right)=+\infty$ for $n \rightarrow \infty$. By Lemma 5.17 we can add marked points $\mathbf{m}_{n}^{0}$ to $\dot{\Sigma}_{n}=\Sigma_{n} \backslash \mathbf{m}_{n}$ to obtain the gradient bound given by Equation (18) for the sequence $F_{n}$.

By Theorem 5.2, there is a subsequence of $\mathbf{S}_{n}^{\prime}=\left(\Sigma_{n}, j_{n}, \mathbf{m}_{n} \cup \mathbf{m}_{n}^{0}\right)$ which converges to a nodal surface $\mathbf{S}=(\Sigma, j, \mathbf{m}, D)$. Fix $\epsilon<\frac{1}{4} \log (1+\sqrt{2})$ (ie $1 / 4$ of the constant required for the thick-thin decomposition), and consider the cover

$$
\Sigma-(\mathbf{m} \cup D)=C_{0} \cup \ldots \cup C_{k},
$$

where $C_{i}$ is either a connected component of $\operatorname{Thick}_{2 \epsilon}(\mathbf{S})$ or a "connected component" of $\operatorname{Thin}_{3 \epsilon}(\mathbf{S})$. (Here any two components of $\operatorname{Thin}_{3 \epsilon}(\mathbf{S})$, whose corresponding marked points in $D$ are identified, are regarded as part of the same "connected component" of $\operatorname{Thin}_{3 \epsilon}(\mathbf{S})$.) Similarly consider the cover

$$
\Sigma_{n}-\left(\mathbf{m}_{n} \cup \mathbf{m}_{n}^{0}\right)=C_{0}^{n} \cup \ldots \cup C_{k}^{n},
$$

where $C_{i}^{n}$ is a component of $\operatorname{Thick}_{\epsilon}\left(\mathbf{S}_{n}^{\prime}\right)$ or a "connected component" of $\operatorname{Thin}_{4 \epsilon}\left(\mathbf{S}_{n}^{\prime}\right)$, and $C_{i}^{n}$ corresponds to $C_{i}$. By Proposition 5.4, for sufficiently large $n, C_{i}$ is contained in a component $C_{i}^{n}$ for all $i$. 
Now define

$$
\Delta_{n}\left(C_{i}^{n}\right)=\sup _{C_{i}^{n}} b_{n}-\inf _{C_{i}^{n}} b_{n}
$$

Since $\lim _{n \rightarrow \infty}\left(\sup _{\dot{\Sigma}_{n}} b_{n}\right)=+\infty$ and the ends of $F_{n}$ are asymptotic to cylinders over Reeb orbits in $M$, it follows that

$$
\lim _{n \rightarrow \infty}\left(\sup _{\dot{\Sigma}_{n}} b_{n}-\inf _{\dot{\Sigma}_{n}} b_{n}\right)=+\infty
$$

Now, since each covering has the same finite number of components, there must be one - which we call $C_{0}^{n}$ without loss of generality - for which $\lim _{n \rightarrow \infty} \Delta_{n}\left(C_{0}^{n}\right)=+\infty$. By Lemma 5.17 and Proposition 5.4, $\left\|\nabla F_{n}\right\|$ is uniformly bounded on Thick $2 \epsilon(\mathbf{S})$. Since the variation of $b_{n}$ is bounded on the thick part due to a bound on the diameter, $C_{0}$ must be a connected component of $\operatorname{Thin}_{3 \epsilon}(\mathbf{S})$.

By reparametrizing the component $C_{0}^{n}$ using a standard flat cylinder, we can write $F_{n}$ on $C_{0}^{n}$ as

$$
F_{n}:\left[0, r_{n}\right] \times S^{1} \rightarrow\left(\mathbb{R} \times \mathbb{R} \times \widehat{R_{+}(\Gamma)}, J\right),
$$

where $\left\|\nabla F_{n}\right\|$ is uniformly bounded by Lemma 5.17, in view of Remark 5.12. This uniform bound implies that $\operatorname{Im} F_{n}$ has bounded diameter (independent of $n$ ) when restricted to any circle $\{r=$ const $\}$.

The rest of the proof is as in Proposition 5.13. There exist $\kappa_{n} \rightarrow \infty$ and $r_{n}^{(i)} \rightarrow \infty$, $i=1,2,3,4$, such that

- $0<r_{n}^{(1)}<r_{n}^{(2)}<r_{n}^{(3)}<r_{n}^{(4)}<r_{n}$;

- $r_{n}^{(i+1)}-r_{n}^{(i)} \rightarrow \infty, i=1,2,3$;

- $b_{n}\left(r_{n}^{(i+1)}, 0\right)-b_{n}\left(r_{n}^{(i)}, 0\right)=\kappa_{n}, i=1,2,3 ;{ }^{6}$

- $b_{n}(r, \theta) \geq 1$ for all $(r, \theta) \in\left[r_{n}^{(1)}, r_{n}^{(4)}\right] \times S^{1}$, ie $f\left(\left[r_{n}^{(1)}, r_{n}^{(4)}\right] \times S^{1}\right)$ is contained in the Top.

If $\sup \left\|\nabla F_{n}\right\|$ is bounded below by $c>0$ on $\left[r_{n}^{(2)}, r_{n}^{(3)}\right] \times S^{1}$, then, after restricting the domain of $F_{n}$ and translating in the $r$-and $\theta$-directions, we obtain

$$
\widetilde{F}_{n}:\left[-R_{n}, R_{n}\right] \times S^{1} \rightarrow\left(\mathbb{R} \times \mathbb{R} \times \widehat{R_{+}(\Gamma)}, J\right),
$$

where $\left\|\nabla \widetilde{F}_{n}(0,0)\right\| \geq c$ and $R_{n} \rightarrow \infty$. The limit curve

$$
\widetilde{F}_{\infty}: \mathbb{R} \times S^{1} \rightarrow\left(\mathbb{R} \times \mathbb{R} \times \widehat{R_{+}(\Gamma)}, J\right),
$$

${ }^{6}$ Note that, unlike the corresponding condition for Proposition 5.13, we are taking the difference of the $b_{n}$ values. 
is a nonconstant holomorphic curve. By Corollary 5.15, we can extend this function to a nonconstant holomorphic sphere in $\left(\mathbb{R} \times \mathbb{R} \times \widehat{R_{+}(\Gamma)}, J\right)$ and obtain a contradiction. On the other hand, if sup $\left\|\nabla F_{n}\right\| \rightarrow 0$ on $\left[r_{n}^{(2)}, r_{n}^{(3)}\right] \times S^{1}$, then we can eliminate the "long and thin" tubes in $\mathbb{R} \times \mathbb{R} \times \widehat{R_{+}(\Gamma)}$ as in Proposition 5.13.

Corollary 5.19 Let $M^{*}$ be the completion of a sutured contact manifold $M$ and let $J$ be a tailored almost complex structure on the symplectization $\mathbb{R} \times M^{*}$, as usual. Then the SFT compactness theorem [3, Theorem 10.1] holds for $J$-holomorphic curves in $\mathbb{R} \times M^{*}$ whose punctures are asymptotic to Reeb orbits.

Proof We need to show that any sequence in $\mathcal{M}_{g}\left(\underline{\gamma} ; \underline{\gamma^{\prime}} ; J\right)$ has a subsequence which converges to a holomorphic building in the sense of [3]. By Lemma 5.5, there is a uniform upper bound on $\tau$ for the curves in the sequence. By [3, Proposition 5.13] there is a uniform upper bound on the Hofer energy of the curves in the sequence. By Proposition 5.18 there is then a uniform upper bound on $t$. Thus the projections of all the holomorphic curves in the sequence to $M^{*}$ are contained in a compact set, and the rest of the argument in [3] carries over.

5.6.3 Bound on $b_{n}$ in dimension four We turn now to the compactness theorem for $\mathrm{ECH}$. For this purpose we will prove the bound on $b_{n}$ without any constraints on the genus, but assuming that $\mathbb{R} \times M^{*}$ has dimension four. The proof is based on a version of Gromov compactness due to Taubes which uses currents and does not assume any genus bounds; see [35, Proposition 3.3; 24, Lemma 9.8].

We recall some basic terminology from ECH. An orbit set is a finite set of pairs $\left\{\left(\gamma_{i}, m_{i}\right)\right\}$, where the $\gamma_{i}$ are distinct embedded Reeb orbits, and the $m_{i}$ are positive integers. In the terminology of [24], a flow line from the orbit set $\left\{\left(\gamma_{i}, m_{i}\right)\right\}$ to the orbit set $\left\{\left(\gamma_{j}^{\prime}, m_{j}^{\prime}\right)\right\}$ is a finite energy holomorphic curve $F:(\Sigma, j, \mathbf{m}) \rightarrow\left(\mathbb{R} \times M^{*}, J\right)$ such that

(1) $F$ is an embedding, except perhaps for repeated $\mathbb{R}$-invariant cylinders which do not intersect the other components of $F$;

(2) $F$ has positive punctures at covers of $\gamma_{i}$ with total multiplicity $m_{i}$, negative punctures at covers of $\gamma_{j}^{\prime}$ with total multiplicity $m_{j}^{\prime}$, and no other punctures.

Proposition 5.20 Suppose $\operatorname{dim}\left(\mathbb{R} \times M^{*}\right)=4$. Let $F_{n}=\left(a_{n}, f_{n}\right):\left(\Sigma_{n}, j_{n}, \mathbf{m}_{n}\right) \rightarrow$ $\left(\mathbb{R} \times M^{*}, J\right)$ be a sequence of flow lines from $\left\{\left(\gamma_{i}, m_{i}\right)\right\}$ to $\left\{\left(\gamma_{j}^{\prime}, m_{j}^{\prime}\right)\right\}$. Then there are uniform upper bounds on $\left|\tau \circ f_{n}\right|$ and $\left|b_{n}\right|=\left|t \circ f_{n}\right|$. 
Proof The bound on $\tau$ follows from Lemma 5.5. To prove the bound on $t$, suppose on the contrary that there is a sequence of flow lines $F_{n}$ with $b_{n}$ unbounded. Without loss of generality there exist $x_{n} \in \dot{\Sigma}_{n}$ such that $b_{n}\left(x_{n}\right) \rightarrow+\infty$. Now consider the restriction

$$
F_{n}^{\prime}: \Sigma_{n}^{\prime} \rightarrow \mathbb{R} \times[1, \infty) \times \widehat{R_{+}(\Gamma)}
$$

of $F_{n}$ where

$$
\Sigma_{n}^{\prime}=\left\{x \in \dot{\Sigma}_{n} \mid f_{n}(x) \in[1, \infty) \times \widehat{R_{+}(\Gamma)}\right\} .
$$

Let $C_{n}^{\prime}$ be the holomorphic subvariety obtained by translating $F_{n}^{\prime}\left(\Sigma_{n}^{\prime}\right)$ by $a_{n}\left(x_{n}\right)$ in the $s$-direction and by $b_{n}\left(x_{n}\right)$ in the $t$-direction. (From now on, we will not distinguish between holomorphic maps and their images, viewed as currents.) We then set

$$
C_{n}=C_{n}^{\prime} \cap\left(\left[-c_{n}, c_{n}\right] \times\left[-d_{n}, d_{n}\right] \times \widehat{R_{+}(\Gamma)}\right),
$$

where $c_{n}, d_{n} \rightarrow \infty$ and $0<d_{n} \ll b_{n}\left(x_{n}\right)$. Note that $C_{n}$ passes through $\{(0,0)\} \times \widehat{R_{+}(\Gamma)}$. We may assume without loss of generality that $\int_{C_{n}} d \alpha^{*} \rightarrow 0$.

By the Gromov compactness theorem via currents [35, Proposition 3.3], we can pass to a subsequence so that $C_{n}$ converges weakly as currents in $\left(\mathbb{R} \times \mathbb{R} \times \widehat{R_{+}(\Gamma)}, J\right)$ to a proper $J$-holomorphic subvariety $C$, so that, for any compact set $K \subset \mathbb{R} \times \mathbb{R} \times \widehat{R_{+}(\Gamma)}$,

$$
\sup _{x \in C_{n} \cap K} \operatorname{dist}(x, C)+\sup _{x \in C \cap K} \operatorname{dist}\left(x, C_{n}\right) \rightarrow 0,
$$

as $n \rightarrow \infty$. More precisely, for any compact set $K \subset \mathbb{R} \times \mathbb{R} \times \widehat{R_{+}(\Gamma)}$, we can pass to a subsequence so that the intersections of the curves $C_{n}$ with $K$ converge to a $J$-holomorphic subvariety in $K$, using the fact that there is a uniform upper bound on the integral of the symplectic form $d\left(e^{s} \alpha^{*}\right)$ over $C_{n} \cap K$. An exhaustion argument then gives a subsequence converging on all of $\mathbb{R} \times \mathbb{R} \times \widehat{R_{+}(\Gamma)}$ as above.

We claim now that $\left.d \alpha^{*}\right|_{C}=0$. To see this, let $p \in C$ and let $\varphi: \mathbb{R} \times \mathbb{R} \times \widehat{R_{+}(\Gamma)} \rightarrow[0,1]$ be a compactly supported smooth function with $\varphi(p)=1$. Since $\int_{C_{n}} d \alpha^{*} \rightarrow 0$ and $\left.d \alpha^{*}\right|_{C_{n}} \geq 0$ on all of $C_{n}$, we have $\int_{C_{n}} \varphi d \alpha^{*} \rightarrow 0$. Since $C_{n}$ converges to $C$ as functionals on compactly supported 2 -forms, we obtain $\int_{C} \varphi d \alpha^{*}=0$. Since $\left.d \alpha^{*}\right|_{C} \geq 0$ on all of $C$, we conclude that $\left.d \alpha^{*}\right|_{C}$ vanishes on a neighborhood of $p$. This proves the claim.

It follows now that $C$ is supported on $\mathbb{R} \times \gamma$, where $\gamma$ is a Reeb orbit. Note that $\gamma$ is not a closed orbit, and instead is a line. Now $C$ covers all of $\mathbb{R} \times \gamma$ by the properness of $C$, and the fact that holomorphic maps are open. On the other hand, $\mathbb{R} \times \gamma$ has infinite Hofer energy, while there is a uniform upper bound on the Hofer energy of $C_{n}$ by [3, Proposition 5.13]. This contradicts the weak convergence of $C_{n}$ to $C$. 
Corollary 5.21 Suppose $\operatorname{dim}\left(\mathbb{R} \times M^{*}\right)=4$. Then the ECH compactness theorem [24, Lemma 9.8] holds for $J$-holomorphic curves in the symplectization of the completion of a sutured contact manifold, provided that we choose the almost complex structure $J$ on $\mathbb{R} \times M^{*}$ to be tailored to $\left(M^{*}, \alpha^{*}\right)$ in the sense of Section 3.1.

\section{Definition of the sutured contact homology and sutured ECH}

We now use the compactness theorems established in the previous section to define the sutured contact homology and sutured ECH groups and to prove Theorem 1.1.

\subsection{Definition of sutured contact homology}

Let $(M, \Gamma, U(\Gamma), \xi)$ be a sutured contact manifold and $\alpha$ be an adapted contact form for $\xi$. Let $\left(M^{*}, \alpha^{*}\right)$ be the completion of $(M, \alpha)$ and $J$ be an almost complex structure on $\mathbb{R} \times M^{*}$ which is tailored to $\left(M^{*}, \alpha^{*}\right)$. Since all the periodic orbits of $R_{\alpha^{*}}$ are contained in $M$, by performing a small perturbation of $\alpha^{*}$ supported in $M$ we may assume that $\alpha^{*}$ is nondegenerate, ie all the periodic orbits of $R_{\alpha^{*}}$ are nondegenerate.

We define the sutured contact homology $\operatorname{HC}(M, \Gamma, \alpha, J)$ to be the contact homology of $\left(M^{*}, \alpha^{*}, J\right)$ as follows: A periodic orbit of the Reeb vector field $R_{\alpha^{*}}$ is said to be good if it does not cover a simple orbit $\gamma$ an even number of times, where the first return map $\xi_{\gamma(0)} \rightarrow \xi_{\gamma(T)}$ has an odd number of eigenvalues in the interval $(-1,0)$. Let $\mathcal{P}(\alpha)$ be the set of good periodic orbits $\gamma$ of $R_{\alpha^{*}}$. The contact homology chain complex $\mathcal{A}(\alpha, J)$ is the free supercommutative $\mathbb{Q}$-algebra with unit generated by elements of $\mathcal{P}(\alpha)$, where the grading and the boundary map $\partial \gamma$ are defined in the usual way (as in [11]) with respect to the $\alpha^{*}$-adapted almost complex structure $J$. The homology of $\mathcal{A}(\alpha, J)$ is the sutured contact homology algebra $H C(M, \Gamma, \alpha, J)$.

It follows from Corollary 5.19 that the necessary Gromov compactness holds to show that the differential $\partial$ is well-defined and $\partial^{2}=0$. Namely, if $\gamma$ is a periodic orbit, then there are only finitely many collections of negative ends with total action less than that of $\gamma$. Hence $\partial \gamma$ counts holomorphic curves in the quotients by the $\mathbb{R}$-action of index 1 moduli spaces $\mathcal{M}_{0}\left(\gamma ; \gamma_{1}^{\prime}, \ldots, \gamma_{l}^{\prime}\right)$, where we range over finitely many $\left(\gamma_{1}^{\prime}, \ldots, \gamma_{l}^{\prime}\right)$. If these moduli spaces are cut out transversely, then it follows from Corollary 5.19 that $\partial \gamma$ is a finite count of holomorphic curves. Similarly, the proof that $\partial^{2}=0$ involves considering the boundaries of quotients by the $\mathbb{R}$-action of index 2 moduli spaces $\mathcal{M}=\mathcal{M}_{0}\left(\gamma ; \gamma_{1}^{\prime}, \ldots, \gamma_{l}^{\prime}\right)$, where for any given $\gamma$ there are only finitely many possibilities for $\gamma^{\prime}$. If these moduli spaces are cut out transversely, then it follows from Corollary 5.19 that $\partial^{2}$ counts points in the boundary of a compact 1 -manifold. 
Disclaimer Already for closed contact manifolds, it is usually not possible to choose $J$ so that all of the above moduli spaces are cut out transversely. This problem arises because of multiply covered holomorphic curves of negative index. Thus in general the differential $\partial$ needs to be defined as a count of points in some abstract perturbation of the (compactified) moduli space of index 1 holomorphic curves. Even in a lucky situation where all relevant moduli spaces of holomorphic curves are cut out transversely, one still needs some abstract perturbations to define the chain homotopies needed to prove that the contact homology is independent of the choice of contact form and almost complex structure. This problem arises because in a generic 1-parameter family of data there can be holomorphic buildings with repeated index -1 curves.

The necessary abstract perturbations to solve the above problems in the closed case are a work in progress by Hofer, Wysocki and Zehnder (see [20] for an overview), and are expected to carry over directly to the sutured case. But strictly speaking Theorem 1.1 should be regarded as a conjecture until this work has been completed.

On the other hand, transversality for somewhere injective holomorphic curves in $\mathcal{M}_{g}\left(\underline{\gamma} ; \underline{\gamma}^{\prime} ; J\right)$ can be achieved by taking $J$ to be generic inside $M$, while keeping it tailored. In fact, the transversality argument in [9] carries over directly to the sutured case. In particular, it suffices to perturb $J$ arbitrarily near the periodic orbits in order to attain transversality for somewhere injective curves.

\subsection{Invariance of the contact homology algebra}

Modulo the above disclaimers, we now prove the following proposition, which will complete the proof of Theorem 1.1(1). Below we suppress the (not yet defined) abstract perturbations from the discussion.

Proposition 6.1 Let $(M, \Gamma, \xi)$ be a sutured contact manifold.

(1) The contact homology algebra $H C(M, \Gamma, \alpha, J)$ does not depend on the choice of adapted contact form $\alpha$ with $\operatorname{Ker}(\alpha)=\xi$ or tailored almost complex structure $J$, and so we can denote it by $H C(M, \Gamma, \xi)$.

(2) More generally, a one-parameter family of contact structures $\left\{\xi_{t} \mid t \in[0,1]\right\}$ which are the kernels of a one-parameter family $\left\{\alpha^{\lambda} \mid \lambda \in[0,1]\right\}$ of adapted contact forms on $(M, \Gamma, U(\Gamma))$ induces an isomorphism $H C\left(M, \Gamma, \xi^{0}\right) \simeq H C\left(M, \Gamma, \xi^{1}\right)$ which depends only on the homotopy class of the path $\left\{\xi_{t}\right\}$.

Proof Let $\alpha^{0}$ and $\alpha^{1}$ be two contact 1 -forms which are adapted to $(M, \Gamma, U(\Gamma))$, and are connected by a 1 -parameter family $\alpha^{\lambda}, \lambda \in[0,1]$, of adapted contact 1forms; also let $\left(\alpha^{\lambda}\right)^{*}$ be the completion of $\alpha^{\lambda}$ to $M^{*}$. Note that we are not assuming 
that $\operatorname{ker} \alpha^{0}=\operatorname{ker} \alpha^{1}$, only that they are isotopic. Let $J^{\lambda}, \lambda \in[0,1]$, be an almost complex structure on $\mathbb{R} \times M^{*}$ which is tailored to $\left(M^{*},\left(\alpha^{\lambda}\right)^{*}\right)$. In particular, the projection $J_{0}^{\lambda}$ of $J^{\lambda}$ to $\left(\widehat{R_{ \pm}(\Gamma)}, \widehat{\beta}_{ \pm}^{\lambda}\right)$ is $\hat{\beta}_{ \pm}^{\lambda}$-adapted. Here $\widehat{\beta}_{ \pm}^{\lambda}$ is the completion of the Liouville 1-form $\left.\alpha^{\lambda}\right|_{R_{ \pm}(\Gamma)}$ so that the Liouville vector field $Y^{\lambda}=\partial_{\tau}$ for $\tau \geq 0$; let us also write $\left(\beta_{ \pm}^{\lambda}\right)_{0}$ for the restriction of $\hat{\beta}_{ \pm}^{\lambda}$ to $\partial R_{ \pm}(\Gamma)$. We now define an isomorphism $H C\left(M, \Gamma, \alpha^{0}, J^{0}\right) \stackrel{\simeq}{\rightarrow} H C\left(M, \Gamma, \alpha^{1}, J^{1}\right)$.

Step 1 First consider the case when $\hat{\beta}_{ \pm}^{\lambda}$ and $J_{0}^{\lambda}$ are independent of $\lambda$ on the region where $\tau \geq 0$. We then define a chain map

$$
\Phi: \mathcal{A}\left(\alpha^{0}, J^{0}\right) \rightarrow \mathcal{A}\left(\alpha^{1}, J^{1}\right) .
$$

as follows. Let $\phi: \mathbb{R} \rightarrow[0,1]$ be a smooth nonincreasing function with $\phi(s)=1$ for $s \leq-N$ and $\phi(s)=0$ for $s \geq N$, where $N \gg 0$. On $\mathbb{R} \times M^{*}$ with coordinates $(s, y)$, define the almost complex structure $\widetilde{J}$ so that $\widetilde{J}(s, y)=J^{\phi(s)}(s, y)$. Let $\mathcal{M}_{g}\left(\underline{\gamma} ; \underline{\gamma^{\prime}} ; \widetilde{J}\right)$ be the moduli space of genus $g$ finite energy holomorphic maps $F:(\Sigma, \bar{j}, \overline{\mathbf{m}}) \rightarrow$ $\left(\mathbb{R} \times M^{*}, \widetilde{J}\right)$ with positive ends $\gamma$ which are periodic orbits of $R_{\left(\alpha^{0}\right)^{*}}$ and negative ends $\gamma^{\prime}$ which are periodic orbits of $R_{\left(\alpha^{1}\right)^{*}}$. Then the chain map $\Phi(\gamma)$ counts elements of index zero moduli spaces $\mathcal{M}=\mathcal{M}_{0}\left(\gamma ; \gamma_{1}^{\prime}, \ldots, \gamma_{k}^{\prime} ; \widetilde{J}\right)$. Note that the almost complex structure $\widetilde{J}$ is tamed by the symplectic form $d\left(e^{s} \alpha^{\phi(s)}\right)$, provided $|d \phi / d s|$ is sufficiently small for all $s$. Moreover, $\widetilde{J}$ is $\alpha^{0}$-adapted for $s \geq N$ and $\alpha^{1}$-adapted for $s \leq-N$.

We claim that all the curves in $\mathcal{M}_{0}(\gamma ; \ldots ; \widetilde{J})$, when projected to $M^{*}$, are contained inside a compact subset of $M^{*}$, so that they satisfy the Gromov compactness needed to show that $\Phi$ is a well-defined chain map. Since the projection $J_{0}^{\phi(s)}$ of $\widetilde{J}$ is $s-$ invariant on $\tau \geq 0$, it follows that no such curve enters the region $\tau \geq 0$. Now, if there is a sequence of curves $F_{n} \in \mathcal{M}_{0}(\gamma ; \ldots ; \widetilde{J})$ and $z_{n} \in \dot{\Sigma}$ such that $t \circ F_{n}\left(z_{n}\right) \rightarrow \infty$, then an argument similar to the proof of Proposition 5.18 implies the existence of a nonconstant finite energy holomorphic map to $\mathbb{R} \times \mathbb{R} \times R_{ \pm}(\Gamma)$, either with respect to $\widetilde{J}$ or with respect to $J^{0}$ or $J^{1}$. In any case, since there are no periodic orbits inside $\mathbb{R} \times \mathbb{R} \times R_{ \pm}(\Gamma)$, we have a contradiction.

Arguing as usual, we can prove that $\Phi$ has a homotopy inverse $\Psi$, so that $\Phi$ induces an isomorphism on homology.

Step 2 Next suppose that $J^{0}$ and $J^{1}$ do not agree on the ends $\tau \geq 0$. We define an intermediate almost complex structure $J^{2}$ together with a 1-form $\left(\alpha^{2}\right)^{*}$ on $M^{*}$ so that there are isomorphisms $H C\left(\alpha^{0}, J^{0}\right) \simeq H C\left(\left(\alpha^{2}\right)^{*}, J^{2}\right)$ and $H C\left(\left(\alpha^{2}\right)^{*}, J^{2}\right) \simeq$ $H C\left(\alpha^{1}, J^{1}\right)$.

The proof of Lemma 3.2 and Corollary 3.3 shows that there exist an almost complex structure $J_{0}^{2}$ and 1 -forms $\widehat{\beta}_{ \pm}^{2}$ on $\widehat{R_{ \pm}(\Gamma)}$ which satisfy the following: 
- Where $\tau \gg 0$, the 1 -form $\hat{\beta}_{ \pm}^{2}$ agrees with $\left(\beta_{ \pm}^{0}\right)_{0}$, and the almost complex structure $J_{0}^{2}$ is $\left(\beta_{ \pm}^{0}\right)_{0}$-adapted.

- Where $\tau \leq 0$ we have $J_{0}^{2}=J_{0}^{1}$ and $\widehat{\beta}_{ \pm}^{2}=\hat{\beta}_{ \pm}^{1}$.

- Where $\tau \geq 0$, some increasing function $u(\tau)$ is plurisubharmonic with respect to $J_{0}^{2}$.

- $\hat{\beta}_{+}^{2}=\widehat{\beta}_{-}^{2}$ for $\tau \geq 0$, where $\widehat{R_{ \pm}(\Gamma)}-\operatorname{int}\left(R_{ \pm}(\Gamma)\right)$ are naturally identified.

In particular, no holomorphic map from a punctured Riemann surface to the space $\left([0, \infty) \times \partial R_{ \pm}(\Gamma), J_{0}^{2}\right)$ has a local maximum of $\tau$ in the interior of the domain.

The 1 -form $\left(\alpha^{2}\right)^{*}$ on $M^{*}$ is defined as follows:

- $\left(\alpha^{2}\right)^{*}=\alpha^{1}$ on $M$.

- $\left(\alpha^{2}\right)^{*}=C d t+\widehat{\beta}_{ \pm}^{2}$ on $M^{*}-\operatorname{int}(M)$.

The almost complex structure $J^{2}$ on $\mathbb{R} \times M^{*}$ is chosen so that

- Conditions $\left(\mathrm{A}_{0}\right)$ (with respect to the 1 -form $\left.\left(\alpha^{2}\right)^{*}\right)$ and $\left(\mathrm{A}_{1}\right)$ from Section 3.1 hold;

- the projection of $J^{2}$ to $\widehat{R_{ \pm}(\Gamma)}$ is $J_{0}^{2}$;

- $J^{2}=J^{1}$ on $\mathbb{R} \times\{\tau \leq 0\}$.

We then apply Step 1 to obtain a chain map

$$
\Phi_{1}: \mathcal{A}\left(\alpha^{0}, J^{0}\right) \rightarrow \mathcal{A}\left(\left(\alpha^{2}\right)^{*}, J^{2}\right),
$$

which is a quasi-isomorphism.

On the other hand, since $J_{0}^{1}$ and $J_{0}^{2}$ agree on $R_{ \pm}(\Gamma)$ and $\tau \circ F$ does not attain a local maximum for any holomorphic curve $F$ where $\tau>0$, it follows that every holomorphic curve counted in $\partial$ for $J_{0}^{2}$ lies inside $\mathbb{R} \times\{\tau \leq 0\}$. This implies that $\mathcal{A}\left(\left(\alpha^{2}\right)^{*}, J^{2}\right)=\mathcal{A}\left(\alpha^{1}, J^{1}\right)$ as chain complexes. Hence we obtain an isomorphism

$$
H C\left(M, \Gamma, \alpha^{0}, J^{0}\right) \stackrel{\simeq}{\rightarrow} H C\left(M, \Gamma, \alpha^{1}, J^{1}\right) .
$$

Step 3 To complete the proof of the proposition, we need to show that the isomorphism (20) is canonical when $\xi^{\lambda}$ is independent of $\lambda$, and otherwise depends only on the homotopy class of the path $\left\{\xi^{\lambda}\right\}$.

First consider the situation where $M$ is closed and $\alpha_{0}, \alpha_{1}$ are contact $1-$ forms which are homotopic through contact 1 -forms $\alpha_{\rho}, \rho \in[0,1]$. We can use the homotopy to construct a cobordism $(\mathbb{R} \times M, J)$ giving rise to the chain map $\Phi: \mathcal{A}\left(\alpha_{0}, J_{0}\right) \rightarrow \mathcal{A}\left(\alpha_{1}, J_{1}\right)$, 
where $J_{i}$ is adapted to $\alpha_{i}$. Now, if there are two homotopies $\alpha_{\rho}, \alpha_{\rho}^{\prime}$ from $\alpha_{0}$ to $\alpha_{1}$ which are homotopic, then there is a homotopy of cobordisms from $(\mathbb{R} \times M, J)$ to $\left(\mathbb{R} \times M, J^{\prime}\right)$, and the usual chain homotopy argument implies that the induced isomorphisms $\Phi, \Phi^{\prime}$ agree. In other words, the map $\Phi: H C\left(M, \alpha_{0}, J_{0}\right) \rightarrow H C\left(M, \alpha_{1}, J_{1}\right)$ only depends on the homotopy class of paths connecting $\alpha_{0}$ and $\alpha_{1}$; however, the map will likely depend on the choice of homotopy class. On the other hand, when we have two contact 1 -forms $\alpha_{0}$ and $\alpha_{1}$ for the same contact structure $\xi$, we can write $\alpha_{1}=f_{1} \alpha_{0}$, and there is a canonical homotopy class of paths from $\alpha_{0}$ to $\alpha_{1}$, namely one which has the form $\alpha_{\rho}=f_{\rho} \alpha_{0}$. Hence, the identification $\Phi: H C\left(M, \alpha_{0}, J_{0}\right) \rightarrow$ $H C\left(M, f_{1} \alpha_{0}, J_{1}\right)$ is canonical.

Returning to the sutured case, suppose $\alpha^{0}$ and $\alpha^{1}$ are adapted to the sutured contact manifold $(M, \Gamma, U(\Gamma), \xi)$. We claim that the contact homology algebras $H C\left(M, \Gamma, \alpha^{0}, J^{0}\right)$ and $H C\left(M, \Gamma, \alpha^{1}, J^{1}\right)$ are canonically isomorphic. Since $\alpha^{0}$ and $\alpha^{1}$ are contact forms for the same contact structure $\xi$, the forms $\alpha^{1}$ and $\alpha^{0}$ are conformally equivalent. Consequently, $\left(\beta_{ \pm}^{1}\right)_{0}$ and $\left(\beta_{ \pm}^{0}\right)_{0}$ differ by a constant multiple. Any two almost complex structures $J$ constructed in the proof of Lemma 3.2 are connected by a 1 -parameter family of almost complex structures with the same properties. Hence there is a 1parameter family of chain maps $\left(\Phi_{1}\right)_{\rho}:\left(\alpha^{0}, J^{0}\right) \rightarrow \mathcal{A}\left(\left(\alpha^{2}\right)_{\rho}^{*}, J_{\rho}^{2}\right)$ where $\mathcal{A}\left(\left(\alpha^{2}\right)_{\rho}^{*}, J_{\rho}^{2}\right)$ and $\mathcal{A}\left(\alpha^{1}, J^{1}\right)$ are canonically isomorphic. Then, by the discussion in the previous paragraph, the induced isomorphisms in Equation (20) agree.

\subsection{Sutured embedded contact homology}

Suppose now that $(M, \Gamma, \alpha)$ is a sutured contact manifold where $\operatorname{dim}(M)=3$ and $\alpha$ is nondegenerate. Let $J$ be a generic tailored almost complex structure on $\mathbb{R} \times M^{*}$. We can now define the sutured embedded contact homology $\operatorname{ECH}(M, \Gamma, \alpha, J)$ by copying the definition in the closed case (see eg [28, Section 7]) verbatim. It follows from the discussion at the end of Section 6.1 that for generic tailored $J$, the moduli spaces of $J$-holomorphic curves needed to define the ECH differential $\partial$ and prove that $\partial^{2}=0$ are cut out transversely. (These curves are all somewhere injective.) Corollary 5.21 implies that the necessary compactness holds to show that $\partial$ is defined and satisfies $\partial^{2}=0$. The gluing analysis from $[28 ; 29]$ to complete the proof that $\partial^{2}=0$ carries over unchanged.

Recall that part of Conjecture 1.2 is that $\operatorname{ECH}(M, \Gamma, \alpha, J)$ depends only on $(M, \alpha, \xi)$. Currently the only known proof of the analogous statement in the closed case uses Seiberg-Witten theory; there is no known definition of an isomorphism in terms of holomorphic curves (due to the presence of multiply covered curves of negative ECH index in cobordisms). However if such an isomorphism could be constructed, then the discussion in Section 6.2 would allow it to be extended to the sutured case. 


\section{Variations}

In this section we define some variants of sutured contact homology and sutured ECH.

\subsection{The "hat" versions of contact homology and embedded contact ho- mology}

Let $(M, \xi)$ be a closed contact $(2 n+1)$-dimensional manifold. Choose a contact form $\alpha$ for $\xi$ and a Darboux ball $\left(B^{2 n+1}=D^{2 n} \times[-1,1],\left.\operatorname{ker} \alpha\right|_{B^{2 n+1}}\right) \subset(M, \xi)$ such that $D^{2 n} \times[-1,1]$ has coordinates $\left(x_{1}, y_{1}, \ldots, x_{n}, y_{n}, t\right), D^{2 n}=\left\{\sum_{i}\left|x_{i}\right|^{2}+\left|y_{i}\right|^{2} \leq 1\right\}$, and $\left.\alpha\right|_{B^{2 n+1}}=d t+\sum_{i} \frac{1}{2}\left(x_{i} d y_{i}-y_{i} d x_{i}\right)$. On $B^{2 n+1}$, the Reeb vector field is given by $R_{\alpha}=\partial_{t}$. In particular, $R_{\alpha}$ is tangent to $\left(\partial D^{2 n}\right) \times[-1,1]$ and transverse to $D^{2 n} \times\{-1,1\}$. Let $\left(M(1)^{\prime},\left.\alpha\right|_{M(1)^{\prime}}\right)$ be the concave sutured contact manifold obtained from $(M, \alpha)$ by removing $B^{2 n+1}$. Applying the concave-to-convex procedure described in Section 4.2 to $\left(M(1)^{\prime},\left.\alpha\right|_{M(1)^{\prime}}\right)$ then gives a convex sutured contact manifold $\left(M(1), \alpha_{1}\right)$.

Recall from Theorem 1.6 that when $\operatorname{dim}(M)=3$ we have

$$
\widehat{E C H}(M, \xi) \simeq E C H\left(M(1), \alpha_{1}\right) .
$$

By analogy with this, in all odd dimensions we define a "hat" version of contact homology by

$$
\widehat{H C}(M, \xi)=H C\left(M(1), \alpha_{1}\right) .
$$

(This does not depend on $\alpha$ as shown in Section 6.2.)

\subsection{A transverse knot filtration}

Let $(M, \xi)$ be a closed contact 3-manifold and let $K \subset M$ be a null-homologous transverse knot. Since $K$ is transverse, there exists a contact form $\alpha$ on $M$ such that $\xi=\operatorname{ker} \alpha$ and $K$ is a closed orbit of $R_{\alpha}$. In fact, by the Darboux-Weinstein neighborhood theorem, we can choose $\alpha$ so that there is a neighborhood $N(K)=$ $D^{2} \times[-2,2] /(-2 \sim 2)$ of $K=\{r=0\}$ on which $\alpha=d t+c r^{2} d \theta$. Here $c$ is a small positive constant, $(r, \theta, t)$ are cylindrical coordinates on $D^{2} \times[-2,2]$, and $D^{2}=\{r \leq 1\}$. Let $\left(M(1)^{\prime},\left.\alpha\right|_{M(1)^{\prime}}\right)$ be defined as in the previous section, where $B^{3}=D^{2} \times[-1,1] \subset N(K)$. Define $\left(M(1), \alpha_{1}\right)$ as above, so that (21) and (22) hold.

Next we define a related contact manifold $\left(M_{0}, \Gamma_{0}, \xi_{0}\right)$, which is obtained from $\left(M-N(K),\left.\xi\right|_{M-N(K)}\right)$ by attaching a collar. Consider

$$
A=\partial(M-N(K)) \times[-1,0]=\mathbb{R} / 2 \pi \mathbb{Z} \times([-2,2] / \sim) \times[-1,0]
$$


with coordinates $(\theta, t, u)$. We take $M_{0}=(M-N(K)) \cup A$, where $\partial(M-N(K))$ is identified with $\partial(M-N(K)) \times\{-1\}$. We extend $\alpha$ over $A$ as $d t-c u d \theta$. (This is smooth if we define the smooth structure on $M_{0}$ using an appropriate chart in the gluing region.) If we perturb $\alpha$ near $\partial(M-N(K)) \times\{0\}$, then the resulting $\xi_{0}=\operatorname{ker} \alpha_{0}$ has convex boundary and dividing set $\Gamma_{0}$ which consists of two meridians (circles where $t$ is constant).

Proposition 7.1 A nullhomologous transverse knot $K$ in a closed contact 3-manifold $(M, \xi)$ induces

(1) a filtration $\mathcal{F}$ on the chain complex $C\left(M(1), \alpha_{1}\right)$ for $\widehat{H C}(M, \xi)$, such that the homology of the associated graded complex is isomorphic to $\mathrm{HC}\left(M_{0}, \Gamma_{0}, \xi_{0}\right)$;

(2) a filtration $\mathcal{F}$ on the chain complex for $\operatorname{ECH}\left(M(1), \alpha_{1}, J_{1}\right) \simeq \widehat{E C H}(M, \xi)$, such that the homology of the associated graded complex is isomorphic to $\operatorname{ECH}\left(M_{0}, \Gamma_{0}, \alpha_{0}, J_{0}\right)$, if the almost complex structures $J_{0}$ and $J_{1}$ are suitably related.

Proof We will only prove assertion (1) for sutured contact homology; assertion (2) for sutured $\mathrm{ECH}$ is proved using the same argument.

A generator of $C\left(M(1), \alpha_{1}\right)$ is a monomial $\underline{\gamma}=\gamma_{1}^{m_{1}} \cdots \gamma_{k}^{m_{k}}$, where the $\gamma_{i}$ are closed orbits of $R_{\alpha_{1}}$, and each $m_{i}$ is a positive integer. The total homology class of this generator is $A=m_{1}\left[\gamma_{1}\right]+\cdots+m_{k}\left[\gamma_{k}\right] \in H_{1}(M)$. Fix a relative homology class $B \in H_{2}(M, K)$ with $\partial B=[K]$, and let $S$ be a Seifert surface for $K$ in the class $B$. Let $K_{1}=K \cap M(1)$. We view $S$ as a surface in $M(1)$ with boundary on $K_{1} \cup \partial M(1)$. Since all the closed orbits of $R_{\alpha_{1}}$ are contained in $M(1) \backslash K_{1}$, we can define the filtration level of $\underline{\gamma}$ to be its algebraic intersection number with $S$, namely

$$
\mathcal{F}(\underline{\gamma})=\underline{\gamma} \cdot S=\sum_{i=1}^{k} m_{i}\left(\gamma_{i} \cdot S\right) .
$$

Note that if $\underline{\gamma}^{\prime}=\left(\gamma_{1}^{\prime}\right)^{m_{1}^{\prime}} \cdots\left(\gamma_{l}^{\prime}\right)^{m_{l}^{\prime}}$ is another generator representing the same homology class $A \in \overline{H_{1}}(M)$, then the filtration difference is given by

$$
\mathcal{F}(\underline{\gamma})-\mathcal{F}\left(\underline{\gamma^{\prime}}\right)=\Sigma \cdot K_{1}
$$

where $\Sigma$ is any $2-$ chain in $M$ with $\partial \Sigma=\sum_{i=1}^{k} m_{i} \gamma_{i}-\sum_{j=1}^{l} m_{j}^{\prime} \gamma_{j}^{\prime}$. One can show this by perturbing $\Sigma$ so that it is transverse to $S$ and then counting points in the boundary of the compact 1 -manifold $\Sigma \cap S$. 
Next we prove that the differential does not increase the filtration level of the generators. More generally, for any holomorphic curve

$$
F=(a, f):(\Sigma, j, \mathbf{m}) \rightarrow \mathbb{R} \times M(1)^{*}
$$

which is positively asymptotic to $\underline{\gamma}$ and negatively asymptotic to $\underline{\gamma^{\prime}}$, we have

$$
\mathcal{F}(\underline{\gamma}) \geq \mathcal{F}\left(\underline{\gamma}^{\prime}\right) \text {. }
$$

To prove this, first note that $K_{1}$ extends to an infinite length Reeb orbit $\widetilde{K}_{1}$ in $M(1)^{*}$. Now let $\bar{\Sigma}$ be the compact surface with boundary obtained from $\Sigma$ by performing a real blowup at each puncture. Then the map $f$ extends to a map $\bar{f}: \bar{\Sigma} \rightarrow M(1)^{*}$ whose restriction to the boundary is $\sum_{i} m_{i} \gamma_{i}-\sum_{j} m_{j}^{\prime} \gamma_{j}^{\prime}$. Moreover $f$ is homotopic rel boundary to a map $f^{\prime}$ whose image is contained in $M(1)$. We then have

$$
\mathcal{F}(\underline{\gamma})-\mathcal{F}\left(\underline{\gamma}^{\prime}\right)=f^{\prime}(\bar{\Sigma}) \cdot K_{1}=f(\bar{\Sigma}) \cdot \tilde{K}_{1} \geq 0,
$$

where the last inequality holds by positivity of intersections of the holomorphic curve $F$ with the holomorphic plane $\mathbb{R} \times \widetilde{K}_{1}$ in $\mathbb{R} \times M(1)^{*}$.

We now show that the homology of the associated graded complex with respect to $\mathcal{F}$ is the contact homology $H C\left(M_{0}, \Gamma_{0}, \xi_{0}\right)$. Recall the identification of $N(K)$ with the set $D^{2} \times[-2,2] /(-2 \sim 2)$. Consider a small neighborhood $N\left(K_{1}\right)=$ $D_{\varepsilon}^{2} \times([-2,-1] \cup[1,2]) \subset M(1) \cap N(K)$, where $D_{\varepsilon}^{2}=\{r \leq \varepsilon\}$. The manifold $M(1)-N\left(K_{1}\right)$ is almost a convex sutured manifold contactomorphic to $\left(M_{0}, \Gamma_{0}, \xi_{0}\right)$. The only issue is that, along $\left(\partial D_{\varepsilon}^{2}\right) \times\{t\}$ with $t \in[-2,1] \cup[1,2]$, the contact form $\alpha$ restricts to a positive contact form with respect to the boundary orientation induced from $D_{\varepsilon}^{2}$, and hence to a negative contact form with respect to the boundary of $R_{ \pm}(\Gamma)$. To remedy this problem we attach a collar

$$
A^{\prime}=\mathbb{R} / 2 \pi \mathbb{Z} \times([-2,-1] \cup[1,2]) \times[-1,1]
$$

with coordinates $(\theta, t, u)$ to $M(1)-N\left(K_{1}\right)$ by identifying $\partial D_{\varepsilon}^{2} \times([-2,-1] \cup[1,2])$ with $\mathbb{R} / 2 \pi \mathbb{Z} \times([-2,-1] \cup[1,2]) \times\{-1\}$ and extending via the contact form $d t-c u d \theta$. Then $\left(M(1)-N\left(K_{1}\right)\right) \cup A^{\prime}$ is a sutured contact manifold, and we leave it as an exercise to prove that it is contactomorphic to $\left(M_{0}, \Gamma_{0}, \xi_{0}\right)$ (modulo the process of matching up the contact structures on the boundary by a homotopy).

Finally, let $N\left(\widetilde{K}_{1}\right)$ denote the obvious extension of $N\left(K_{1}\right)$ to a neighborhood of $\widetilde{K}_{1}$ in $M(1)^{*}$. We then observe that a holomorphic curve in $\mathbb{R} \times M(1)^{*}$ does not pass through $\mathbb{R} \times \widetilde{K}_{1}$, ie does not decrease the filtration, if and only if its image is contained in $\mathbb{R} \times\left(M(1)^{*}-N\left(\widetilde{K}_{1}\right)\right)$. This follows from intersection positivity by observing that $N\left(\widetilde{K}_{1}\right)$ is foliated by Reeb arcs parallel to $\widetilde{K}_{1}$. A similar argument shows that the holomorphic curves that are counted by the contact homology differential 
in $\mathbb{R} \times\left(\left(\left(M(1)-N\left(K_{1}\right)\right) \cup A^{\prime}\right)^{*}\right.$ do not pass through the "vertical completion" of $\mathbb{R} \times A^{\prime}$, and so are contained in $\mathbb{R} \times\left(M(1)^{*}-N\left(\widetilde{K}_{1}\right)\right)$. Thus the differential on the associated graded complex for $M(1)$ counts the same holomorphic curves as the differential for the contact homology of $\left(M(1)-N\left(K_{1}\right)\right) \cup A^{\prime} \simeq M_{0}$.

Remark 7.2 Although the filtration defined above depends on the choice of a relative homology class $B \in H_{2}(M, K)$ with $\partial B=[K]$, the filtration difference between two generators representing the same class $A \in H_{1}(M)$ does not depend on this choice, by Equation (23).

\subsection{Invariants of Legendrian submanifolds}

In this section we briefly discuss invariants of Legendrian submanifolds. Let $(M, \xi)$ be a closed $(2 n+1)$-dimensional contact manifold and $L \subset M$ be a closed Legendrian submanifold. By Example 4.5, there is a tubular neighborhood $N(L)$ of $L$ so that $\left(M-N(L), \Gamma=S^{n-1} T^{*} L,\left.\xi\right|_{M-N(L)}\right)$ is a concave sutured contact manifold. Now, by Proposition 4.6, we can modify the concave sutured contact manifold into a convex sutured contact manifold $\left(M^{\prime}, \Gamma^{\prime}, \xi^{\prime}\right)$. Then we define

$$
H C(M, \xi, L)=H C\left(M^{\prime}, \Gamma^{\prime}, \xi^{\prime}\right)
$$

To show that this is well-defined, recall from Section 6.2 that the right hand side is independent of the choices of contact form and the almost complex structure. We then have:

Lemma 7.3 The contact homology algebra $H C(M, \xi, L)$ is an invariant of $(M, \xi, L)$, ie does not depend on the choice of tubular neighborhood of $L$.

Proof Observe that the hypersurface $\Sigma$ of $M$, defined in Example 4.5, has the following properties:

(i) There is a contact 1 -form for $\xi$, written locally as

$$
\alpha=d z+\beta=d z+\sum_{i=1}^{n} f_{i}(p, q) d p_{i}+\sum_{i=1}^{n} g_{i}(p, q) d q_{i} .
$$

Here $\left(z, p=\left(p_{1}, \ldots, p_{n}\right), q=\left(q_{1}, \ldots, q_{n}\right)\right)$ are local coordinates, $R_{\alpha}=\partial_{z}$, $L=\{z=0, p=0\}$, and $f_{i}(0, q)=g_{i}(0, q)=0$ for all $q$. In particular, $\xi$ is tangent to $\{z=0\}$ along $L$. 
(ii) On the $2 n$-dimensional submanifold $\{z=0\}$, let $Y$ be the Liouville vector field satisfying $l_{Y} d \beta=\beta$, and let $W_{q}$ be the "fan" consisting of all points $(p, q)$ whose backwards flow along $Y$ converge to $(0, q)$. Then let $\Gamma$ be a $(2 n-1)$-dimensional submanifold of $\{z=0\}$ which is arbitrarily close to $L$ and such that each $\Gamma \cap W_{q}$ is "star-shaped", ie an (n-1)-dimensional sphere which is transverse to $Y$.

(iii) The submanifold $\Gamma$ is diffeomorphic to the unit cotangent bundle of $L$ and bounds a $2 n$-dimensional submanifold $\Sigma_{0} \subset\{z=0\}$ which is diffeomorphic to the unit disk bundle of $T^{*} L$. Then $\Sigma \cap\{z>0\}$ (resp. $\Sigma \cap\{z<0\}$ ) is transverse to $R_{\alpha}$ and the projection along $R_{\alpha}$ gives a diffeomorphism with $\operatorname{int}\left(\Sigma_{0}\right)$.

Condition (ii) implies that $\Gamma$ is a $(2 n-1)$-dimensional contact submanifold and Condition (iii) implies that $\Sigma$ is a convex hypersurface of $M$.

Now let $\alpha$ be a contact 1 -form for $\xi$, which is defined in a neighborhood of $L$ and satisfies (i). In particular, $\alpha$ is given by Equation (24). We describe the Liouville vector field $Y$ for $\beta$ on $\{z=0\}$ when $|p|$ is arbitrarily small. For $|p|$ small,

$$
d \alpha \approx \sum_{i} \partial g_{i} \partial p_{j} d p_{j} d q_{i}+\sum_{i} \partial f_{i} \partial p_{j} d p_{j} d p_{i}
$$

since $\partial f_{i} / \partial q_{j}$ and $\partial g_{i} / \partial q_{j}$ are close to zero. Ignoring higher order terms, we write $f_{i}=\sum_{j} F_{i j} p_{j}$ and $g_{i}=\sum_{j} G_{i j} p_{j}$, where $F_{i j}$ and $G_{i j}$ are constants. By the symplectic condition, $\operatorname{det}\left(\partial g_{i} / \partial p_{j}\right)=\operatorname{det}\left(G_{i j}\right)>0$. If we write $Y=\sum_{i} a_{i} \partial_{p_{i}}+\sum_{i} b_{i} \partial_{q_{i}}$, then the Liouville condition implies that

$$
g_{i}=\sum_{j} \partial g_{i} \partial p_{j} a_{j}
$$

or $\sum_{j} G_{i j} p_{j}=\sum_{j} G_{i j} a_{j}$. Hence $a_{j}=p_{j}$ and $Y$ has the form

$$
Y=\sum_{i} p_{i} \partial_{p_{i}}+\sum_{i, j} A_{i j} p_{j} \partial_{q_{j}},
$$

by the invertibility of $G_{i j}$. Here $A_{i j}$ are constants which smoothly depend on $F_{i j}$ and $G_{i j}$.

Equation (25) implies that the fan $W_{q}$ and $\left.Y\right|_{W_{q}}$ vary continuously as we vary $\beta$ (while preserving the conditions in (i)), and that the $\partial_{p_{i}}$-terms are independent of $\beta$ (modulo higher order corrections).

Finally, given two convex submanifolds $\Sigma^{0}$ and $\Sigma^{1}$ of the type described in Example 4.5, there is a 1-parameter family of contact 1 -forms $\alpha^{t}$ interpolating between $\alpha^{0}$ and $\alpha^{1}$, all satisfying (i). Since $W_{q}^{t}$ varies continuously with $\beta^{t}$, it follows that there is a 
family $\Gamma^{t}$ from $\Gamma^{0}$ to $\Gamma^{1}$, all satisfying (ii). We can then extend to $\Sigma^{t}$ from $\Sigma^{0}$ to $\Sigma^{1}$, all satisfying (iii). This implies that $\Sigma^{0}$ and $\Sigma^{1}$ can be connected by a 1 -parameter family of convex submanifolds $\Sigma^{t}$. To conclude, Lemma 4.1 provides a 1 -parameter family of sutured contact manifolds $\left\{\left(M^{\prime}, \Gamma^{\prime}, \xi_{t}^{\prime}\right)\right\}_{t \in[0,1]}$, whose contact homology groups are isomorphic by Proposition 6.1(2).

Our Legendrian submanifold invariant $H C(M, \xi, L)$, unlike other invariants such as Legendrian contact homology, does not automatically vanish under stabilizations. In fact, Corollary 1.12 shows that the invariant does not vanish for example when the ambient manifold $(M, \xi)$ has an exact symplectic filling.

Example 7.4 Suppose $(M, \xi)=\left(S^{3}, \xi\right)$ is the standard contact 3-sphere and $L$ is a Legendrian unknot with Thurston-Bennequin number $t b(L)=-n$ and rotation number $r(L)=n-1$ for $n \geq 1$. (These Legendrian unknots have maximal rotation number amongst those with the same $t b$.) Then $\left(S^{3}-N(L),\left.\xi\right|_{S^{3}-N(L)}\right)$ is a sutured contact solid torus which is obtained from a product sutured contact manifold

$$
\left(D^{2} \times[-1,1], \partial D^{2} \times\{0\}, N\left(\partial D^{2}\right) \times[-1,1], d t+\beta\right),
$$

where $\beta$ is a primitive of an area form on $D^{2}$, by a sutured manifold gluing. Its contact homology $H C\left(S^{3}, \xi, L\right)$ has been completely calculated by Golovko [18; 17], and in particular is nonzero.

Question 7.5 Determine the relationship of $H C(M, \xi, L)$ with the Legendrian contact homology $L C H(M, \xi, L)$ of the Legendrian submanifold $L \subset(M, \xi)$ as well as the contact homology $H C\left(M(L), \xi_{L}\right)$ of the contact manifold $\left(M(L), \xi_{L}\right)$, obtained from $M$ by Legendrian surgery along $L$. (A surgery exact sequence involving $H C\left(M(L), \xi_{L}\right)$ and a variant of $\operatorname{LCH}(M, \xi, L)$ was obtained by Bourgeois, Ekholm and Eliashberg [2].)

When $\operatorname{dim} M=3$, we can also define

$$
\operatorname{ECH}(M, \xi, L)=\operatorname{ECH}\left(M^{\prime}, \Gamma^{\prime}, \xi^{\prime}\right) .
$$

This is conjectured to be independent of the choice of $\xi$ (up to the usual grading shift) and dependent only on the framing of $L$.

\section{First warm-up: neck-stretching in the $t$-direction}

Before embarking on the proof of Theorem 1.9, we treat slightly easier cases in this section and the next. 
Consider the situation where we have a sutured contact manifold $\left(M^{\prime}, \Gamma^{\prime}, \alpha^{\prime}\right)$, and there is a diffeomorphism

$$
\phi:\left(R_{+}\left(\Gamma^{\prime}\right), \beta_{+}^{\prime}\right) \stackrel{\sim}{\rightarrow}\left(R_{-}\left(\Gamma^{\prime}\right), \beta_{-}^{\prime}\right),
$$

where $\beta_{ \pm}^{\prime}=\left.\alpha^{\prime}\right|_{R_{ \pm}\left(\Gamma^{\prime}\right)}$, which is the identity on $R_{+}\left(\Gamma^{\prime}\right) \cap U\left(\Gamma^{\prime}\right)$. Let $(M, \alpha)$ be the contact manifold with boundary obtained from $M^{\prime}$ by gluing $R_{+}\left(\Gamma^{\prime}\right)$ and $R_{-}\left(\Gamma^{\prime}\right)$ via $\phi$. If we let $\Gamma$ denote the image of $\Gamma^{\prime}$ in $M$, then a neighborhood of $\partial M$ is identified with $[-1,0] \times(\mathbb{R} / \mathbb{Z}) \times \Gamma$ so that $\alpha=C d t+\beta$.

Although $(M, \alpha)$ is not quite a sutured contact manifold in the sense of Definition 2.8, we can nonetheless define part of its contact homology as follows. First complete $(M, \alpha)$ to $\left(M^{*}, \alpha^{*}\right)$ by attaching the side (S) as usual (but not the top/bottom), and choose a tailored almost complex structure on $\mathbb{R} \times M^{*}$. Let $\mathcal{A}_{[0]}(M, \Gamma, \alpha)$ be the free supercommutative $\mathbb{Q}$-algebra with unit generated by good Reeb orbits in $M^{*}$ which do not intersect $R_{+}\left(\Gamma^{\prime}\right)$; note that these are the same as the good Reeb orbits in $M^{\prime}$. Note that if a holomorphic curve in $\mathbb{R} \times M^{*}$ has all positive ends at such Reeb orbits, then it also has all negative ends at such Reeb orbits; otherwise the sum of the Reeb orbits from the positive ends could not be homologous to the sum of the Reeb orbits from the negative ends, because all orbits that nontrivially intersect $R_{+}\left(\Gamma^{\prime}\right)$ do so positively. Thus the usual construction defines a well-defined differential on $\mathcal{A}_{[0]}(M, \Gamma, \alpha)$ which has a well-defined homology $H C_{[0]}(M, \Gamma, \alpha)$.

The goal of this section is to prove the following result:

Theorem 8.1 There is an isomorphism $H C\left(M^{\prime}, \Gamma^{\prime}\right) \simeq H C_{[0]}(M, \Gamma)$.

The idea of the proof is to "stretch the neck" in the gluing that produces $M$ from $M^{\prime}$, with a parameter $n$ that measures the length of the neck. One wants to argue that if $n$ is sufficiently large then all relevant holomorphic curves in $\mathbb{R} \times M^{*}$ correspond to holomorphic curves in $\mathbb{R} \times\left(M^{\prime}\right)^{*}$. However, one cannot choose a single $n$ that always works; the size of $n$ that is required for this to work depends on the total symplectic action of the Reeb orbits involved. To deal with this issue we will use a direct limit argument.

We remark that one can also prove a more general version of Theorem 8.1 in which one glues only some components of $R_{+}\left(\Gamma^{\prime}\right)$ to some components of $R_{-}\left(\Gamma^{\prime}\right)$. This uses the same argument but more notation.

\subsection{Stretching the neck}

For the purposes of the neck-stretching, we introduce a sequence of contact manifolds with boundary $\left(M_{n}, \alpha_{n}\right)$ and almost complex structures $J_{n}$ which are parametrized 
by $n$ : Let $M_{n}$ be the manifold diffeomorphic to $M=M^{\prime} / \phi$, obtained from the disjoint union $M^{\prime} \sqcup\left(R_{+}\left(\Gamma^{\prime}\right) \times[-n, n]\right)$ by identifying $R_{+}\left(\Gamma^{\prime}\right)$ with $R_{+}\left(\Gamma^{\prime}\right) \times\{-n\}$ by the identity and $R_{+}\left(\Gamma^{\prime}\right) \times\{n\}$ with $R_{-}\left(\Gamma^{\prime}\right)$ by $\phi$. We take the $1-$ form $\alpha_{n}$ to agree with $d t+\beta_{+}^{\prime}$ on $R_{+}\left(\Gamma^{\prime}\right) \times[-n, n]$ and with $\alpha^{\prime}$ on $M^{\prime}$. Let $J^{\prime}$ be an almost complex structure which is tailored to $\left(M^{\prime}, \alpha^{\prime}\right)$ and is taken to itself by $\phi$. Then define $J_{n}$ to be $t$-invariant on $R_{+}\left(\Gamma^{\prime}\right) \times[-n, n]$ and to agree with $J^{\prime}$ on $M^{\prime}$. Also define $M_{n}^{*}$ as the completion of $M_{n}$, obtained by attaching (S), but not (T) or (B) since $R_{ \pm}$have been eliminated. By counting $J_{n}$-holomorphic curves in $\mathbb{R} \times M_{n}^{*}$ we can define the contact homology $H C_{[0]}\left(M_{n}, \alpha_{n}, J_{n}\right)$. The standard continuation argument shows that this does not depend on $n$ and is canonically isomorphic to $H C_{[0]}(M, \Gamma)$.

Lemma 8.2 Let $\underline{\gamma}^{+}=\left(\gamma_{1}^{+}, \ldots, \gamma_{k}^{+}\right)$and $\underline{\gamma}^{-}=\left(\gamma_{1}^{-}, \ldots, \gamma_{l}^{-}\right)$be finite ordered sets of Reeb orbits in $M^{\prime}$, possibly taken with multiplicities. Then given $g$, for all sufficiently large $n$,

$$
\mathcal{M}_{g}\left(\underline{\gamma}^{+} ; \underline{\gamma}^{-} ; \mathbb{R} \times\left(M^{\prime}\right)^{*}, J^{\prime}\right)=\mathcal{M}_{g}\left(\underline{\gamma}^{+} ; \underline{\gamma}^{-} ; \mathbb{R} \times M_{n}^{*}, J_{n}\right)
$$

Proof The proof is almost identical to that of Lemma 5.17 and Proposition 5.18; the slight difference is that the ranges of the holomorphic maps vary with $n$. Arguing by contradiction, suppose there is a sequence

$$
F_{n}=\left(a_{n}, f_{n}\right):\left(\Sigma_{n}, j_{n}, \mathbf{m}_{n}\right) \rightarrow\left(\mathbb{R} \times M_{n}^{*}, J_{n}\right)
$$

in $\mathcal{M}_{g}\left(\gamma^{+} ; \gamma^{-} ; \mathbb{R} \times M_{n}^{*}, J_{n}\right)$ whose second component $f_{n}$ nontrivially intersects $\widehat{R_{+}\left(\Gamma^{\prime}\right)} \times\{0\}$ for all $n$. (Observe that, if $f_{n}$ does not intersect $\widehat{R_{+}\left(\Gamma^{\prime}\right)} \times\{0\}$, then $F_{n}$ can be viewed as a holomorphic map in $\mathcal{M}_{g}\left(\gamma^{+} ; \underline{\gamma}^{-} ; \mathbb{R} \times\left(M^{\prime}\right)^{*}, J^{\prime}\right)$.) As before, we can restrict to $\tau \leq 0$ by the strict plurisubharmonicity of $\tau$.

On $\mathbb{R} \times M_{n}^{*}$ we use the metric given by Equation (15), and on $\left(\Sigma_{n}-\mathbf{m}_{n}, j_{n}\right)$ we use the unique complete, compatible, finite volume hyperbolic metric $g_{n}$. Also write $\rho_{n}$ for the injectivity radius of $g_{n}$. If there is no "gradient bound", ie a bound on $\rho_{n}(x)\left\|\nabla F_{n}(x)\right\|$, then we obtain the bubbling off of a nonconstant finite energy plane with image in $\left(\mathbb{R} \times\left(M^{\prime}\right)^{*}, J^{\prime}\right)$ or $\left(\mathbb{R} \times \mathbb{R} \times \widehat{R_{+}\left(\Gamma^{\prime}\right)}, J^{\prime}\right)$ by Lemma 5.9 . In the latter case, we obtain a holomorphic sphere inside $\left(\mathbb{R} \times \mathbb{R} \times \widehat{R_{+}\left(\Gamma^{\prime}\right)}, J^{\prime}\right)$ by the removal of singularities lemma for the Top/Bottom (Corollary 5.15), a contradiction. Hence the bubbling occurs inside $\left(\mathbb{R} \times\left(M^{\prime}\right)^{*}, J^{\prime}\right)$. Since the area of finite energy holomorphic planes is bounded by below (see [3, Lemma 5.11]), we can remove finite sets $\mathbf{m}_{n}^{0}$ from $\Sigma_{n}-\mathbf{m}_{n}$ to ensure that there is a gradient bound with respect to $\left(\dot{\Sigma}_{n}=\Sigma_{n}-\left(\mathbf{m}_{n} \cup \mathbf{m}_{n}^{0}\right), j_{n}\right)$. 
Arguing as in Proposition 5.18, there is a subsequence of $F_{n}$ (again denoted $F_{n}$ by abuse of notation) for which

(i) there is a bound on the gradient;

(ii) there is a $\varepsilon$-thin component $C^{n}$ of $\dot{\Sigma}_{n}$ and an annulus $Z^{n} \subset C^{n}$, such that $f_{n}\left(Z^{n}\right) \subset R_{+}\left(\Gamma^{\prime}\right) \times[-n, n]$

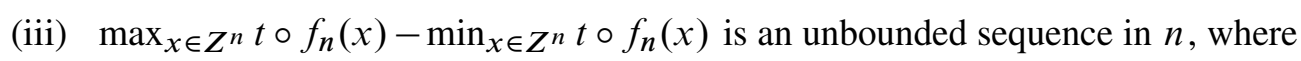
$t \in[-n, n]$.

This sequence limits to a nonconstant holomorphic cylinder in $\left(\mathbb{R} \times \mathbb{R} \times \widehat{R_{+}\left(\Gamma^{\prime}\right)}, J^{\prime}\right)$, which is a contradiction.

\subsection{Continuation maps}

Given a contact form $\alpha$, the action of an oriented curve $\gamma$ with respect to $\alpha$ will be written as

$$
A_{\alpha}(\gamma)=\int_{\gamma} \alpha
$$

We also write $\bar{\gamma}=\gamma_{1}^{m_{1}} \cdots \gamma_{k}^{m_{k}}$ and $A_{\alpha}(\bar{\gamma})=\sum_{i} m_{i} A_{\alpha}\left(\gamma_{i}\right)$.

Let $\mathcal{A}_{\leq K}\left(M^{\prime}, \alpha^{\prime}, J^{\prime}\right)$ denote the subcomplex of $\mathcal{A}\left(M^{\prime}, \alpha^{\prime}, J^{\prime}\right)$ generated (as a module) by monomials $\bar{\gamma}$ with $A_{\alpha^{\prime}}(\bar{\gamma}) \leq K$. Lemma 8.2 implies that given $K$, if $n$ is sufficiently large then the inclusion

$$
\Phi_{K, n}: \mathcal{A}_{\leq K}\left(M^{\prime}, \alpha^{\prime}, J^{\prime}\right) \hookrightarrow \mathcal{A}\left(M_{n}, \alpha_{n}, J_{n}\right)
$$

is a chain map.

We now investigate the dependence of this map on $K$ and $n$. To start, we have the following key lemma:

Lemma 8.3 For all $n$ sufficiently large, the canonical isomorphism

$$
H C_{[0]}\left(M_{n}, \alpha_{n}, J_{n}\right) \simeq H C_{[0]}\left(M_{n+1}, \alpha_{n+1}, J_{n+1}\right)
$$

is induced by a chain map

$$
\Psi_{n}: \mathcal{A}_{[0]}\left(M_{n}, \alpha_{n}, J_{n}\right) \rightarrow \mathcal{A}_{[0]}\left(M_{n+1}, \alpha_{n+1}, J_{n+1}\right),
$$

such that if $\gamma$ is a Reeb orbit in $M^{\prime}$ then

$$
\gamma \mapsto \gamma+\sum_{i} a_{i} \overline{\gamma_{i}}
$$

where all the orbits of $\overline{\gamma_{i}}$ are contained in $M^{\prime}$ and $A_{\alpha_{n}}(\gamma)>A_{\alpha_{n+1}}\left(\overline{\gamma_{i}}\right)=A_{\alpha_{n}}\left(\overline{\gamma_{i}}\right)$. 
In particular, the lemma implies that the chain map $\Psi_{n}$ is "triangular", ie is the identity plus lower order terms with respect to the action.

Proof Let us write $\alpha^{1}=\alpha_{n}$; on $R_{+}\left(\Gamma^{\prime}\right) \times[-n, n], \alpha^{1}=d t+\beta$. (In this section we will write $\beta$ for $\beta_{+}^{\prime}$.) There exists an identification $i_{n}: M_{n} \stackrel{\sim}{\rightarrow} M_{n+1}$ so that $M^{\prime}$ is taken to itself by the identity and $i_{n}^{*}\left(\alpha_{n+1}\right)=f(t) d t+\beta$ on $R_{+}\left(\Gamma^{\prime}\right) \times[-n, n]$, where $1 \leq f(t) \leq 1+2 /(n-1)$. If we set $\alpha^{0}=i_{n}^{*}\left(\alpha_{n+1}\right)$, then $\alpha^{0}$ and $\alpha^{1}$ agree on $M^{\prime}$. Let $\alpha^{s}, s \in[0,1]$, be the 1 -parameter family of contact 1 -forms obtained by linearly interpolating between $\alpha^{0}$ and $\alpha^{1}$, perturbed to be $s$-independent near $s=0,1$. (These are contact forms if $n$ is sufficiently large.) Extend this family to all $s \in \mathbb{R}$ by setting $\alpha^{s}=\alpha^{0}$ for $s \leq 0$ and $\alpha^{s}=\alpha^{1}$ for $s \geq 1$.

Define an almost complex structure $J$ on $\mathbb{R} \times M_{n}^{*}$ such that the following hold:

(1) For all $s \in \mathbb{R},\left.J\right|_{\{s\} \times M_{n}^{*}}$ takes $\operatorname{ker} \alpha^{s}$ to itself, maps $\partial_{s}$ to $R_{\alpha^{s}}$, and is $d \alpha^{s}-$ positive.

(2) $\left.J\right|_{s \geq 1}=J_{n}$ and $\left.J\right|_{s \leq 0}=\left(\operatorname{id}_{\mathbb{R}} \times i_{n}\right)^{*} J_{n+1}$.

(3) $J$ is $s$-invariant on $\mathbb{R} \times M^{\prime}$.

(4) The projection of $\left.J\right|_{\mathbb{R} \times R_{+}\left(\Gamma^{\prime}\right) \times[-n, n]}$ to $\{s\} \times R_{+}\left(\Gamma^{\prime}\right) \times\{t\}$ does not depend on $s$ and on $t$.

The cobordism $\left(\mathbb{R} \times M_{n}^{*}, J\right)$ gives rise to the chain map $\Psi_{n}$, obtained in the usual way by counting rigid rational curves with one positive puncture and an unspecified number of negative punctures. The 2 -form $\omega$ that we use below to control the action is insufficient for verifying the compactness of the relevant moduli spaces. For compactness, we need a taming form $d\left(g(s) \alpha^{S}\right)$ for a suitable $g(s)$, whose $J-$ positivity is verified as in Lemma 3.2. We also restrict the range from $\mathbb{R} \times M_{n}^{*}$ to $\mathbb{R} \times M_{n}$; this is possible since the projection of $J$ to $\widehat{R_{+}\left(\Gamma^{\prime}\right)}$ is adapted to $\beta$.

Consider the 2-form $\omega=d \alpha^{1}$. We claim that $\omega$ is $J$-nonnegative, ie $\omega(v, J v) \geq 0$ for all tangent vectors $v$. On $M^{\prime}, \alpha^{1}=\alpha^{0}$, and the claim is immediate. On $R_{+}\left(\Gamma^{\prime}\right) \times[-n, n]$, we have $\omega=d \beta$. If we write $v \in T_{(s, x)}\left(\mathbb{R} \times R_{+}\left(\Gamma^{\prime}\right) \times[-n, n]\right)$ (for $s \in \mathbb{R}$ and $x \in R_{+}\left(\Gamma^{\prime}\right) \times[-n, n]$ ) as $a \partial_{s}+b \partial_{t}+w$, where $w \in \operatorname{ker} \alpha^{s}$, then $J v=a h \partial_{t}-(b / h) \partial_{s}+J_{s, t} w$. Here $h$ is a function which is approximately equal to 1 . (This comes from the fact that $R_{\alpha^{s}}$ is parallel, but not exactly equal, to $\partial_{t}$ on $\mathbb{R} \times R_{+}\left(\Gamma^{\prime}\right) \times[-n, n]$.) We then compute that

$$
\omega(v, J v)=d \beta\left(w, J_{s, t}(w)\right) \geq 0,
$$

by projecting to $R_{+}\left(\Gamma^{\prime}\right)$. 
Next let $F$ be a holomorphic curve in $\left(\mathbb{R} \times M_{n}, J\right)$ with positive end $\gamma$ and negative ends $\bar{\gamma}^{\prime}$. As noted previously, if $\gamma \subset M^{\prime}$, then all orbits of $\bar{\gamma}^{\prime}$ are also contained in $M^{\prime}$ for homological reasons. By Stokes' theorem and the $J$-nonnegativity of $\omega$, we have

$$
A_{\alpha^{1}}(\gamma) \geq A_{\alpha^{0}}\left(\bar{\gamma}^{\prime}\right)=A_{\alpha^{1}}\left(\bar{\gamma}^{\prime}\right) .
$$

Note that the first term on the right-hand side of Equation (26) comes from counting a trivial cylinder over $\gamma$. To obtain strict inequality in (27) when $\bar{\gamma}^{\prime} \neq \gamma$, first observe that $F$ is asymptotically a cylinder over $\gamma$ at $+\infty$. If $F$ is not a cylinder over $\gamma$, then $F$ must have positive $d \alpha^{1}$-area, implying the strict inequality in (27). (Branched covers of trivial cylinders do not contribute to the differential by Fabert [13].)

Our next ingredient is the following:

Lemma 8.4 Given $K>0$, there exists $n_{0}>0$ such that for all $n \geq n_{0}$,

$$
\Psi_{n}: \mathcal{A}_{[0]}\left(M_{n}, \alpha_{n}, J_{n}\right) \rightarrow \mathcal{A}_{[0]}\left(M_{n+1}, \alpha_{n+1}, J_{n+1}\right)
$$

maps $\gamma \mapsto \gamma$, whenever $A_{\alpha_{n}}(\gamma) \leq K$.

Proof This is a variant of the proof of Lemma 8.2. First note that $A_{\alpha_{n}}(\gamma) \leq K$ implies that $\gamma \subset M^{\prime}$ for sufficiently large $n$. Suppose there is a sequence of finite energy, rational holomorphic maps $F_{n}$ to $\left(\mathbb{R} \times M_{n}, \tilde{J}_{n}\right)$ with one positive end at $\gamma$, where $\widetilde{J}_{n}$ is the almost complex structure for the cobordism given in Lemma 8.3 (called $J_{n}$ there). If $F_{n}$ intersects $\mathbb{R} \times R_{+}\left(\Gamma^{\prime}\right) \times\{0\}$ for all $n$, the proof of Lemma 8.2 produces a holomorphic sphere in $\mathbb{R} \times R_{+}\left(\Gamma^{\prime}\right) \times \mathbb{R}$, a contradiction. (Note that, as $n \rightarrow \infty$, the difference between the almost complex structure $\widetilde{J}_{n}$ and the tailored almost complex structure $J_{n}$ for $\alpha_{n}$ becomes arbitrarily small in the $C^{\infty}$ topology.) Hence $F_{n}$ can be viewed as a map to $\left(\mathbb{R} \times\left(M^{\prime}\right)^{*}, J^{\prime}\right)$ for sufficiently large $n$. Since $J^{\prime}$ is $\mathbb{R}$-invariant, the lemma follows.

Lemma 8.4 implies that the diagram

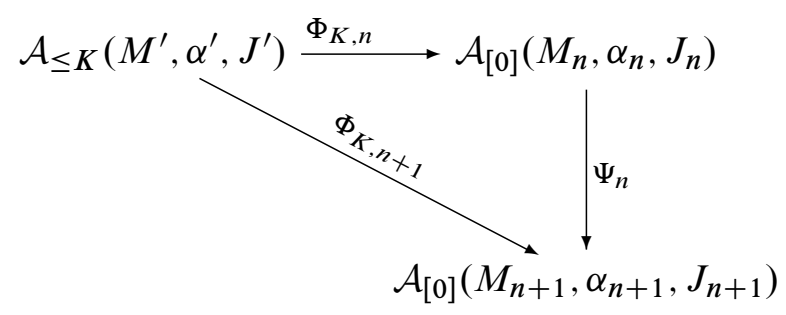

commutes, provided that $n$ is sufficiently large with respect to $K$. 


\subsection{Direct limits}

Definition of $\Phi$ Suppose $n^{\prime} \gg n \gg 0$. By composing $\Psi_{n^{\prime}-1} \circ \Psi_{n^{\prime}-2} \circ \cdots \circ \Psi_{n}$, we obtain a chain map

$$
\Psi_{n, n^{\prime}}: \mathcal{A}_{[0]}\left(M_{n}, \alpha_{n}, J_{n}\right) \rightarrow \mathcal{A}_{[0]}\left(M_{n^{\prime}}, \alpha_{n^{\prime}}, J_{n^{\prime}}\right),
$$

where $\gamma \subset M^{\prime}$ is mapped to $\gamma+\sum_{i} a_{i} \overline{\gamma_{i}}$, the orbits of $\overline{\gamma_{i}}$ are contained in $M^{\prime}$, and $A_{\alpha_{n}}\left(\overline{\gamma_{i}}\right)=A_{\alpha_{n^{\prime}}}\left(\overline{\gamma_{i}}\right)<A_{\alpha_{n}}(\gamma)$. It follows from the commutativity of Diagram (28) that if $K<K^{\prime}$ and if $n$ is sufficiently large with respect to $K^{\prime}$, then the chain map $\Psi_{n, n^{\prime}}$ fits into the following commutative diagram of chain complexes:

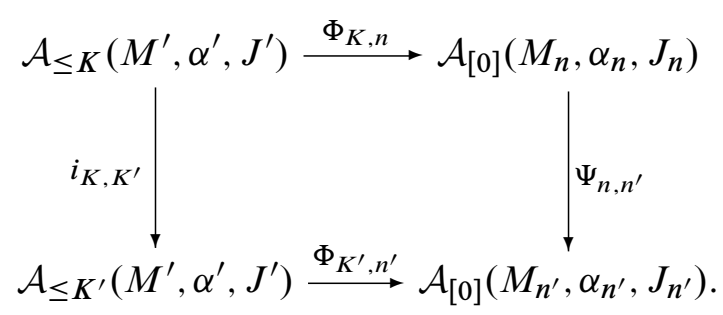

Here $i_{K, K^{\prime}}$ denotes the natural inclusion. Note that the usual chain homotopy argument shows that $\Psi_{n, n^{\prime}}$ is chain homotopic to any continuation map given by a symplectic cobordism from $\alpha_{n}$ to $\alpha_{n^{\prime}}$.

By commutativity of Diagram (29), we can take direct limits to obtain a map

$$
\Phi: \lim _{K \rightarrow \infty} H C_{\leq K}\left(M^{\prime}, \alpha^{\prime}, J^{\prime}\right) \rightarrow \lim _{n \rightarrow \infty} H C_{[0]}\left(M_{n}, \alpha_{n}, J_{n}\right)
$$

on the level of homology. Now observe that

$$
\lim _{K \rightarrow \infty} H C_{\leq K}\left(M^{\prime}, \alpha^{\prime}, J^{\prime}\right)=H C\left(M^{\prime}, \alpha^{\prime}, J^{\prime}\right)=H C\left(M^{\prime}, \Gamma^{\prime}\right),
$$

because the analogous statement on the level of chain complexes holds by definition and taking homology commutes with direct limits. On the other hand,

$$
\lim _{n \rightarrow \infty} H C_{[0]}\left(M_{n}, \alpha_{n}, J_{n}\right)=H C_{[0]}(M, \Gamma),
$$

because the map $\Psi_{n, n^{\prime}}$ induces the canonical isomorphism on homology, so that the direct limit is isomorphic to any single $H C_{[0]}\left(M_{n}, \alpha_{n}, J_{n}\right)$ and is canonically isomorphic to $H C_{[0]}(M, \Gamma)$. We conclude that $\Phi$ defines a map

$$
\Phi: H C\left(M^{\prime}, \Gamma^{\prime}\right) \rightarrow H C_{[0]}(M, \Gamma) .
$$

To complete the proof of Theorem 8.1, we will show that this is an isomorphism. 
In the arguments below, we will use, without further notation, the canonical identifications

$$
\mathcal{A}\left(M^{\prime}, \alpha^{\prime}, J^{\prime}\right) \simeq \mathcal{A}_{[0]}\left(M_{n}, \alpha_{n}, J_{n}\right) \simeq \mathcal{A}_{[0]}\left(M_{n^{\prime}}, \alpha_{n^{\prime}}, J_{n^{\prime}}\right)
$$

arising from the fact that an orbit $\gamma$ in $\mathcal{A}\left(M^{\prime}, \alpha^{\prime}, J^{\prime}\right)$ can naturally be viewed as an orbit in $\mathcal{A}_{[0]}\left(M_{n}, \alpha_{n}, J_{n}\right)$ or in $\mathcal{A}_{[0]}\left(M_{n^{\prime}}, \alpha_{n^{\prime}}, J_{n^{\prime}}\right)$. These are identifications of $\mathbb{Q}$-vector spaces, but not necessarily of chain complexes.

Injectivity of $\Phi$ Refer to Diagram (29). Suppose that $a$ is a cycle in $\mathcal{A}_{\leq K}\left(M^{\prime}, \alpha^{\prime}, J^{\prime}\right)$ and that $a=\partial b$ for some $b \in \mathcal{A}_{[0]}\left(M_{n}, \alpha_{n}, J_{n}\right)$ with $n$ sufficiently large. Then $\Psi_{n, n^{\prime}}$ sends $a \mapsto a$ by Lemma 8.4, and $b \mapsto b+\sum_{i} b_{i}$ by Lemma 8.3, where $A_{\alpha_{n^{\prime}}}\left(b_{i}\right)<$ $A_{\alpha_{n}}(b)$. (Here $A_{\alpha_{n}}(b)$ means the maximum over all the monomials of $b$.) Hence $a=\partial\left(b+\sum_{i} b_{i}\right)$ in $\mathcal{A}_{[0]}\left(M_{n^{\prime}}, \alpha_{n^{\prime}}, J_{n^{\prime}}\right)$. Now, if we let $K^{\prime}>A_{\alpha_{n}}(b)$, then, for sufficiently large $n^{\prime}$, the inclusion $\Phi_{K^{\prime}, n^{\prime}}$ is a chain map by Lemma 8.2. Hence $a=\partial\left(b+\sum_{i} b_{i}\right)$ in $\mathcal{A}_{\leq K^{\prime}}\left(M^{\prime}, \alpha^{\prime}, J^{\prime}\right)$. This proves the injectivity of $\Phi$.

Surjectivity of $\Phi$ Suppose that $a$ is a cycle in $\mathcal{A}_{[0]}\left(M_{n}, \alpha_{n}, J_{n}\right)$ for some $n$. By Lemma 8.4, $\Psi_{n, n^{\prime}}(a)=a+\sum_{i} a_{i}$ stabilizes for sufficiently large $n^{\prime}$. As before, for sufficiently large $K^{\prime}$, the inclusion $\Phi_{K^{\prime}, n^{\prime}}$ is a chain map by Lemma 8.2. Hence $\Phi_{K^{\prime}, n^{\prime}}$ sends $a+\sum_{i} a_{i} \mapsto a+\sum_{i} a_{i}$. This proves the surjectivity of $\Phi$.

\subsection{Proof of Theorem 1.8}

Starting with $\left(M_{i}, \xi_{i}\right)$, let $B_{i}$ be a standard Darboux ball with convex boundary in $M_{i}$, and set $M_{i}^{\prime}=M_{i}-B_{i}$. Applying the convex-to-sutured operation in Lemma 4.1, we obtain sutured contact manifolds $\left(M_{i}^{\prime \prime}, \Gamma_{i}^{\prime \prime}=S^{2 n-1}, U\left(\Gamma_{i}^{\prime \prime}\right), \xi_{i}^{\prime \prime}\right)$, where $R_{ \pm}\left(\Gamma_{i}^{\prime \prime}\right)=D^{2 n}$. We then glue $M_{1}^{\prime \prime}, M_{2}^{\prime \prime}$, and a layer $D^{2 n} \times[-N, N]$ so that $R_{-}\left(\Gamma_{1}^{\prime \prime}\right)$ and $D^{2 n} \times\{N\}$ are identified by a diffeomorphism and $R_{+}\left(\Gamma_{2}^{\prime \prime}\right)$ and $D^{2 n} \times\{-N\}$ are identified by a diffeomorphism. Without loss of generality we may assume that the contact 1-form on $D^{2 n} \times\{-N\}$ has the form $d t+\beta$, and that the contact forms on $M_{i}^{\prime \prime}$ agree with $d t+\beta$. Now observe that all the Reeb orbits of $M^{\prime \prime}=M_{1}^{\prime \prime} \cup M_{2}^{\prime \prime} \cup\left(D^{2 n} \times[-N, N]\right)$ are Reeb orbits of $M_{1}^{\prime \prime}$ or Reeb orbits of $M_{2}^{\prime \prime}$. The rest of the proof of Theorem 1.8(1) is identical to that of Theorem 8.1.

We prove Theorem 1.8(2) using a slightly different argument (which can also be used to give an alternate proof of Theorem 1.8(1)). Let $\left(M^{\prime \prime}, \alpha_{N}\right)$ denote the version of $M^{\prime \prime}$ with neck stretching parameter $N$. (That is, we are using diffeomorphisms to regard the different stretched contact manifolds as different contact forms on the same 3manifold.) Fix almost complex structures $J^{i}$ as needed to define the ECH of $M_{i}^{\prime \prime}$ for $i=1,2$. Let $J_{N}$ be an almost complex structure as needed to define the ECH 
of $\left(M^{\prime \prime}, \alpha_{N}\right)$, which restricts $J^{i}$ on $M_{i}^{\prime \prime}$. An analogue of Lemma 8.2, modified for $\mathrm{ECH}$ as in Proposition 5.20, shows that for any $K$, if $N$ is sufficiently large, then there is a canonical isomorphism

$$
E C H_{\leq K}\left(M^{\prime \prime}, \alpha_{N}, J_{N}\right) \stackrel{\simeq}{\longrightarrow} E C H_{\leq K}\left(M_{1}^{\prime \prime} \sqcup M_{2}^{\prime \prime}\right)
$$

induced by the obvious bijection on generators. From this description of the isomorphism it follows that given $K<K^{\prime}$, if $N$ is sufficiently large, then the above isomorphisms for $K$ and $K^{\prime}$ fit into a commutative diagram

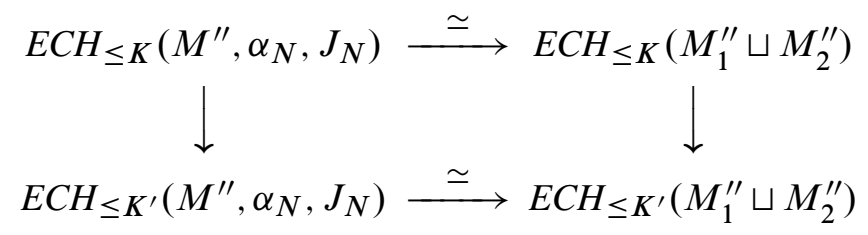

where the vertical arrows are induced by the inclusions of chain complexes.

Now since the Reeb orbits in $M^{\prime \prime}$ and their actions do not depend on the neck stretching parameter $N$, lemmas from [27] can be invoked to show the following:

(i) $E C H_{\leq K}\left(M^{\prime \prime}, \alpha_{N}, J_{N}\right)$ does not depend on $N$, ie for any $N, N^{\prime}$ there is a canonical isomorphism

$$
E C H_{\leq K}\left(M^{\prime \prime}, \alpha_{N}, J_{N}\right) \simeq E C H_{\leq K}\left(M^{\prime \prime}, \alpha_{N^{\prime}}, J_{N^{\prime}}\right) .
$$

Thus we can denote this homology simply by $\operatorname{ECH}_{\leq K}\left(M^{\prime \prime}\right)$.

The above isomorphism is constructed by choosing a generic homotopy $\left\{\left(\alpha_{N(t)}, J_{t}\right) \mid\right.$ $t \in[0,1]\}$ from $\left(\alpha_{N}, J_{N}\right)$ to $\left(\alpha_{N^{\prime}}, J_{N^{\prime}}\right)$, dividing the homotopy into a composition of many short homotopies, and taking the composition of the corresponding continuation isomorphisms from [27]. Note that the latter continuation maps are defined using Seiberg-Witten theory and so are only valid in a closed manifold, but one can extend them to the present situation by embedding into closed manifolds as follows. Given $K$ and $\{N(t)\}$, take an irrational ellipsoid $E$ with its standard contact form which is sufficiently large so that its Reeb orbits have action larger than $K$ and so that it contains a one-parameter family of cylinders $\left\{Z_{t} \mid t \in[0,1]\right\}$ such that the Reeb flow near $\partial Z_{t}$ is diffeomorphic to the Reeb flow near $\left(\partial M^{\prime \prime}, \alpha_{N(t)}\right)$. One can then embed the family of sutured contact manifolds $\left\{\left(M^{\prime \prime}, \alpha_{N(t)}\right) \mid t \in[0,1]\right\}$ into a family of closed contact manifolds by gluing $M^{\prime \prime}$ to $E \backslash Z_{t}$ for each $t$. The desired continuation isomorphism is now obtained by applying [27] to this family of closed contact manifolds. This continuation map still sends $E C H_{\leq K}\left(M^{\prime \prime}, \alpha_{N}, J_{N}\right)$ to $E C H_{\leq K}\left(M^{\prime \prime}, \alpha_{N^{\prime}}, J_{N^{\prime}}\right)$, because the Reeb orbits in $E$ have action greater than $K$. 
(ii) For any given $K$, if $N, N^{\prime}$ are sufficiently large, then the above canonical isomorphism is induced by the obvious bijection on generators. (Here we are again using the $\mathrm{ECH}$ analogue of Lemma 8.2.)

(iii) If $K<K^{\prime}$ then the inclusion-induced map

$$
E C H_{\leq K}\left(M^{\prime \prime}, \alpha_{N}, J_{N}\right) \rightarrow E C H_{\leq K^{\prime}}\left(M^{\prime \prime}, \alpha_{N}, J_{N}\right)
$$

commutes with the canonical isomorphisms in (i) and so induces a well-defined map

$$
E C H_{\leq K}\left(M^{\prime \prime}\right) \rightarrow E C H_{\leq K^{\prime}}\left(M^{\prime \prime}\right) .
$$

It follows from (i) and (ii) that the isomorphism (30) induces a well-defined isomorphism

$$
E C H_{\leq K}\left(M^{\prime \prime}\right) \stackrel{\simeq}{\longrightarrow} E C H_{\leq K}\left(M_{1}^{\prime \prime} \sqcup M_{2}^{\prime \prime}\right) .
$$

By (iii) and Diagram (31), the above isomorphisms fit into a commutative diagram

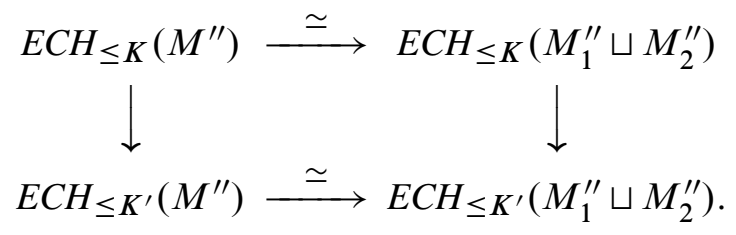

We can then take the direct limit over $K$ to obtain an isomorphism

$$
E C H\left(M^{\prime \prime}\right) \stackrel{\simeq}{\longrightarrow} E C H\left(M_{1}^{\prime \prime} \sqcup M_{2}^{\prime \prime}\right)=E C H\left(M_{1}^{\prime \prime}\right) \otimes E C H\left(M_{2}^{\prime \prime}\right),
$$

where the equality on the right holds because we are using field coefficients. By Theorem 1.6, $E C H\left(M_{i}^{\prime \prime}\right) \simeq \widehat{E C H}\left(M_{i}\right)$ and $E C H\left(M^{\prime \prime}\right) \simeq \widehat{E C H}\left(M_{1} \# M_{2}\right)$. This completes the proof of Theorem 1.8(2).

\section{Second warm-up: neck-stretching in the $\tau$-direction}

Let $\left(M^{\prime}, \Gamma^{\prime}, \alpha^{\prime}\right)$ be a sutured contact manifold and let $(W, \beta)$ be a Liouville cobordism from $\partial_{+} W$ to $\partial_{-} W$, as defined in Example 2.10. Suppose there is a diffeomorphism which takes $\left(\partial R_{+}\left(\Gamma^{\prime}\right), \beta_{0}=\left.\alpha^{\prime}\right|_{\partial R_{+}\left(\Gamma^{\prime}\right)}\right)$ to $\left(\partial_{-} W,\left.\beta\right|_{\partial_{-} W}\right)$. We also assume that $R_{\beta_{0}}$ is nondegenerate. Let us write $N=[0,1] \times[-1,1] \times \Gamma^{\prime}$ with coordinates $(\tau, t, x)$. We construct the interval-fibered extension $\left(M, \Gamma=\partial_{+} W, \alpha\right)$ of $\left(M^{\prime}, \Gamma^{\prime}, \alpha^{\prime}\right)$ as follows: The manifold $M$ is obtained from $M^{\prime} \sqcup N \sqcup(W \times[-1,1])$ by identifying $\{0\} \times[-1,1] \times \Gamma^{\prime} \subset U\left(\Gamma^{\prime}\right)$ and $\{0\} \times[-1,1] \times \Gamma^{\prime} \subset N$ and by identifying $\{1\} \times[-1,1] \times \Gamma^{\prime} \subset N$ and $\partial_{-} W \times[-1,1] \subset W \times[-1,1]$. We then define $\alpha$ as follows:

$$
\alpha= \begin{cases}\alpha^{\prime} & \text { on } M^{\prime}, \\ d t+\bar{\beta} & \text { on } N \cup(W \times[-1,1]),\end{cases}
$$


where $\bar{\beta}$ is a $1-$ form on $W_{N}=\left([0,1] \times \Gamma^{\prime}\right) \cup W$, which equals $e^{\tau} \beta_{0}$ on $[0,1] \times \Gamma^{\prime}$ and $e^{1} \beta$ on $W$.

Let $\kappa>0$. Choose a diffeomorphism

$$
\begin{aligned}
H_{\kappa}:[0,1] \times \Gamma^{\prime} \stackrel{\sim}{\rightarrow}[0, \kappa] \times \Gamma^{\prime}, \\
(\tau, x) \mapsto\left(h_{\kappa}(\tau), x\right),
\end{aligned}
$$

where $h_{\kappa}:[0,1] \stackrel{\sim}{\rightarrow}[0, \kappa], h_{\kappa}(0)=0, h_{\kappa}(1)=\kappa, h_{\kappa}^{\prime}(\tau)=1$ in a neighborhood of $\tau=0,1$, and $h_{\kappa}$ is linear outside a bigger neighborhood of $\tau=0,1$. If $J^{\prime}$ is an almost complex structure on $M^{\prime}$ which is tailored to $\alpha^{\prime}$, then we define its extension $J_{\kappa}$ on $M$ to be tailored to $\alpha$, subject to the following conditions on the projection $\left(J_{\kappa}\right)_{0}$ of $J_{\kappa}$ to $W_{N}$ :

- $\left(J_{\kappa}\right)_{0}$ is independent of $\kappa$ on $W$.

- On $[0,1] \times \Gamma^{\prime},\left(J_{\kappa}\right)_{0}$ is the $H_{\kappa}$-pullback of a $\beta_{0}$-adapted almost complex structure on $[0, \kappa] \times \Gamma^{\prime}$.

By sending $\kappa \rightarrow \infty$, we are "stretching the neck" in the $\tau$-direction.

In this section we prove the following theorem:

Theorem 9.1 An interval-fibered extension $\left(M^{\prime}, \Gamma^{\prime}, \xi^{\prime}\right) \hookrightarrow(M, \Gamma, \xi)$ induces an isomorphism

$$
\Phi: H C\left(M^{\prime}, \Gamma^{\prime}, \xi^{\prime}\right) \stackrel{\sim}{\rightarrow} H C(M, \Gamma, \xi) .
$$

The proof of Theorem 9.1 follows the same outline as the proof of Theorem 8.1.

We first observe that the set of Reeb orbits of $\left(M^{\prime}, \Gamma^{\prime}, \xi^{\prime}, \alpha^{\prime}\right)$ and $(M, \Gamma, \xi, \alpha)$ are the same. The holomorphic curves are restricted by the following analog of Lemma 8.2:

Lemma 9.2 Suppose $\underline{\gamma}^{+}$and $\underline{\gamma}^{-}$consist of orbits in $M^{\prime}$. Then, for sufficiently large $\kappa$,

$$
\mathcal{M}_{g}\left(\underline{\gamma}^{+} ; \underline{\gamma^{-}} ; \mathbb{R} \times\left(M^{\prime}\right)^{*}, J^{\prime}\right)=\mathcal{M}_{g}\left(\underline{\gamma}^{+} ; \underline{\gamma}^{-} ; \mathbb{R} \times M^{*}, J_{\kappa}\right)
$$

Proof Arguing by contradiction, suppose there is a sequence of holomorphic curves

$$
F_{\kappa}=\left(a_{\kappa}, f_{\kappa}\right):\left(\Sigma_{\kappa}, j_{\kappa}, \mathbf{m}_{\kappa}\right) \rightarrow\left(\mathbb{R} \times M^{*}, J_{\kappa}\right)
$$

in $\mathcal{M}_{g}\left(\gamma^{+} ; \gamma^{-} ; \mathbb{R} \times M^{*}, J_{\kappa}\right)$, whose second component $f_{\kappa}$ nontrivially intersects $\left(W_{N}\right)^{\mathrm{TB}}=\mathbb{R} \times W_{N}$ for all $\kappa$. (Here the superscript TB indicates that we are extending towards the top and bottom.) We write $f_{\kappa}=\left(b_{\kappa}, v_{\kappa}\right)$ when $f_{\kappa}(x) \in\left(W_{N}\right)^{\mathrm{TB}}$; here $b_{\kappa}=t \circ f_{\kappa}$ and $v_{\kappa}$ is the projection onto $W_{N}$. 
On $\mathbb{R} \times M$ we use the Riemannian metric

$$
g_{\kappa}=d s \otimes d s+\alpha \otimes \alpha+\omega\left(\cdot, J_{\kappa} \cdot\right)-\omega\left(J_{\left.\kappa^{\cdot}, \cdot\right),}\right.
$$

where $\omega$ is the (not everywhere closed) 2 -form defined by

$$
\omega= \begin{cases}d \alpha^{\prime} & \text { on } M^{\prime}, \\ d \tilde{\tau} \wedge \beta_{0}+d \beta_{0} & \text { on } H_{\kappa}\left([0,1] \times \Gamma^{\prime}\right)=[0, \kappa] \times \Gamma^{\prime}, \\ d \beta & \text { on } W .\end{cases}
$$

Here $\tilde{\tau}$ is the coordinate on $[0, \kappa]$.

If there is a gradient blow-up for the sequence $F_{\kappa}$ in the neck region $\mathbb{R} \times \mathbb{R} \times[0, \kappa] \times \Gamma^{\prime}$, then the usual argument gives us a nonconstant finite energy plane in $\mathbb{R} \times \mathbb{R} \times \mathbb{R} \times \Gamma^{\prime}$. However, since there are no closed orbits in $\mathbb{R} \times \mathbb{R} \times \mathbb{R} \times \Gamma^{\prime}$, we obtain a contradiction. Putting in finitely many punctures on $\Sigma_{\kappa}-\mathbf{m}_{\kappa}$ to bound the gradient of $F_{\kappa}$ on $\dot{\Sigma}_{\kappa}=\Sigma_{\kappa}-\left(\mathbf{m}_{\kappa} \cup \mathbf{m}_{\kappa}^{0}\right)$ as usual, we apply similar considerations as in Proposition 5.18. There is a connected subsurface $\bar{\Sigma}_{\kappa}$ of $\dot{\Sigma}_{\kappa}$ which satisfies the following:

- $f_{\kappa}\left(\bar{\Sigma}_{\kappa}\right) \subset \mathbb{R} \times\left(\left(\left[\frac{1}{3}, 1\right] \times \Gamma^{\prime}\right) \cup W\right)$.

- $\bar{\Sigma}_{\kappa}$ is a union of type $A \cup B$, where $A$ is a possibly empty union of thick and thin components of $\dot{\Sigma}_{\kappa}$ and $B$ is a nonempty union of annular subsets of thin components of $\dot{\Sigma}_{\kappa}$.

- The components of $B$ have the form $E \times S^{1}$ inside thin components $\left[-R, R^{\prime}\right] \times S^{1}$ or $[-R, \infty) \times S^{1}$, where $E$ is an interval in $\left[-R, R^{\prime}\right]$ or $[-R, \infty)$ and length $(E)$ tends to $\infty$ as $\kappa \rightarrow \infty$.

- $f_{\kappa}\left(\bar{\Sigma}_{\kappa}\right)$ nontrivially intersects $\mathbb{R} \times W$ and $f\left(\partial \bar{\Sigma}_{\kappa}\right) \subset \mathbb{R} \times\left[\frac{1}{3}, \frac{2}{3}\right] \times \Gamma^{\prime}$.

We now consider $v_{\kappa}$ restricted to $\bar{\Sigma}_{\kappa}$. Observe that the finiteness of the $d \alpha$-energy of $F_{\kappa}$ implies the finiteness of $d \bar{\beta}$-energy of $v_{\kappa}$. Moreover, if $\widetilde{\beta}=f(\tau) \beta_{0}$ on $[0,1] \times \Gamma^{\prime}$, where $f:[0,1] \rightarrow \mathbb{R}$ is a smooth, monotonically increasing function which agrees with $e^{\tau}$ on $\left[0, \frac{2}{3}\right]$ and satisfies $f(1)=e^{1}$, then Stokes' theorem gives an upper bound on the $d \widetilde{\beta}$-energy of $v_{\kappa}$ on $\left[\frac{2}{3}, 1\right] \times \Gamma^{\prime}$. We then have the Hofer energy bound of $v_{\kappa}$ on $\left[\frac{2}{3}, 1\right] \times \Gamma^{\prime}$. Therefore, $v_{\kappa}$ converges to a finite energy holomorphic curve in $W \cup\left(\mathbb{R} \times \Gamma^{\prime}\right)$ without any positive ends, contradicting Stokes' theorem. (Here the $\mathbb{R}$-coordinate corresponds to $\tilde{\tau}$.) Hence, for sufficiently large $\kappa, F_{\kappa}$ does not intersect $\mathbb{R} \times \mathbb{R} \times W$. It follows that $F_{\kappa}$ has image inside $\mathbb{R} \times\left(M^{\prime}\right)^{*}$.

By Lemma 9.2, given $K>0$, there exists $\kappa>0$ such that all the punctured holomorphic spheres in $\left(\mathbb{R} \times M^{*}, J_{\kappa}\right)$ which are asymptotic to $\gamma \in \mathcal{A}_{\leq K}\left(M^{\prime}, \alpha^{\prime}, J^{\prime}\right)$ at the positive 
end are disjoint from $\mathbb{R} \times W \times[-1,1]$. Hence we have an inclusion of chain complexes

$$
\Phi_{K, \kappa}: \mathcal{A}_{\leq K}\left(M^{\prime}, \alpha^{\prime}, J^{\prime}\right) \hookrightarrow \mathcal{A}\left(M, \alpha, J_{\kappa}\right),
$$

for sufficiently large $\kappa$.

We now compare $\left(M, \alpha, J_{\kappa}\right)$ and $\left(M, \alpha, J_{\kappa+1}\right)$ for sufficiently large $\kappa$. Observe that the contact forms are the same, and we are only interpolating between $J_{\kappa}$ and $J_{\kappa+1}$. The almost complex structures differ only on $\mathbb{R} \times \mathbb{R} \times[0,1] \times \Gamma^{\prime}$. We identify $H_{\kappa}:[0,1] \times \Gamma^{\prime} \stackrel{\sim}{\rightarrow}[0, \kappa] \times \Gamma^{\prime}$ and use coordinates $\tilde{\tau}$ on $[0, \kappa]$. Then $\left(J_{\kappa}\right)_{0}$ and $\left(J_{\kappa+1}\right)_{0}$ agree on $\operatorname{ker} \beta_{0}$; however, $\left(J_{\kappa}\right)_{0}$ sends $\partial_{\tilde{\tau}} \mapsto R_{\beta_{0}}$ and $\left(J_{\kappa+1}\right)_{0}$ sends $\partial_{\tilde{\tau}} \mapsto f(\tilde{\tau}) R_{\beta_{0}}$, where we may take $1-2 / \kappa \leq f(\tilde{\tau}) \leq 1$. Let $\left(J_{\kappa+1-s}\right)_{0}, s \in[0,1]$, be an interpolation between $\left(J_{\kappa+1}\right)_{0}$ and $\left(J_{\kappa}\right)_{0}$ where only the function $f(\tilde{\tau})$ is varying. Now define the almost complex structure $J_{\kappa+1-s}$ on $M$ to be tailored to $\alpha$ so that the projection to $W_{N}$ is $\left(J_{\kappa+1-s}\right)_{0}$. We then define the almost complex structure $\widetilde{J}_{\kappa}$ on $\mathbb{R} \times M^{*}$ so that

(1) $\left.\left(\widetilde{J}_{\kappa}\right)\right|_{s \geq 1}=J_{\kappa}$ and $\left.\left(\widetilde{J}_{\kappa}\right)\right|_{s \leq 0}=J_{\kappa+1}$;

(2) $\left.\left(\tilde{J}_{\kappa}\right)\right|_{s}=J_{\kappa+1-s}$.

The following is the analog of Lemma 8.3:

Lemma 9.3 The cobordism $\left(\mathbb{R} \times M^{*}, \widetilde{J}_{\kappa}\right)$ gives rise to a continuation map

$$
\Psi_{\kappa}: \mathcal{A}\left(M, \alpha, J_{\kappa}\right) \rightarrow \mathcal{A}\left(M, \alpha, J_{\kappa+1}\right)
$$

with the property that, if $\gamma \subset M^{\prime}$, then

$$
\gamma \mapsto \gamma+\sum_{i} a_{i} \overrightarrow{\gamma_{i}}
$$

where all the orbits of $\overrightarrow{\gamma_{i}}$ are contained in $M^{\prime}$ and $A(\gamma)>A\left(\overrightarrow{\gamma_{i}}\right)=A_{\alpha}\left(\overrightarrow{\gamma_{i}}\right)$.

Proof This is straightforward, since both $J_{\kappa}$ and $J_{\kappa+1}$ are adapted to $\alpha$. We easily see that $\omega=d(g(s) \alpha)$ is $\widetilde{J}_{\kappa}$-nonnegative whenever $g(s)$ is a positive, monotonically increasing function.

We also have the following lemma:

Lemma 9.4 Given $K>0$, there exists $\kappa_{0}>0$ such that for all $\kappa \geq \kappa_{0}$,

$$
\Psi_{\kappa}: \mathcal{A}\left(M, \alpha, J_{\kappa}\right) \rightarrow \mathcal{A}\left(M, \alpha, J_{\kappa+1}\right)
$$

maps $\gamma \mapsto \gamma$, whenever $A_{\alpha}(\gamma) \leq K$. 
Proof Similar to that of Lemma 9.2, with one difference: If there is a sequence of holomorphic curves

$$
F_{\kappa}=\left(a_{\kappa}, f_{\kappa}\right):\left(\Sigma_{\kappa}, j_{\kappa}, \mathbf{m}_{\kappa}\right) \rightarrow\left(\mathbb{R} \times M^{*}, \tilde{J}_{\kappa}\right)
$$

in $\mathcal{M}_{g}\left(\gamma^{+} ; \gamma^{-} ; \mathbb{R} \times M^{*}, \tilde{J}_{\kappa}\right)$, then there is a restriction of $F_{\kappa}$ to a connected subsurface $\bar{\Sigma}_{\kappa}$ as before, whose image is contained in $\left(W_{N}\right)^{\mathrm{TB}}$. If we write $f_{\kappa}=\left(b_{\kappa}, v_{\kappa}\right)$, then each $v_{\kappa}$ is not necessarily $\left(J_{\kappa}\right)_{0}-$ or $\left(J_{\kappa+1}\right)_{0}$-holomorphic. However, since the sequence $v_{\kappa} \mid \bar{\Sigma}_{\kappa}$ limits to a holomorphic curve in $W \cup\left(\mathbb{R} \times \Gamma^{\prime}\right)$, after possibly taking a subsequence, the proof of Lemma 9.2 still carries over. (Compare Section 6.2.)

Putting Lemmas 9.2, 9.3 and 9.4 together, the direct limit argument in Section 8.3 proves Theorem 9.1.

\section{Proof of Theorem 1.9}

In this section we prove Theorem 1.9, ie the inclusion map under sutured manifold gluing. The proof is a combination of the previous two sections.

\subsection{Stretching the neck}

Keeping the notation from Section 4.3, the main theorem of this section is the following:

Theorem 10.1 Suppose the orbits of $\underline{\gamma}^{+}$and $\underline{\gamma}^{-}$are contained in $M^{\prime}$. Then there exist $\kappa>0$ and $n_{0}=n_{0}(\kappa)>0$ such that the tailored almost complex structure $J_{\kappa}^{\prime}$ on $\left(M^{\prime}\right)^{*}$ satisfies

$$
\mathcal{M}_{g}\left(\underline{\gamma}^{+} ; \underline{\gamma}^{-} ; \mathbb{R} \times\left(M^{\prime}\right)^{*}, J_{\kappa}^{\prime}\right)=\mathcal{M}_{g}\left(\underline{\gamma}^{+} ; \underline{\gamma}^{-} ; \mathbb{R} \times M_{n}^{*}, J_{\kappa, n}\right),
$$

for all $n \geq n_{0}$.

Proof We analyze the convergence of a sequence of finite energy holomorphic maps

$$
F_{n}=\left(a_{n}, f_{n}\right):\left(\Sigma_{n}, j_{n}, \mathbf{m}_{n}\right) \rightarrow\left(\mathbb{R} \times M_{n}^{*}, J_{\kappa, n}\right)
$$

in $\mathcal{M}_{g}\left(\gamma^{+} ; \gamma^{-} ; \mathbb{R} \times M_{n}^{*}, J_{\kappa, n}\right)$. Our first reduction is to restrict the range of $F_{n}$ from $\mathbb{R} \times M_{n}^{*}$ to $\overline{\mathbb{R}} \times M_{n}^{(1)}$. Indeed, by Remark 4.9, any holomorphic map $F_{n}$ is disjoint from $\mathbb{R} \times V^{*}$. From now on, we consider the sequence

$$
F_{n}:\left(\Sigma_{n}, j_{n}, \mathbf{m}_{n}\right) \rightarrow\left(\mathbb{R} \times M_{n}^{(1)}, J_{\kappa, n}\right) .
$$

Recall that $M_{e}^{\prime}$ is the infinite interval-fibered extension of $M^{\prime}$, obtained from $M^{\prime}$ by attaching an interval bundle $S^{\prime} \times I$ over $S^{\prime}=S_{\infty}-S$ (as given in Equations (13) 
and (14)), and that $\left(M_{e}^{\prime}\right)^{\mathrm{TB}}$ is the partial completion of $M_{e}^{\prime}$, obtained by attaching just the Top and the Bottom. The theorem now follows from combining the following Lemma 10.2 and Lemma 10.3.

Lemma 10.2 For sufficiently large $\kappa>0$, the almost complex structure $J_{\kappa}^{\prime}$ tailored to $\left(M^{\prime}\right)^{*}$ satisfies

$$
\mathcal{M}_{g}\left(\underline{\gamma}^{+} ; \underline{\gamma}^{-} ; \mathbb{R} \times\left(M^{\prime}\right)^{*}, J_{\kappa}^{\prime}\right)=\mathcal{M}_{g}\left(\underline{\gamma}^{+} ; \underline{\gamma}^{-} ; \mathbb{R} \times\left(M_{e}^{\prime}\right)^{\mathrm{TB}}, J_{\kappa, n}\right) .
$$

Observe that, by the construction in Section 4.3, the almost complex structure $J_{\kappa, n}$ does not depend on $n$ when restricted to $M_{e}^{\prime}$.

Proof of Lemma 10.2 Similar to that of Lemma 9.2. The only difference is that the region $S^{\prime} \cup\left([-1,0] \times \Gamma^{\prime}\right)$ analogous to $W$ is not compact, since $M_{e}^{\prime}$ is an infinite interval-fibered extension of $M^{\prime}$. Hence the sequence

$$
v_{\kappa}: \bar{\Sigma}_{\kappa} \rightarrow S^{\prime} \cup\left([-1,0] \times \Gamma^{\prime}\right)
$$

may not converge, since $v_{\kappa}$ can be pushed towards the ends of $S^{\prime}$. However, most of the analysis in [3, Section 10] can be carried out for the portion of $\bar{\Sigma}_{\kappa}$ mapped into $[-1,0] \times \Gamma^{\prime}$ by $v_{\kappa}$. In particular there must be a finite set of disjoint separating curves in $\bar{\Sigma}_{\kappa}$ which converge to some Reeb orbits as negative punctures. We can assume without loss of generality that those curves are $\partial \bar{\Sigma}_{\kappa}$. Hence, for sufficiently large $\kappa$, $\int_{\partial \bar{\Sigma}_{\kappa}} v_{\kappa}^{*} \beta<0$ (the negative sign because $\partial \bar{\Sigma}_{\kappa}$ approaches a Reeb orbit as a negative puncture). Then $\int_{\bar{\Sigma}_{\kappa}} v_{\kappa}^{*} d \beta<0$ by Stokes' theorem, contradicting the positivity of the symplectic area on holomorphic curves.

Lemma 10.3 Given $\kappa>0$, there exists $n_{0}>0$ so that for all $n \geq n_{0}$,

$$
\mathcal{M}_{g}\left(\underline{\gamma}^{+} ; \underline{\gamma}^{-} ; \mathbb{R} \times M_{n}^{(1)}, J_{\kappa, n}\right)=\mathcal{M}_{g}\left(\underline{\gamma}^{+} ; \underline{\gamma}^{-} ; \mathbb{R} \times\left(M_{e}^{\prime}\right)^{\mathrm{TB}}, J_{\kappa, n}\right) .
$$

Proof of Lemma 10.3 Take a sequence $F_{n} \in \mathcal{M}_{g}\left(\underline{\gamma}^{+} ; \underline{\gamma^{-}} ; \mathbb{R} \times M_{n}^{(1)}, J_{\kappa, n}\right)$. If $A$ and $B$ are subsets of a metric space $(X, d)$, we define the distance from $A$ to $B$ to be $\sup _{x \in A} d(x, B)$. This "distance" is not symmetric, but that is not a problem. We apply the argument in Proposition 5.18 and Lemma 8.2 to bound the distance from $\operatorname{Im}\left(F_{n}\right)$ to the interval-fibered extension $\left(M_{e}^{\prime}, \alpha_{n}, J_{\kappa, n}\right)$. Although the interval-fibered extension is noncompact, $\mathbb{R} \times M_{e}^{\prime}$ has bounded geometry due to the fact that the almost complex structures on the pieces $P_{+}^{c} \times[2 k n-1,2 k n+1]$ are isomorphic (and similarly for $\left.P_{-}^{c} \times[-2 k n-1,-2 k n+1]\right)$, so we can use the same compactness arguments of Proposition 5.18 and Lemma 8.2. 
The ECH case We have the following analog of Theorem 10.1 in the ECH case:

Theorem 10.4 Let $\left\{\left(\gamma_{i}, m_{i}\right)\right\}$ and $\left.\left\{\gamma_{j}^{\prime}, m_{j}^{\prime}\right)\right\}$ be orbit sets in $M^{\prime}$. Then there is some $n_{0} \in \mathbb{N}$ and some tailored almost complex structure $J^{\prime}$ on $\left(M^{\prime}\right)^{*}$ such that all flow lines in $\left(\mathbb{R} \times M_{n}^{*}, J_{n}\right)$ from $\left\{\left(\gamma_{i}, m_{i}\right)\right\}$ to $\left.\left\{\gamma_{j}^{\prime}, m_{j}^{\prime}\right)\right\}$ are contained in $\left(\mathbb{R} \times\left(M^{\prime}\right)^{*}, J^{\prime}\right)$ for all $n \geq n_{0}$.

Proof The proof of Theorem 10.4 is similar to that of Proposition 5.20. We can restrict to $\left(\mathbb{R} \times M_{n}^{(1)}, J_{\kappa, n}\right)$ as in the contact homology case, and apply the GromovTaubes compactness theorem in dimension four to bound the distances of $\operatorname{Im}\left(F_{n}\right)$ to $\left(M_{n}^{(2)}, \alpha_{n}^{(2)}, J_{\kappa, n}\right)$ and $\left(M_{e}^{\prime}, \alpha_{n}, J_{\kappa, n}\right)$.

The analog of Lemma 10.2 is straightforward and does not involve $\kappa$ since $\operatorname{dim} M^{\prime}=3$ and the projection of $J^{\prime}$ to $J_{0}^{\prime}$ on $S_{\infty}$ makes $S_{\infty}$ into a Riemann surface: Let $F$ be a holomorphic map to $\mathbb{R} \times\left(M_{e}^{\prime}\right)^{\mathrm{TB}}$, whose ends are contained in $\mathbb{R} \times\left(M^{\prime}\right)^{*}$. Also let $S^{\prime \prime}=\left(S_{\infty}-S\right) \cup\left([-1,0] \times \Gamma^{\prime}\right)$. Then consider the restriction of $F$ to $\mathbb{R} \times S^{\prime \prime} \times \mathbb{R}$, composed with the projection to $S^{\prime \prime}$. It is a holomorphic map between Riemann surfaces, and hence is an open mapping; on the other hand it is also proper. We now obtain a contradiction since $S^{\prime \prime}$ is noncompact. We conclude that $F$ does not intersect $\mathbb{R} \times S^{\prime \prime} \times \mathbb{R}$.

\subsection{Continuation maps and direct limits}

In this section we prove part of Theorem 1.9, namely we define the map

$$
\Phi: H C\left(M^{\prime}, \alpha^{\prime}\right) \rightarrow H C(M, \alpha)
$$

and show that $\Phi$ is injective.

By Theorem 10.1, given $K>0$, there are $\kappa>0$ and $n_{0}(\kappa)>0$ such that for all $n \geq n_{0}(\kappa)$ there is an inclusion of chain complexes

$$
\Phi_{K, \kappa, n}: \mathcal{A}_{\leq K}\left(M^{\prime}, \alpha^{\prime}, J_{\kappa}^{\prime}\right) \hookrightarrow \mathcal{A}\left(M_{n}, \alpha_{n}, J_{\kappa, n}\right) .
$$

The following lemma is essentially the same as the combination of Lemmas 8.3 and 8.4 - the only difference is the bounded geometry of the interval-fibered portion - and its proof will be omitted.

Lemma 10.5 Given $K>0$ and $\kappa>0$, there exists $n_{0}(\kappa)>0$ such that for all $n \geq n_{0}(\kappa)$ there is a cobordism $\left(\mathbb{R} \times M_{n}^{*}, J\right)$ which gives rise to a continuation map

$$
\Psi_{n}: \mathcal{A}\left(M_{n}, \alpha_{n}, J_{\kappa, n}\right) \rightarrow \mathcal{A}\left(M_{n+1}, \alpha_{n+1}, J_{\kappa, n+1}\right),
$$

with the following properties: 
(1) If $A_{\alpha_{n}}(\gamma) \leq K$, then $\Psi_{n}(\gamma)=\gamma$.

(2) If $\gamma \subset M^{\prime}$, then $\Psi_{n}(\gamma)=\gamma+\sum_{i} a_{i} \overrightarrow{\gamma_{i}}$, where all the orbits of $\overrightarrow{\gamma_{i}}$ are contained in $M^{\prime}$ and $A_{\alpha_{n}}(\gamma)>A_{\alpha_{n+1}}\left(\vec{\gamma}_{i}\right)$.

It follows that given $K>0$ there exist $\kappa>0$ and $n_{0}(\kappa)>0$ such that if $n \geq n_{0}(\kappa)$, then the following diagram of chain complexes commutes:

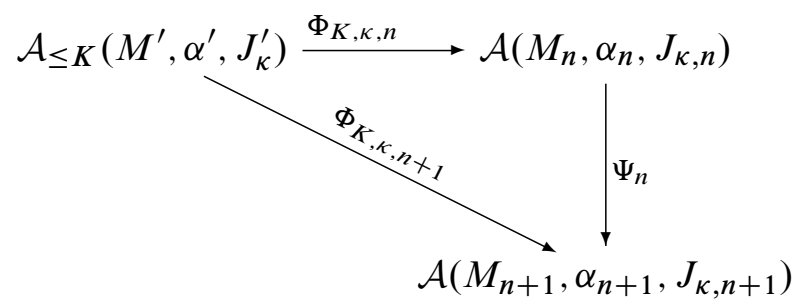

Next consider the continuation maps

$$
\begin{aligned}
i_{\kappa, \kappa+1}: \mathcal{A}\left(M^{\prime}, \alpha^{\prime}, J_{\kappa}^{\prime}\right) & \rightarrow \mathcal{A}\left(M^{\prime}, \alpha^{\prime}, J_{\kappa+1}^{\prime}\right), \\
j_{\kappa, \kappa+1}: \mathcal{A}\left(M_{n}, \alpha_{n}, J_{\kappa, n}\right) & \rightarrow \mathcal{A}\left(M_{n}, \alpha_{n}, J_{\kappa+1, n}\right),
\end{aligned}
$$

which are defined as in Lemma 9.3. The map $i_{\kappa, \kappa+1}$ sends $\gamma \mapsto \gamma+\sum_{i} a_{i} \overrightarrow{\gamma_{i}}$, where $A_{\alpha^{\prime}}(\gamma)>A_{\alpha^{\prime}}\left(\overrightarrow{\gamma_{i}}\right)$. This is due to the fact that the contact form $\alpha^{\prime}$ is the same for the domain and the range. Similar considerations hold for $j_{\kappa, \kappa+1}$. We then have the following lemma:

Lemma 10.6 Given $K^{\prime}>K>0$, there exists $\kappa_{0}>0$ such that for all $\kappa \geq \kappa_{0}$ there exists $n(\kappa)$ such that for all $n \geq n(\kappa)$, the following diagram commutes:

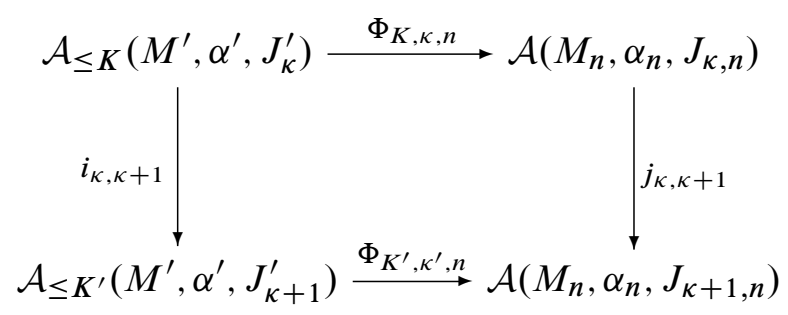

Moreover, if $\gamma \in \mathcal{A}_{\leq K}\left(M^{\prime}, \alpha^{\prime}, J_{\kappa}^{\prime}\right)$, then all the maps in the diagram send $\gamma \mapsto \gamma$.

Proof The proof is similar to that of Lemma 9.4. For sufficiently large $\kappa$, if $A_{\alpha^{\prime}}(\gamma) \leq K$, then $i_{\kappa, \kappa+1}(\gamma)=\gamma$. The same holds for $j_{\kappa, \kappa+1}$, provided we choose $n$ to be sufficiently large in response to $\kappa$. 
Definition of $\Phi$ Suppose $n^{\prime}>n>0$. By composing $\Psi_{n^{\prime}-1} \circ \Psi_{n^{\prime}-2} \circ \cdots \circ \Psi_{n}$, we obtain a chain map

$$
\Psi_{n, n^{\prime}}: \mathcal{A}\left(M_{n}, \alpha_{n}, J_{\kappa, n}\right) \rightarrow \mathcal{A}\left(M_{n^{\prime}}, \alpha_{n^{\prime}}, J_{\kappa, n^{\prime}}\right),
$$

where $\gamma \subset M^{\prime}$ is mapped to $\gamma+\sum_{i} a_{i} \overrightarrow{\gamma_{i}}$ with orbits of $\overrightarrow{\gamma_{i}}$ contained in $M^{\prime}$ and $A_{\alpha_{n^{\prime}}}\left(\overrightarrow{\gamma_{i}}\right)<A_{\alpha_{n}}(\gamma)$. Similarly, if $\kappa^{\prime}>\kappa>0$, then we can define $i_{\kappa, \kappa^{\prime}}$ and $j_{\kappa, \kappa^{\prime}}$ by composing chain maps of type $i_{\kappa, \kappa+1}$ and $j_{\kappa, \kappa+1}$. Given $K^{\prime}>K>0$, there exists $\kappa_{0}$ such that if $\kappa^{\prime}>\kappa \geq \kappa_{0}$ and $n \geq n\left(\kappa, \kappa^{\prime}\right)$, then the chain maps $\Psi_{n, n^{\prime}}, i_{\kappa, \kappa^{\prime}}$, and $j_{\kappa, \kappa^{\prime}}$ fit into the following commutative diagram of chain complexes:

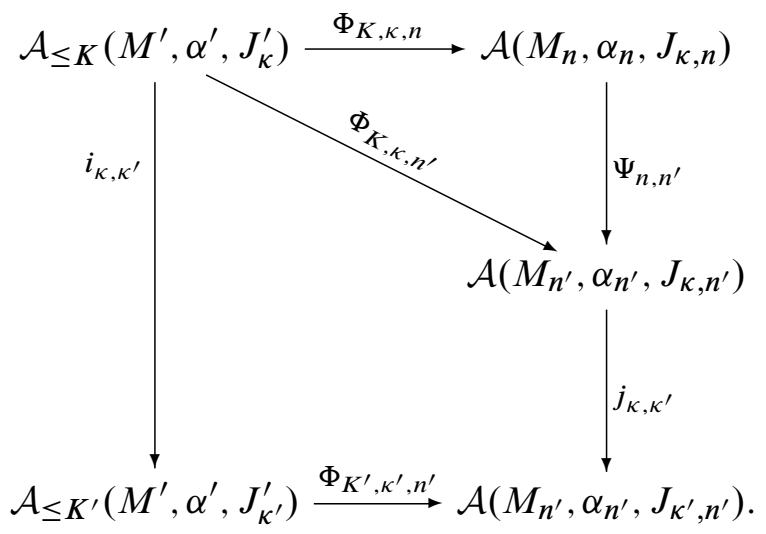

Now,

$$
H C\left(M^{\prime}, \alpha^{\prime}\right)=\lim _{K \rightarrow \infty} H C_{\leq K}\left(M^{\prime}, \alpha^{\prime}, J_{\kappa(K)}^{\prime}\right),
$$

since the contact form $\alpha^{\prime}$ does not vary while $K \rightarrow \infty$. The diagram induces the map

$$
\Phi: H C\left(M^{\prime}, \alpha^{\prime}\right) \rightarrow \lim _{\kappa \rightarrow \infty} H C\left(M_{n(\kappa)}, \alpha_{n(\kappa)}, J_{\kappa, n(\kappa)}\right)
$$

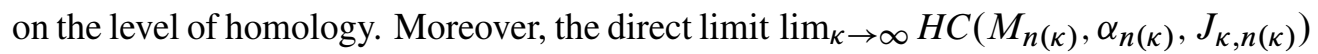
is isomorphic to any single $H C\left(M_{n(\kappa)}, \alpha_{n(\kappa)}, J_{\kappa, n(\kappa)}\right)$.

Injectivity of $\Phi$ Refer to Diagram (36). Suppose $a$ is a cycle in $\mathcal{A}_{\leq K}\left(M^{\prime}, \alpha^{\prime}, J_{\kappa}^{\prime}\right)$ and $a=\partial b$ for some $b \in \mathcal{A}\left(M_{n}, \alpha_{n}, J_{\kappa, n}\right)$ with $n$ sufficiently large. Note that for homological reasons, all the orbits of $b$ must be contained in $M^{\prime}$. Then $\Psi_{n, n^{\prime}}$ sends $a \mapsto a$ and $b \mapsto b+\sum_{i} b_{i}$ by Lemma 10.5, where all the orbits of $b_{i}$ are contained in $M^{\prime}$ and $A_{\alpha_{n^{\prime}}}\left(b_{i}\right)<A_{\alpha_{n}}(b)$, where the latter means the maximum over all the monomials of $b$. Hence $a=\partial\left(b+\sum_{i} b_{i}\right)$ in $\mathcal{A}\left(M_{n^{\prime}}, \alpha_{n^{\prime}}, J_{\kappa, n^{\prime}}\right)$. For sufficiently large $n^{\prime}$, if we apply $j_{\kappa, \kappa^{\prime}}$ to $a=\partial\left(b+\sum_{i} b_{i}\right)$, we obtain $a=\partial\left(b+\sum_{i} b_{i}^{\prime}\right)$ in $\mathcal{A}\left(M_{n^{\prime}}, \alpha_{n^{\prime}}, J_{\kappa^{\prime}, n^{\prime}}\right)$ with $A_{\alpha_{n^{\prime}}}\left(b_{i}^{\prime}\right)<A_{\alpha_{n^{\prime}}}(b)$. Now, if we let $K^{\prime}>A_{\alpha_{n^{\prime}}}(b)$, then there is a sufficiently large $n^{\prime}$ such that the map $\Phi_{K^{\prime}, \kappa^{\prime}, n^{\prime}}$ is injective by Theorem 10.1 . Hence $a=\partial\left(b+\sum_{i} b_{i}^{\prime}\right)$ in $\mathcal{A}_{\leq K^{\prime}}\left(M^{\prime}, \alpha^{\prime}, J_{\kappa^{\prime}}^{\prime}\right)$. This proves the injectivity of $\Phi$. 


\subsection{The inclusion map is well-defined}

In this section we prove that the inclusion map

$$
\Phi: H C\left(M^{\prime}, \Gamma^{\prime}, \xi^{\prime}\right) \rightarrow H C(M, \Gamma, \xi)
$$

does not depend on the choices made to define it. By this we mean the following:

Proposition 10.7 Let $\left(\alpha^{\prime}\right)^{0}$ and $\left(\alpha^{\prime}\right)^{1}$ be two contact forms which are adapted to the sutured contact manifold $\left(M^{\prime}, \Gamma^{\prime}, U\left(\Gamma^{\prime}\right), \xi^{\prime}\right)$, and let $\alpha_{n}^{0}, \alpha_{n}^{1}$ be their extensions to $M_{n}$. Then there is a commutative diagram

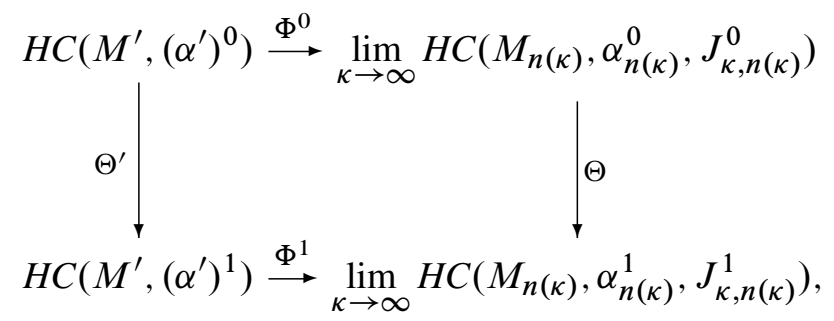

where the $\Phi^{i}$ are the inclusion maps defined in Section 10.2 and $\Theta, \Theta^{\prime}$ are the continuation maps given in Section 6.2.

Proof Let $\left(\alpha^{\prime}\right)^{0}$ and $\left(\alpha^{\prime}\right)^{1}$ be two contact forms adapted to $\left(M^{\prime}, \Gamma^{\prime}, U\left(\Gamma^{\prime}\right), \xi^{\prime}\right)$, and let $\alpha_{n}^{0}$ and $\alpha_{n}^{1}$ be their extensions to $M_{n}$. Also let $\left(J_{\kappa}^{\prime}\right)^{0}$ and $\left(J_{\kappa}^{\prime}\right)^{1}$ be the almost complex structures on $M^{\prime}$ corresponding to $\left(\alpha^{\prime}\right)^{0}$ and $\left(\alpha^{\prime}\right)^{1}$, as defined in Section 4.3, and let $J_{\kappa, n}^{0}$ and $J_{\kappa, n}^{1}$ be their extensions to $M_{n}$. Also write $\left(\beta^{\prime}\right)_{0}^{i}=\left.\left(\alpha^{\prime}\right)^{i}\right|_{\partial R_{+}}\left(\Gamma^{\prime}\right)$ and $\left(\beta^{\prime}\right)^{i}=\left.\left(\alpha^{\prime}\right)^{i}\right|_{R_{+}}\left(\Gamma^{\prime}\right)$.

Since $\left(\alpha^{\prime}\right)^{0}$ and $\left(\alpha^{\prime}\right)^{1}$ are contact forms for the same contact structure $\xi^{\prime}$, we can write $\left(\alpha^{\prime}\right)^{0}=f \cdot\left(\alpha^{\prime}\right)^{1}$, where $f$ is constant in a neighborhood of the sutures. Moreover, we can write $\left(\beta^{\prime}\right)_{0}^{0}=C\left(\beta^{\prime}\right)_{0}^{1}$ for some constant $C$, which we take to be equal to 1 for simplicity. Also, if we identify the manifolds $M_{n}$ using the appropriate diffeomorphisms, then we can write $\alpha_{n}^{0}=f_{n} \alpha_{n}^{1}$.

Choose a 1 -parameter family $f^{\rho}, \rho \in[0,1]$, where $f^{0}=f$ and $f^{1}=1$. We then use the family $f^{\rho}\left(\alpha^{\prime}\right)^{1}$ to construct a symplectic cobordism and an almost complex structure as in Section 6.2 and to define a continuation map

$$
\Theta_{\kappa}^{\prime}: \mathcal{A}\left(M^{\prime},\left(\alpha^{\prime}\right)^{0},\left(J_{\kappa}^{\prime}\right)^{0}\right) \rightarrow \mathcal{A}\left(M^{\prime},\left(\alpha^{\prime}\right)^{1},\left(J_{\kappa}^{\prime}\right)^{1}\right) .
$$

Next choose a 1 -parameter family $f_{n}^{\rho}, \rho \in[0,1]$, where $f_{n}^{0}=f_{n}$ and $f_{n}^{1}=1$ and $f_{n}^{\rho}$ extends $f^{\rho}$. Using $f_{n}^{\rho} \alpha_{n}^{1}$, we obtain a continuation map

$$
\Theta_{n}^{\kappa}: \mathcal{A}\left(M_{n}, \alpha_{n}^{0}, J_{\kappa, n}^{0}\right) \rightarrow \mathcal{A}\left(M_{n}, \alpha_{n}^{1}, J_{\kappa, n}^{1}\right) .
$$


Let $K>0$. Then there exists $K^{\prime}>0$ such that

$$
\Theta_{\kappa}^{\prime}\left(\mathcal{A}_{\leq K}\left(M^{\prime},\left(\alpha^{\prime}\right)^{0},\left(J_{\kappa}^{\prime}\right)^{0}\right)\right) \subset \mathcal{A}_{\leq K^{\prime}}\left(M^{\prime},\left(\alpha^{\prime}\right)^{1},\left(J_{\kappa}^{\prime}\right)^{1}\right) .
$$

For sufficiently large $\kappa$, there exists $n(\kappa)$ such that if $n \geq n(\kappa)$ then the following diagram is commutative:

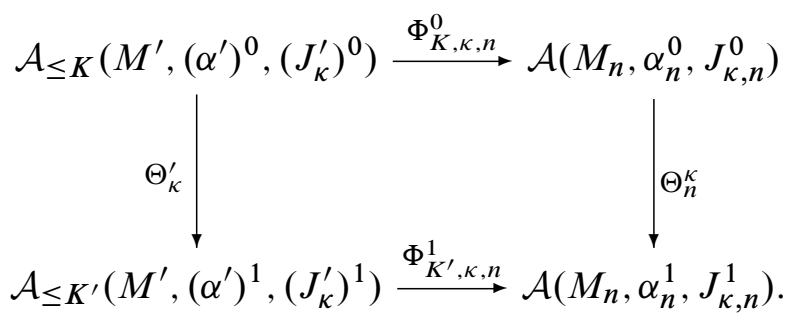

The proof follows from combining Step 1 of Section 6.2 and Theorem 10.1.

Given $\kappa^{\prime}>\kappa>0$ and $n^{\prime}>n>0$, let

$$
\left(\Psi_{n, n^{\prime}}^{\kappa, \kappa^{\prime}}\right)^{0}=j_{\kappa, \kappa^{\prime}}^{0} \circ \Psi_{n, n^{\prime}}^{0}: \mathcal{A}\left(M_{n}, \alpha_{n}^{0}, J_{\kappa, n}^{0}\right) \rightarrow \mathcal{A}\left(M_{n^{\prime}}, \alpha_{n^{\prime}}^{0}, J_{\kappa^{\prime}, n^{\prime}}^{0}\right)
$$

be the continuation map from last section; similarly define $\left(\Psi_{n, n^{\prime}}^{\kappa, \kappa^{\prime}}\right)^{1}$.

In order to take direct limits, we need to verify that the following diagrams

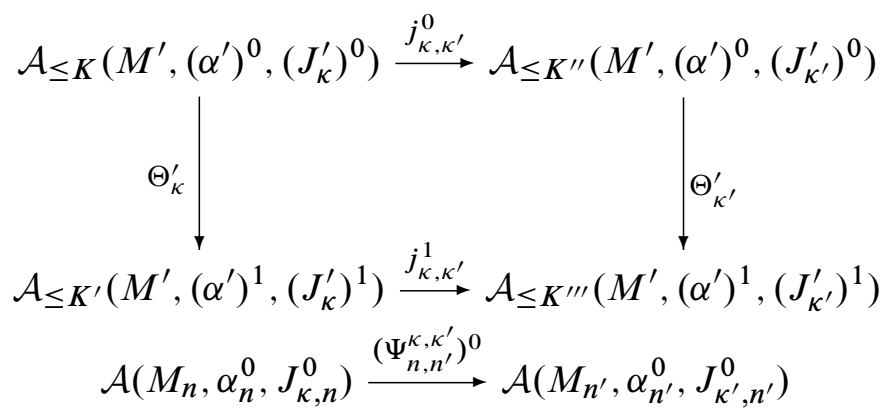

(40)

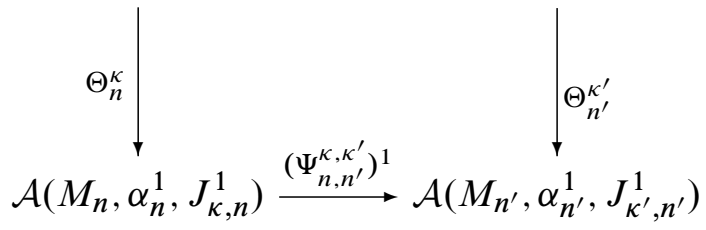

commute up to chain homotopy. This follows from the fact that, in either case, the symplectic cobordisms corresponding to the compositions (together with their almost complex structures) are homotopic. Taking direct limits, we obtain Diagram (37). 


\subsection{The ECH case}

In this section we explain how to prove Theorem 1.9(2), assuming the existence of appropriate cobordism maps on sutured $\mathrm{ECH}$, analogous to the cobordism maps on $\mathrm{ECH}$ of closed contact 3-manifolds defined in [27].

First observe that the ECH setup is much simpler since we do not need to use the parameter $\kappa$. Let $C\left(M^{\prime}, \alpha^{\prime}, J^{\prime}\right)$ be the $\mathrm{ECH}$ chain complex ( $\mathbb{Z}$-module) generated by the orbits sets of $R_{\alpha^{\prime}}$ and whose boundary map counts $J^{\prime}$-holomorphic curves. Also let $C_{0}\left(M_{n}, \alpha_{n}, J_{n}\right)$ be the subcomplex of the ECH chain complex $C\left(M_{n}, \alpha_{n}, J_{n}\right)$ which counts orbit sets which have zero intersection with $S_{\infty}$. As before, $C\left(M^{\prime}, \alpha^{\prime}, J^{\prime}\right)$ and the subcomplexes $C_{0}\left(M_{n}, \alpha_{n}, J_{n}\right)$ for different $n$ are all isomorphic as $\mathbb{Z}$-modules, although not necessarily as chain complexes.

Fix $n>0$. By analogy with [27], it is conjectured that given $K>0$, for sufficiently large $K^{\prime}$, the cobordism in Lemma 10.5 induces a chain map

$$
\Psi_{n, n+1}^{K, K^{\prime}}: C_{\leq K}\left(M_{n}, \alpha_{n}, J_{n}\right) \rightarrow C_{\leq K^{\prime}}\left(M_{n+1}, \alpha_{n+1}, J_{n+1}\right),
$$

which depends on some choices, but which has the following two properties: First, $\Psi_{n, n+1}^{K, K^{\prime}}$ is given by some unspecified count of (possibly broken) holomorphic curves between orbit sets $\vec{\gamma}$ for $\left(M_{n}, \alpha_{n}\right)$ and $\vec{\gamma}^{\prime}$ for $\left(M_{n+1}, \alpha_{n+1}\right)$, in the cobordism $\left(\mathbb{R} \times M_{n}^{*}, J\right)$ given in the proof of Lemma 10.5. Second, on the subset $\mathbb{R} \times M^{\prime}$ where the almost complex structure is cylindrical, trivial holomorphic cylinders over closed Reeb orbits are always counted in $\Psi_{n, n+1}^{K, K^{\prime}}$.

We now claim that the following commutative diagram of chain complexes exists:

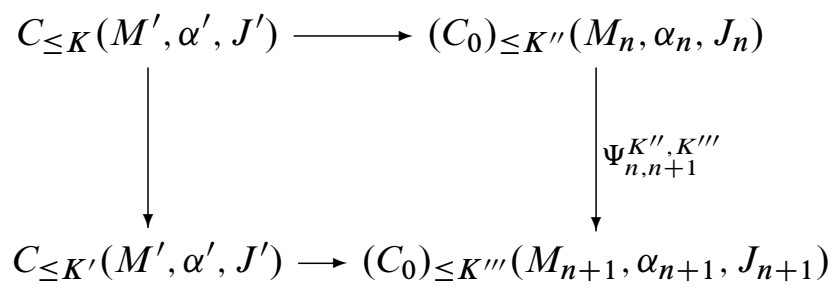

Here we are given $K^{\prime}>K>0$; we choose $n=n(K)>0, K^{\prime \prime} \geq K$ and $K^{\prime \prime \prime}=$ $K^{\prime \prime \prime}\left(n, K^{\prime \prime}\right) \geq K^{\prime}$. First note that $\Psi_{n, n+1}^{K^{\prime \prime}, K^{\prime \prime \prime}}$ is given by some count of holomorphic curves in the cobordism. On the other hand, Theorem 10.4 shows that, if $\vec{\gamma}$ is a generator of $\left(C_{0}\right)_{\leq K^{\prime \prime}}\left(M_{n}, \alpha_{n}, J_{n}\right)$ which comes from $C_{\leq K}\left(M^{\prime}, \alpha^{\prime}, J^{\prime}\right)$, then no holomorphic subvariety in $\left(\mathbb{R} \times M_{n}^{*}, J\right)$ which flows from $\vec{\gamma}$ can cross the "neck region", ie cross $S_{\infty}$, provided $n$ is chosen to be sufficiently large. Furthermore, once we know that no curve from $\vec{\gamma}$ crosses the "neck region", we are now in the symplectization portion, and we only have trivial cylinders. Hence $\Psi_{n, n+1}^{K^{\prime \prime}, K^{\prime \prime \prime}}$ 
maps $\vec{\gamma} \mapsto \vec{\gamma}$ if $\vec{\gamma}$ comes from $C_{\leq K}\left(M^{\prime}, \alpha^{\prime}, J^{\prime}\right)$. This proves the commutativity of Diagram (41).

For other $\vec{\gamma}$ in $\left(C_{0}\right)_{\leq K^{\prime \prime}}\left(M_{n}, \alpha_{n}, J_{n}\right)$, considerations of $\omega$ in Lemma 10.5, together with the fact that $\Psi_{n, n+1}^{K^{\prime \prime}, K^{\prime \prime \prime}}$ is some count of holomorphic curves, proves that $\Psi_{n, n+1}^{K^{\prime \prime}, K^{\prime \prime \prime}}$ maps $\vec{\gamma}$ to $\vec{\gamma}$ plus terms with lower action. (Note that $\omega$ is not the exact symplectic form which gives the exact symplectic cobordism, but is just some taming form for $J$.)

Arguing as in the contact homology case, we obtain an inclusion

$$
\lim _{K \rightarrow \infty} E C H_{\leq K}\left(M^{\prime}, \alpha^{\prime}, J^{\prime}\right) \hookrightarrow \lim _{n \rightarrow \infty}\left(E C H_{0}\right)_{\leq K^{\prime \prime}(n)}\left(M_{n}, \alpha_{n}, J_{n}\right),
$$

where $E C H_{0}$ is the homology for $C_{0}$. More precisely, the limit on the right-hand side is over $n \rightarrow \infty$ and $K^{\prime \prime}(n)$ is a sequence $\rightarrow \infty$ which depends on both $n$ and $K^{\prime \prime}(n-1)$. The left-hand side is $E C H\left(M^{\prime}, \Gamma^{\prime}, \xi^{\prime}\right)$, and the right-hand side equals $E C H_{0}(M, \Gamma, \xi)$, under our conjecture that $\operatorname{ECH}(M, \Gamma, \xi)$ does not depend on the choice of contact form or almost complex structure.

\section{Gluing along a convex submanifold}

Let $(M, \Gamma, \xi)$ be a contact manifold with convex boundary and let $S \subset M$ be a closed convex submanifold with dividing set $\Gamma_{S}$. Also let $\left(M^{\prime}, \Gamma^{\prime}, \xi^{\prime}\right)$ be the sutured contact manifold obtained by splitting $M$ along $S$ and applying Lemma 4.1.

The goal of this section is to prove the following:

Theorem 11.1 There is a canonical map

$$
\Phi: H C\left(M^{\prime}, \Gamma^{\prime}, \xi^{\prime}\right) \rightarrow H C(M, \Gamma, \xi) .
$$

In this section we will treat the case of contact homology; the proofs for embedded contact homology are similar.

According to Lemma 4.10, there is a contact 1 -form $\alpha^{\prime}$ which is adapted to the sutured contact manifold $\left(M^{\prime}, \Gamma^{\prime}, U\left(\Gamma^{\prime}\right), \xi^{\prime}\right)$ and an extension to $\left(M_{n}, \alpha_{n, g_{0}, g_{1}}\right)$ which is contactomorphic to $(M, \Gamma, U(\Gamma), \xi)$. Here $n>0$ and $g_{0}, g_{1}$ are functions depending on $n$. In this section we assume that $V \times D^{2}$ is the union of all the fillings of $M_{n}^{\prime}$, unlike in Section 4.4 where it was assumed to be just one connected component. It is clear that there is an inclusion

$$
\Phi: \mathcal{A}\left(M^{\prime}, \Gamma^{\prime}, \alpha^{\prime}, J^{\prime}\right) \rightarrow \mathcal{A}\left(M_{n}, \alpha_{n, g_{0}, g_{1}}, J_{n, g_{0}, g_{1}}\right) ;
$$

we would like to prove that $\Phi$ is a chain map. 
Our first task is to prove that, given $K>0$, for sufficiently large $n$ there exist $g_{0}, g_{1}$ so that the inclusion

$$
\Phi_{K}: \mathcal{A}_{\leq K}\left(M^{\prime}, \alpha^{\prime}, J^{\prime}\right) \rightarrow \mathcal{A}\left(M_{n}, \alpha_{n, g_{0}, g_{1}}, J_{n, g_{0}, g_{1}}\right)
$$

is a chain map, ie $\Phi_{K} \circ \partial^{\prime}=\partial \circ \Phi_{K}$, where $\partial$ and $\partial^{\prime}$ are boundary maps for $M_{n}$ and $M^{\prime}$. For this, it suffices to show the following:

Lemma 11.2 Suppose the orbits of $\gamma$ are contained in $\left(M^{\prime}, \alpha^{\prime}\right)$. Then for sufficiently large $n>0$ there exist $g_{0}, g_{1}$ (depending on $n$ ) such that

$$
\mathcal{M}_{g}\left(\underline{\gamma} ; \underline{\gamma}^{\prime} ; \mathbb{R} \times M_{n}^{*}, J_{n, g_{0}, g_{1}}\right)=\mathcal{M}_{g}\left(\underline{\gamma} ; \underline{\gamma^{\prime}} ; \mathbb{R} \times\left(M^{\prime}\right)^{*}, J^{\prime}\right),
$$

if the orbits of $\underline{\gamma}^{\prime}$ are contained in $\left(M^{\prime}, \alpha^{\prime}\right)$, and

$$
\mathcal{M}_{g}\left(\underline{\gamma} ; \underline{\gamma^{\prime}} ; \mathbb{R} \times M_{n}^{*}, J_{n, g_{0}, g_{1}}\right)=\varnothing
$$

otherwise.

Proof Let $F=(a, f):(\Sigma, j, \mathbf{m}) \rightarrow\left(\mathbb{R} \times M_{n}^{*}, J_{n, g_{0}, g_{1}}\right)$ be an element of the moduli space $\mathcal{M}_{g}\left(\underline{\gamma} ; \underline{\gamma^{\prime}} ; \mathbb{R} \times M_{n}^{*}, J_{n, g_{0}, g_{1}}\right)$. It suffices to show the following:

(1) There is no $F$ from $\underline{\gamma}$ to $\underline{\gamma}^{\prime}$, where some component of $\underline{\gamma}^{\prime}$ is not strictly contained in $M^{\prime}$.

(2) No $F$ from $\underline{\gamma}$ to $\underline{\gamma}^{\prime}$ with all components of $\underline{\gamma}^{\prime}$ in $M^{\prime}$ has $\operatorname{Im}(f)$ which nontrivially intersects $V \times D^{2}, S_{1}^{+} \times\left\{\frac{n}{2}\right\}$ or $S_{1}^{-} \times\left\{\frac{n}{2}\right\}$.

(1) is easy since we can choose $n, g_{0}, g_{1}$ so that all the closed orbits in $\left(M_{n}, \alpha_{n, g_{0}, g_{1}}\right)$ which are not in $\left(M^{\prime}, \alpha^{\prime}\right)$ have arbitrarily large action; see Lemma 4.10.

We now argue (2). First take $n$ sufficiently large so any $F \in \mathcal{M}_{g}\left(\underline{\gamma} ; \underline{\gamma^{\prime}} ; \mathbb{R} \times M_{n}^{*}, J_{n, g_{0}, g_{1}}\right)$ with image inside $\mathbb{R} \times\left(M_{n}^{\prime}\right)^{*}$ has image inside $\mathbb{R} \times\left(M^{\prime}\right)^{*}$. This can be done by Lemma 8.2. In addition to $n$, the functions $g_{0}, g_{1}$ will depend on the choice of $B>0$. In particular, we take $B$ so that $\operatorname{Im}\left(g_{0}, g_{1}\right)$ contains the line segment between $(a, 1)$ and $(a, B)$. Let $U_{B} \subset V \times D^{2}$ be the subset consisting of points $(x, r, \theta)$, where $\left(g_{0}(r), g_{1}(r)\right)$ is contained in this line segment. Also let $\beta_{0}^{\prime}$ be the restriction of $\alpha^{\prime}$ to $\partial R_{+}\left(\Gamma^{\prime}\right)$. On $U_{B}, \alpha_{n, B}=\alpha_{n, g_{0}(B), g_{1}(B)}$ is of the form $a d \theta+\beta_{B}$, where $\beta_{B}$ is a symplectization of $\beta_{0}^{\prime}$ in the $-r$-direction. Alternatively, we write $M_{n, B}^{\prime \prime}=M_{n}^{\prime} \cup U_{B}$ and use coordinates $(t, \tau, x)$ on

$$
U_{B} \simeq(\mathbb{R} / a \mathbb{Z}) \times\left[0, \tau_{B}\right] \times V
$$

so that $\alpha_{n, B}=d t+e^{\tau} \beta_{0}^{\prime}(x)$. Let $\hat{S}_{i}^{+}$be the extension of $S_{i}^{+}$to $M_{n, B}^{\prime \prime}$ so that $\partial \hat{S}_{i}^{+} \subset \partial M_{n, B}^{\prime \prime}$. Let $\left(J_{B}\right)_{0}$ be an almost complex structure on $\hat{S}_{i}^{+}$which is adapted to 
the symplectization $d\left(e^{\tau} \beta_{0}^{\prime}(x)\right)$, and let $J_{n, B}$ be a tailored almost complex structure on $M_{n}^{*}$ whose projection to $\widehat{S}_{i}^{+}$equals $\left(J_{B}\right)_{0}$.

We claim that, for sufficiently large $B>0$, all holomorphic maps $F_{B}=\left(a_{B}, f_{B}\right) \in$ $\mathcal{M}_{g}\left(\underline{\gamma} ; \underline{\gamma^{\prime}} ; \mathbb{R} \times M_{n}^{*}, J_{n, B}\right)$ are disjoint from $\mathbb{R} \times U_{B}$. (Note that, by the strict plurisubharmonicity of $\tau, F_{n, B}$ is disjoint from $U_{B}$ if and only if $F_{n, B}$ is disjoint from $\partial M_{n, B}^{\prime \prime}$.) The argument is similar to that of Lemma 9.2, only easier. Arguing by contradiction, suppose there is a sequence $F_{B_{i}}=\left(a_{B_{i}}, f_{B_{i}}\right)$ where $f_{B_{i}}$ nontrivially intersects $U_{B_{i}}$ and $B_{i} \rightarrow \infty$. Writing $v_{B_{i}}$ as the projection of $f_{B_{i}}$ to $\left[0, \tau_{B_{i}}\right] \times V$ whenever applicable, in the limit as $B_{i} \rightarrow \infty$ we eventually obtain a finite energy cylinder $v_{\infty}:[0, \infty) \times S^{1} \rightarrow[0, \infty) \times V$. However, this contradicts the energy bound as follows: First, the $F_{B_{i}}$ have bounded $d \alpha_{n, B_{i}}$-energy since $\underline{\gamma}$ and $\underline{\gamma}^{\prime}$ are fixed. On $U_{B_{i}}$, $d \alpha_{n, B_{i}}=d\left(e^{\tau} \beta_{0}^{\prime}\right)$, and a cylinder over a Reeb orbit of $\beta_{0}^{\prime}$ has unbounded $d\left(e^{\tau} \beta_{0}^{\prime}\right)-$ area, a contradiction.

Once we know that $F_{B}$ is disjoint from $\mathbb{R} \times U_{B}$, by our choice of $n \gg 0, F_{B}$ has image inside $\mathbb{R} \times\left(M^{\prime}\right)^{*}$ by Lemma 8.2. This concludes the proof of Lemma 11.2.

Case of dimension three We give an alternate, more straightforward proof of Lemma 11.2 when $\operatorname{dim} M=3$.

Lemma 11.3 Let $F=(a, f):(\Sigma, j, \mathbf{m}) \rightarrow\left(\mathbb{R} \times M_{n}^{*}, J_{n, g_{0}, g_{1}}\right)$ be a holomorphic map which is asymptotic to $\underline{\gamma}$ at $s \rightarrow+\infty$ and asymptotic to $\underline{\gamma}^{\prime}$ at $s \rightarrow-\infty$. If $\operatorname{dim} M=3$ and the orbits of $\underline{\bar{\gamma}}$ and $\underline{\gamma}^{\prime}$ lie in $M^{\prime}$, then the image of $f$ is disjoint from $V \times\{0\}$ if $n$ is sufficiently large.

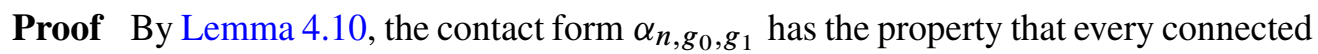
component of $V \times\{0\}$ is a periodic orbit of the Reeb flow. Hence all intersection points between $V \times\{0\}$ and $C=\operatorname{Im}(f)$ are positive, by the positivity of intersections in dimension four. Observe that $V \times\{0\}$ is the oriented boundary of a surface $S$ which is an extension of $R_{+}\left(\Gamma^{\prime}\right) \subset M^{\prime}$ to $M_{n}$, and $R_{+}\left(\Gamma^{\prime}\right)$ is disjoint from $\underline{\gamma} \cup \underline{\gamma^{\prime}}$. We may assume without loss of generality that $C \pitchfork S$. If $C$ has nontrivial intersection with $\partial S$, then there is a properly embedded $\operatorname{arc} c$ on $S$ which connects from $\partial S$ to itself. However, $C$ and $V \times\{0\}=\partial S$ intersect positively at one endpoint of $c$ and negatively at the other endpoint, a contradiction. We conclude that the image of $f$ is disjoint from $V \times\{0\}$.

We claim that $C=\operatorname{Im}(f)$ is contained in $\left(M_{n}^{\prime}\right)^{*}$. Assume for convenience that $V$ is connected. By Lemma 11.3, $C$ is disjoint from $V \times\{0\}$. Let $T_{r=1}$ be the torus $\{r=1\} \subset V \times D^{2}$. It then follows that $C \cap T_{r=1}$ is homologous to $\varnothing$ on $T_{r=1}$. On the 
other hand, on $T_{r=1}$ the Reeb vector field is parallel to $\partial_{\theta}$ and $C$ must be positively transverse to $\partial_{\theta}$ by intersection positivity. (By a slight perturbation if necessary, we may assume that $C \cap T_{r=1}$ is an immersion.) If we take an oriented identification $T_{r=1}=\mathbb{R}^{2} / \mathbb{Z}^{2}$ with orientation on $T_{r=1}$ equal to the boundary orientation of $V \times D^{2}$ and choose coordinates $(\theta /(2 \pi), x)$, and we set $\Sigma^{\prime}=\Sigma-f^{-1}\left(V \times D^{2}\right)$, then $d x$ is everywhere positive on $\left.f\right|_{\partial \Sigma^{\prime}}$. Since $\left.f\right|_{\partial \Sigma^{\prime}}$ is not homologically zero if $C$ intersects $T_{r=1}$, we conclude that $C$ does not enter $V \times D^{2}$. Now we can apply the argument in Lemma 8.2 to show that, for sufficiently large $n$, no $f$ intersects $S_{1}^{+} \times\left\{\frac{n}{2}\right\}$ and $S_{1}^{-} \times\left\{\frac{n}{2}\right\}$ as described in Section 4.4. Hence we can view $F$ as sitting inside $\mathbb{R} \times\left(M^{\prime}\right)^{*}$.

Returning to the proof of Theorem 11.1, we now define two chain maps $\Psi_{B, B+1}^{n}$ and $\Psi_{B}^{n, n+1}$, where $n$ and $B$ are positive integers:

The first chain map Given contact forms $\alpha_{n, B}$ and $\alpha_{n, B+1}$ on $M_{n}$, arrange them via an isotopy so that the forms agree on $M_{n, B}^{\prime \prime}$ and the contact structures agree on $M_{n}-M_{n, B}^{\prime \prime}$. We also assume that $J_{n, B}$ and $J_{n, B+1}$ agree on $M_{n, B}^{\prime \prime}$, and are induced by $J^{\prime}$ on $M^{\prime}$. Then interpolating between $\alpha_{n, B}$ and $\alpha_{n, B+1}$ and between the almost complex structures gives us a symplectic cobordism and a corresponding chain map

$$
\Psi_{B, B+1}^{n}: \mathcal{A}\left(M_{n}, \alpha_{n, B}, J_{n, B}\right) \rightarrow \mathcal{A}\left(M_{n}, \alpha_{n, B+1}, J_{n, B+1}\right) .
$$

An argument identical to that of Lemma 11.2 shows that, given $K>0$, for sufficiently large $n$ there exists $B_{0}(n)$ such that for $B \geq B_{0}(n)$ the following diagram commutes:

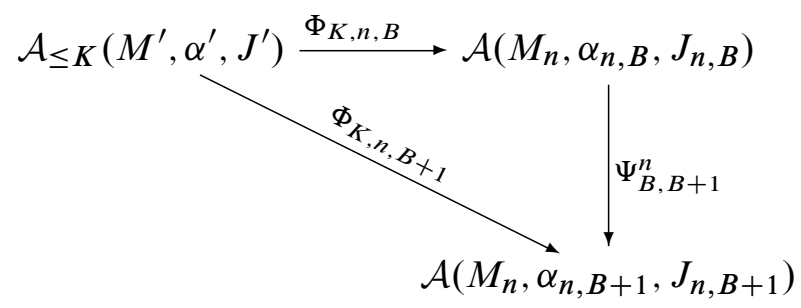

In particular, if $\gamma \in \mathcal{A}_{\leq K}\left(M^{\prime}, \alpha^{\prime}, J^{\prime}\right)$, then $\Psi_{B, B+1}^{n} \circ \Phi_{K, n, B}(\gamma)=\Phi_{K, n, B+1}(\gamma)$.

The second chain map Given $\alpha_{n, B}$ on $M_{n}$ and $\alpha_{n+1, B}$ on $M_{n+1}$, we take a diffeomorphism $i: M_{n} \stackrel{\sim}{\rightarrow} M_{n+1}$ which is similar to the one defined in the paragraph before Lemma 8.3: it takes $M^{\prime}$ to $M^{\prime}$ by the identity and sends $M_{n, B}^{\prime \prime} \stackrel{\sim}{\rightarrow} M_{n+1, B}^{\prime \prime}$, while stretching $M_{n, B}^{\prime \prime}-M^{\prime}$ in the $\partial_{t}$-direction (ie the Reeb direction) so that $i^{*}\left(d t+\beta^{\prime}\right)=d f+\beta^{\prime}$ and $|\partial f / \partial t-1|=O(1 / n)$. Also assume that $J_{n, B}$ and $J_{n+1, B}$ agree with $J^{\prime}$ on $M^{\prime}$ and project to the same almost complex structure on $\hat{S}_{i}^{+}$. Interpolating between $\alpha_{n, B}$ and $i^{*} \alpha_{n+1, B}$, we obtain

$$
\Psi_{B}^{n, n+1}: \mathcal{A}\left(M_{n}, \alpha_{n, B}, J_{n, B}\right) \rightarrow \mathcal{A}\left(M_{n+1}, \alpha_{n+1, B}, J_{n+1, B}\right) .
$$


Given $K>0$, for sufficiently large $n$ there exists $B_{0}(n)$ such that for $B \geq B_{0}(n)$ the following diagram commutes:

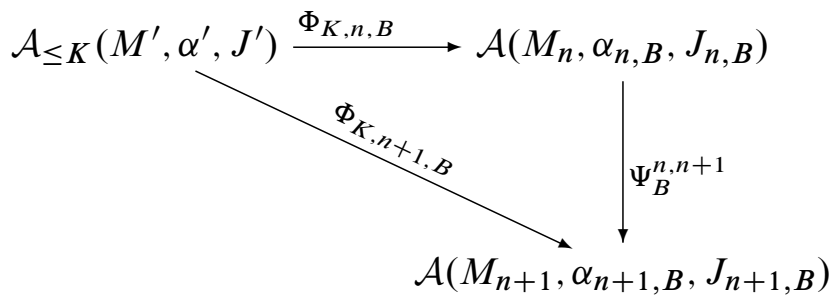

Moreover, if $\gamma \in \mathcal{A}_{\leq K}\left(M^{\prime}, \alpha^{\prime}, J^{\prime}\right)$, then $\Psi_{B}^{n, n+1} \circ \Phi_{K, n, B}(\gamma)=\Phi_{K, n+1, B}(\gamma)$. First we pick $n$ so that any $F \in \mathcal{M}_{g}\left(\underline{\gamma} ; \underline{\gamma^{\prime}} ; \mathbb{R} \times M_{n}^{*}, J_{n, B}\right)$ with image inside $\mathbb{R} \times\left(M_{n}^{\prime}\right)^{*}$ has image inside $\mathbb{R} \times\left(M^{\prime}\right)^{*}$, as in Lemma 8.3. Next, we pick $B_{0}(n)$ to bound the $\tau$-direction as in the proof of Lemma 11.2.

Definition of the map $\Phi$ By repeatedly composing the maps of type $\Psi_{B, B+1}^{n}$ and $\Psi_{B}^{n, n+1}$, we obtain the chain map

$$
\Psi_{B, B^{\prime}}^{n, n^{\prime}}=\Psi_{B^{\prime}}^{n^{\prime}-1, n^{\prime}} \circ \cdots \circ \Psi_{B^{\prime}}^{n, n+1} \circ \Psi_{B^{\prime}-1, B^{\prime}}^{n} \circ \cdots \circ \Psi_{B, B+1}^{n} .
$$

Here $i_{K, K^{\prime}}$ is the natural inclusion. Given $K^{\prime}>K>0$, there exist $n^{\prime}>n>0$ and $B^{\prime}=B^{\prime}\left(n^{\prime}\right)>B=B(n)>0$ so that $\Phi_{K, n, B}$ and $\Phi_{K^{\prime}, n^{\prime}, B^{\prime}}$ both map $\gamma \mapsto \gamma$ and the following diagram commutes:

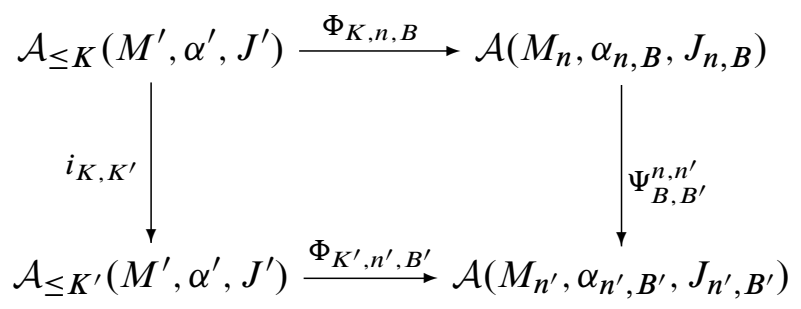

Taking direct limits, we have

$$
\Phi: \lim _{K \rightarrow \infty} H C_{\leq K}\left(M^{\prime}, \alpha^{\prime}, J^{\prime}\right) \rightarrow \lim _{n \rightarrow \infty} H C\left(M_{n}, \alpha_{n, B(n)}, J_{n, B(n)}\right) .
$$

Since the $H C\left(M^{\prime}, \alpha^{\prime}\right)=\lim _{K \rightarrow \infty} H C_{\leq K}\left(M^{\prime}, \alpha^{\prime}\right)$ and the maps $\Psi_{B, B^{\prime}}^{n, n^{\prime}}$ are always isomorphisms, we have defined the map $\Phi$ in Theorem 11.1.

Proof that $\Phi$ is independent of choices Let $\left(\alpha^{\prime}\right)^{i}, i=0,1$, be two contact forms which are adapted to $\left(M^{\prime}, \Gamma^{\prime}, U\left(\Gamma^{\prime}\right), \xi^{\prime}\right)$ and let $\left(J^{\prime}\right)^{i}$ be almost complex structures tailored to $\left(\alpha^{\prime}\right)^{i}$. Also let $\left(M_{n}, \alpha_{n, B}^{i}, J_{n, B}^{i}\right)$ be the extensions of $\left(M^{\prime},\left(\alpha^{\prime}\right)^{i},\left(J^{\prime}\right)^{i}\right)$, as described earlier. Let $\left(\beta^{\prime}\right)_{0}^{i}=\left.\left(\alpha^{\prime}\right)^{i}\right|_{\partial R_{+}\left(\Gamma^{\prime}\right)}$ and $\left(\beta^{\prime}\right)^{i}=\left.\left(\alpha^{\prime}\right)^{i}\right|_{R_{+}\left(\Gamma^{\prime}\right)}$. As in the 
proof of Proposition 10.7, we can write $\left(\alpha^{\prime}\right)^{0}=f\left(\alpha^{\prime}\right)^{1}$ and $\left(\beta^{\prime}\right)_{0}^{0}=\left(\beta^{\prime}\right)_{0}^{1}$. Also, if the manifold $M_{n}$ is fixed, then we can write $\alpha_{n}^{0}=f_{n, B} \alpha_{n, B}^{1}$.

We construct a 1 -parameter family $f(\rho)\left(\alpha^{\prime}\right)^{1}, \rho \in[0,1], f(0)=f, f(1)=1$, to construct a symplectic cobordism and a continuation map

$$
\Theta^{\prime}: \mathcal{A}\left(M^{\prime},\left(\alpha^{\prime}\right)^{0},\left(J^{\prime}\right)^{0}\right) \rightarrow \mathcal{A}\left(M^{\prime},\left(\alpha^{\prime}\right)^{1},\left(J^{\prime}\right)^{1}\right) .
$$

Next we extend $f(\rho)$ to $f_{n, B}(\rho)$, so that $f_{n, B}(0)=f_{n, B}$ and $f_{n, B}(1)=1$. Using $f_{n, B}(\rho) \alpha_{n, B}^{1}$, we obtain a continuation map

$$
\Theta_{B}^{n}: \mathcal{A}\left(M_{n}, \alpha_{n, B}^{0}, J_{n, B}^{0}\right) \rightarrow \mathcal{A}\left(M_{n}, \alpha_{n, B}^{1}, J_{n, B}^{1}\right) .
$$

Let $K>0$. Then there exists $K^{\prime}>0$ such that

$$
\Theta^{\prime}\left(\mathcal{A}_{\leq K}\left(M^{\prime},\left(\alpha^{\prime}\right)^{0},\left(J^{\prime}\right)^{0}\right)\right) \subset \mathcal{A}_{\leq K^{\prime}}\left(M^{\prime},\left(\alpha^{\prime}\right)^{1},\left(J^{\prime}\right)^{1}\right) .
$$

For sufficiently large $n$ there exists $B_{0}(n)$ such that for $B \geq B_{0}(n)$ the following diagram commutes:

$$
\begin{gathered}
\mathcal{A}_{\leq K}\left(M^{\prime},\left(\alpha^{\prime}\right)^{0},\left(J^{\prime}\right)^{0}\right) \stackrel{\Phi_{K, n, B}^{0}}{\longrightarrow} \mathcal{A}\left(M_{n}, \alpha_{n, B}^{0}, J_{n, B}^{0}\right) \\
\mathcal{\Theta}_{\leq K^{\prime}}\left(M^{\prime},\left(\alpha^{\prime}\right)^{1},\left(J^{\prime}\right)^{1}\right) \stackrel{\Phi_{K^{\prime}, n, B}^{1}}{\longrightarrow} \mathcal{A}\left(M_{n}, \alpha_{n, B}^{1}, J_{n, B}^{1}\right)
\end{gathered}
$$

Taking direct limits, we obtain the commutative diagram

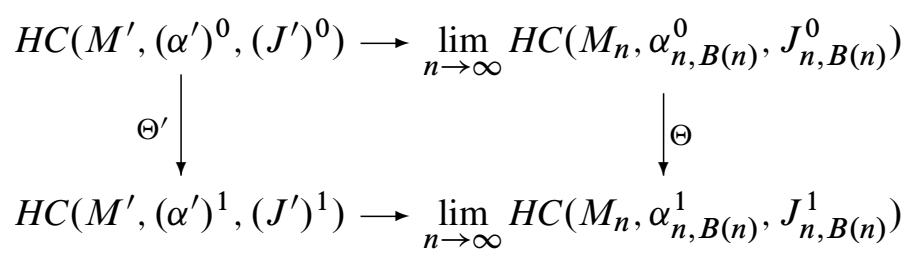

which proves that the two versions of the map $\Phi$ agree.

Acknowledgements It is a pleasure to thank Yasha Eliashberg and Jian He for very helpful discussions. Part of this work was done while KH, MH and PG visited MSRI during the academic year 2009-2010; they would like to thank MSRI and the organizers of the "Symplectic and Contact Geometry and Topology" and the "Homology Theories of Knots and Links" programs for their hospitality. We thank the anonymous referee for many detailed comments.

VC was supported by the Institut Universitaire de France, ANR Symplexe and ANR Floer Power. PG was supported by ANR Floer Power. KH was supported by NSF Grant DMS-0805352. MH was supported by NSF Grant DMS-0806037. 


\section{References}

[1] F Bourgeois, V Colin, Homologie de contact des variétés toroüdales, Geom. Topol. 9 (2005) 299-313 MR2116317

[2] F Bourgeois, T Ekholm, Y Eliashberg, Effect of Legendrian surgery arXiv: 0911.0026

[3] F Bourgeois, Y Eliashberg, H Hofer, K Wysocki, E Zehnder, Compactness results in symplectic field theory, Geom. Topol. 7 (2003) 799-888 MR2026549

[4] F Bourgeois, K Mohnke, Coherent orientations in symplectic field theory, Math. Z. 248 (2004) 123-146 MR2092725

[5] Y Chekanov, Differential algebra of Legendrian links, Invent. Math. 150 (2002) 441483 MR1946550

[6] K Cieliebak, K Mohnke, Compactness for punctured holomorphic curves, J. Symplectic Geom. 3 (2005) 589-654 MR2235856 Conference on Symplectic Topology

[7] V Colin, P Ghiggini, K Honda, M Hutchings, Sutures and contact homology II, in preparation

[8] V Colin, K Honda, Constructions contrôlées de champs de Reeb et applications, Geom. Topol. 9 (2005) 2193-2226 MR2209370

[9] D L Dragnev, Fredholm theory and transversality for noncompact pseudoholomorphic maps in symplectizations, Comm. Pure Appl. Math. 57 (2004) 726-763 MR2038115

[10] T Ekholm, J Etnyre, M Sullivan, The contact homology of Legendrian submanifolds in $\mathbb{R}^{2 n+1}$, J. Differential Geom. 71 (2005) 177-305 MR2197142

[11] Y Eliashberg, A Givental, H Hofer, Introduction to symplectic field theory, from: “GAFA 2000 (Tel Aviv, 1999)", Geom. Funct. Anal. Special Volume, Part II (2000) 560-673 MR1826267

[12] Y Eliashberg, M Gromov, Convex symplectic manifolds, from: "Several complex variables and complex geometry, Part 2 (Santa Cruz, CA, 1989)", (E Bedford, J P D’Angelo, R E Greene, S G Krantz, editors), Proc. Sympos. Pure Math. 52, Amer. Math. Soc. (1991) 135-162 MR1128541

[13] O Fabert, Obstruction bundles over moduli spaces with boundary and the action filtration in symplectic field theory arXiv:0709.3312

[14] D Gabai, Foliations and the topology of 3-manifolds, J. Differential Geom. 18 (1983) 445-503 MR723813

[15] E Giroux, Convexité en topologie de contact, Comment. Math. Helv. 66 (1991) 637-677 MR1129802

[16] E Giroux, Géométrie de contact: de la dimension trois vers les dimensions supérieures, from: "Proceedings of the International Congress of Mathematicians, Vol. II (Beijing, 2002)", (T Li, editor), Higher Ed. Press, Beijing (2002) 405-414 MR1957051 
[17] R Golovko, The embedded contact homology of sutured solid tori II, in preparation

[18] R Golovko, The embedded contact homology of sutured solid tori, Algebr. Geom. Topol. 11 (2011) 1001-1031 MR2782551

[19] H Hofer, Pseudoholomorphic curves in symplectizations with applications to the Weinstein conjecture in dimension three, Invent. Math. 114 (1993) 515-563 MR1244912

[20] H Hofer, A general Fredholm theory and applications, from: "Current developments in mathematics, 2004”, (D Jerison, B Mazur, T Mrowka, W Schmid, R Stanley, S-T Yau, editors), Int. Press, Somerville, MA (2006) 1-71 MR2459290

[21] K Honda, W H Kazez, G Matić, Convex decomposition theory, Int. Math. Res. Not. (2002) 55-88 MR1874319

[22] K Honda, W H Kazez, G Matić, Right-veering diffeomorphisms of compact surfaces with boundary II, Geom. Topol. 12 (2008) 2057-2094 MR2431016

[23] C Hummel, Gromov's compactness theorem for pseudo-holomorphic curves, Progress in Math. 151, Birkhäuser Verlag, Basel (1997) MR1451624

[24] M Hutchings, An index inequality for embedded pseudoholomorphic curves in symplectizations, J. Eur. Math. Soc. 4 (2002) 313-361 MR1941088

[25] M Hutchings, The embedded contact homology index revisited, from: "New perspectives and challenges in symplectic field theory", (M Abreu, F Lalonde, L Polterovich, editors), CRM Proc. Lecture Notes 49, Amer. Math. Soc. (2009) 263-297 MR2555941

[26] M Hutchings, M Sullivan, Rounding corners of polygons and the embedded contact homology of $T^{3}$, Geom. Topol. 10 (2006) 169-266 MR2207793

[27] M Hutchings, C H Taubes, Proof of the Arnold chord conjecture in three dimensions $I I$, in preparation

[28] M Hutchings, C H Taubes, Gluing pseudoholomorphic curves along branched covered cylinders I, J. Symplectic Geom. 5 (2007) 43-137 MR2371184

[29] M Hutchings, C H Taubes, Gluing pseudoholomorphic curves along branched covered cylinders II, J. Symplectic Geom. 7 (2009) 29-133 MR2491716

[30] A Juhász, Holomorphic discs and sutured manifolds, Algebr. Geom. Topol. 6 (2006) 1429-1457 MR2253454

[31] A Juhász, Floer homology and surface decompositions, Geom. Topol. 12 (2008) 299350 MR2390347

[32] P Kronheimer, T Mrowka, Monopoles and three-manifolds, New Math. Monogr. 10, Cambridge Univ. Press (2007) MR2388043

[33] P Kronheimer, T Mrowka, Knots, sutures, and excision, J. Differential Geom. 84 (2010) 301-364 MR2652464

[34] A Cannas da Silva, V Guillemin, C Woodward, On the unfolding of folded symplectic structures, Math. Res. Lett. 7 (2000) 35-53 MR1748286 
[35] C H Taubes, The structure of pseudo-holomorphic subvarieties for a degenerate almost complex structure and symplectic form on $S^{1} \times B^{3}$, Geom. Topol. 2 (1998) 221-332 MR1658028

[36] C H Taubes, The Seiberg-Witten equations and the Weinstein conjecture, Geom. Topol. 11 (2007) 2117-2202 MR2350473

[37] C H Taubes, Embedded contact homology and Seiberg-Witten Floer cohomology I-IV, Geom. Topol. 14 (2010) 2497-2960 MR2746723

[38] I Ustilovsky, Infinitely many contact structures on $S^{4 m+1}$, Internat. Math. Res. Notices (1999) 781-791 MR1704176

VC, PG: Laboratoire de Mathématiques Jean Leray, UMR6629 du CNRS, Université de Nantes 2 rue de la Houssinière, F-44322 Nantes, France

KH: Department of Mathematics, University of Southern California 3620 S Vermont Ave, Los Angeles CA 90089, USA

MH: Mathematics Department, University of California, Berkeley 970 Evans Hall, Berkeley CA 94720, USA

vincent.colin@univ-nantes.fr, paolo.ghiggini@univ-nantes.fr, khonda@usc.edu, hutching@math.berkeley.edu

http://www.math.sciences.univ-nantes.fr/ vcolin/, http://www . math.sciences. univ-nantes.fr/ ghiggini, http://www-bcf.usc.edu/ khonda/, http://math.berkeley.edu/ hutching

Proposed: Yasha Eliashberg

Seconded: Peter S Ozsváth, Leonid Polterovich
Received: 27 April 2010

Revised: 12 July 2011 